\title{
On the Detection of Exomoons in Photometric Time Series
}

\author{
Dissertation \\ zur Erlangung des mathematisch-naturwissenschaftlichen Doktorgrades \\ "Doctor rerum naturalium" \\ der Georg-August-Universität Göttingen \\ im Promotionsprogramm PROPHYS \\ der Georg-August University School of Science (GAUSS)
}

vorgelegt von

Kai Oliver Rodenbeck

aus Göttingen, Deutschland

Göttingen, 2019 
Betreuungsausschuss

Prof. Dr. Laurent Gizon

Max-Planck-Institut für Sonnensystemforschung, Göttingen, Deutschland und Institut für Astrophysik, Georg-August-Universität, Göttingen, Deutschland

Prof. Dr. Stefan Dreizler

Institut für Astrophysik, Georg-August-Universität, Göttingen, Deutschland

Dr. Warrick H. Ball

School of Physics and Astronomy, University of Birmingham, UK vormals Institut für Astrophysik, Georg-August-Universität, Göttingen, Deutschland

Mitglieder der Prüfungskommision

Referent: Prof. Dr. Laurent Gizon

Max-Planck-Institut für Sonnensystemforschung, Göttingen, Deutschland und Institut für Astrophysik, Georg-August-Universität, Göttingen, Deutschland

Korreferent: Prof. Dr. Stefan Dreizler

Institut für Astrophysik, Georg-August-Universität, Göttingen, Deutschland

Weitere Mitglieder der Prüfungskommission:

Prof. Dr. Ulrich Christensen

Max-Planck-Institut für Sonnensystemforschung, Göttingen, Deutschland

Dr.ir. Saskia Hekker

Max-Planck-Institut für Sonnensystemforschung, Göttingen, Deutschland

Dr. René Heller

Max-Planck-Institut für Sonnensystemforschung, Göttingen, Deutschland

Prof. Dr. Wolfram Kollatschny

Institut für Astrophysik, Georg-August-Universität, Göttingen, Deutschland

Tag der mündlichen Prüfung: 29.4.2019 


\section{Bibliografische Information der Deutschen Nationalbibliothek}

Die Deutsche Nationalbibliothek verzeichnet diese Publikation in der Deutschen Nationalbibliografie; detaillierte bibliografische Daten sind im Internet über http: //dnb . d-nb . de abrufbar.

ISBN 978-3-944072-69-2

uni-edition $\mathrm{GmbH}$

http://www.uni-edition.de

(C) Kai Oliver Rodenbeck

(c) (i) $\Theta($ This work is distributed under a

By Na No Creative Commons Attribution 4.0 License

Printed in Germany 
Cover figure: Model of the planet and moon transits for the three transits of Kepler1625-b. This figure is adapted from Fig. 4 in Rodenbeck et al. (2018) (Fig. 2.4 in this thesis). 


\section{Contents}

\begin{tabular}{ll}
\hline Summary & 7
\end{tabular}

$\begin{array}{lll}1 & \text { Introduction } & 13\end{array}$

1.1 Exoplanets . . . . . . . . . . . . . . . . . . . . . . . . . . . . . . . . . .

1.1 .1 Overview . . . . . . . . . . . . . . . . 13

1.1 .2 Detection Methods $\ldots \ldots \ldots$. . . . . . . . . . . . . . 14

1.1.2.1 The Transit Method . . . . . . . . . . . . . . . . . 14

1.1.2.2 The Radial Velocity Method . . . . . . . . . . . . . . 17

1.2 Exomoons . . . . . . . . . . . . . . . . . . . 18

1.2 .1 Moons in our Solar System . . . . . . . . . . . . . . . . . . . 18

1.2.2 Moons as Tracers of Formation History . . . . . . . . . . . . . . 19

1.2.3 Habitability of Moons . . . . . . . . . . . . . . . . . . . 19

1.2 .4 Detection and Characterization Methods . . . . . . . . . . . . 20

1.2 .5 Surveys and Searches so far . . . . . . . . . . . . . . . . 22

1.2.6 The Exomoon Candidate around Kepler-1625 b . . . . . . . . . . 23

1.3 Data Sources for Exoplanet and Exomoon Studies . . . . . . . . . . . . . 24

1.4 Content of this Thesis . . . . . . . . . . . . . . . . . . . . 24

$2 \quad$ Paper I: Revisiting the exomoon candidate signal around Kepler-1625 b 27

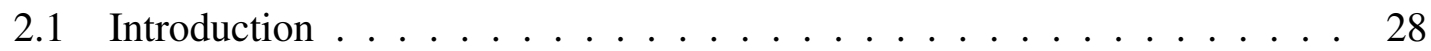

2.2 Methods . . . . . . . . . . . . . . . . . . . . 30

2.2 .1 Trigonometric detrending . . . . . . . . . . . . 31

2.2 .2 Transit model . . . . . . . . . . . . . . . . . . . . . . . 34

$2.2 .2 .1 \quad$ Planet-only model . . . . . . . . . . . . . . . . . . . . . . . . . 34

2.2 .2 .2 Planet-moon model . . . . . . . . . . . . . 35

2.2.2.3 Finding the posterior probability distribution . . . . . . 37

2.2 .3 Model selection . . . . . . . . . . . . . . . 38

2.2 .4 Injection-retrieval test $\ldots \ldots \ldots \ldots . \ldots . \ldots 38$

2.2.4.1 Transit injections into light curves . . . . . . . . . 39

2.2.4.2 Testing the model-selection algorithm on synthetic light curves with white noise only . . . . . . . . . . 39

2.2.4.3 Transit injection into real out-of-transit data . . . . . . 42

$2.2 .4 .4 \quad$ Detrending of the transit-injected light curves . . . . 42

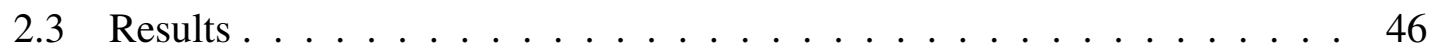

2.3.1 Analysis of the original Kepler-1625 b transits . . . . . . . . . . 46

2.3 .2 Injection-retrieval experiment $\ldots \ldots \ldots$. . . . . . . . 46 
2.4 Discussion . . . . . . . . . . . . . . . . . . . . . . . . . . . . 48

2.5 Conclusions . . . . . . . . . . . . . . . . . . . . . . . . . . 49

2.6 Acknowledgements . . . . . . . . . . . . . . . . 50

2.A $\quad$ Effect of the window length on the Bayesian information criterion . . . . 51

2.B Autocorrelation of detrended light curves . . . . . . . . . . . . 52

3 Paper II: An alternative interpretation of the exomoon candidate signal in the combined Kepler and Hubble data of Kepler-1625 53

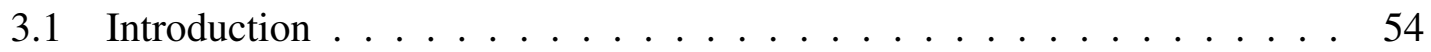

3.2 Methods . . . . . . . . . . . . . . . . . . 55

3.2 .1 Data preparation . . . . . . . . . . . . . 56

3.2.1.1 Extraction of the Hubble light curve . . . . . . . . . 56

3.2 .2 Proposed unseen planet . . . . . . . . . . . . . . 57

3.2.2.1 Mass-orbit constraints for a close-in planet . . . . . . . 57

3.2 .2 .2 Orbital stability $\ldots \ldots \ldots \ldots . \ldots 59$

3.3 Results . . . . . . . . . . . . . . . . . . 60

$3.3 .1 \quad$ PTMCMC sampling and $\triangle \mathrm{BIC} \ldots \ldots \ldots \ldots$

3.3 .2 Transit timing variations $\ldots \ldots \ldots 63$

3.4 Conclusions . . . . . . . . . . . . . . . . 65

4 Detection of Exomoons using Transit Variations 69

4.1 Observable Transit Variations . . . . . . . . . . . . . . . . . . . . . . . 69

4.2 Transit Variations for Different Moon Phases . . . . . . . . . . . . . 70

4.3 Transit Variations in Observed Transits . . . . . . . . . . . . . . . . . 72

5 Discussion 75

5.1 The Exomoon Candidate around Kepler-1625 b . . . . . . . . . . . 75

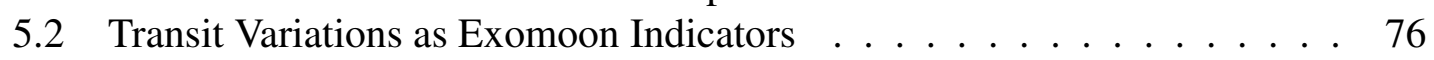

5.3 The Star-Planet-Moon Connection . . . . . . . . . . . . . . . . . . 77

5.4 Exomoon Detection using Machine Learning . . . . . . . . . . . . 78

5.5 Outlook . . . . . . . . . . . . . . . . . 80

\begin{tabular}{lr}
\hline Bibliography & 81
\end{tabular}

\begin{tabular}{ll}
\hline Publications & 91
\end{tabular}

\begin{tabular}{ll}
\hline Conferences & 91
\end{tabular}

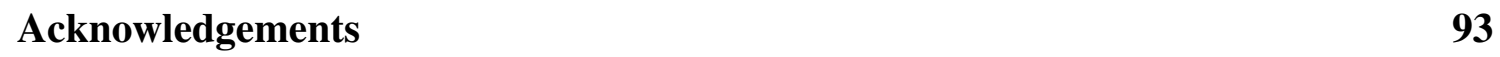

\begin{tabular}{ll}
\hline Curriculum vitae & 95
\end{tabular} 


\section{Summary}

One of the next big steps forwards for exoplanet science is the detection of moons around extrasolar planets. In this thesis I develop methods to detect and characterize these moons around extrasolar planets, with a focus on the moon candidate around the exoplanet Kepler-1625 b. The main part of this thesis is formed by two papers about the exomoon candidate around Kepler-1625 b and a short chapter about a new exomoon indicator we developed. The three topics and the main results are described shortly below.

In Chapter 2, we study the extrasolar planet Kepler-1625 b, for which the discovery of a moon candidate has been announced recently. We assess the reliability of this claim by performing an injection-retrieval experiment, where we inject model light curves with and without a moon signal into parts of the original Kepler-1625 light curve. We find that we can recover around 40 percent of the injected moons. However, we also find that we recover moons in around 10 percent of the light curves where no moon was injected. This is a high false-positive rate, considering that the survey that found the moon candidate looked at hundreds of stars. We also analyze the original light curve ourself, and find marginal evidence in favor of the existence of the moon.

In Chapter 3, we return to the moon candidate around Kepler-1625 b after an additional transit has been observed by the Hubble space telescope, and the moon parameters have been refined by the original discoverers. We perform an analysis of both the light curve detrended by the original discoverers of the moon candidate as well as our own detrending of the light curve. The analysis is improved compared to the first paper: The detrending of the light curve is done simultaneously to the model fit and the planet-moon model light curve model is refined. We find significant evidence in favor of the moon hypothesis. However, we notice that the resulting best fit to the light curve differs from the best fit in the paper of the original discoverers. We also find a different parameter distribution. This leads us to advising caution, since we would assume that a physical moon signature in the data would lead to roughly the same results independently of the exact detrending method used.

In Chapter 4, we find that the transit timing and duration shift is not as good an indicator for detecting exomoons as previously thought. We find however a possible other exomoon indicator: When planet and moon transit overlap, the observed transit depth increases. This increase follows a periodic pattern, determined by the length of the moon and planet period. We test this indicator on synthetic light curves and determine that this indicator can detect moon of Earth size for bright stars using Kepler-like telescopes.

The results of the previous chapters are discussed in Chapter 5. We also test machine learning using a simple test system, showing that machine learning might be able to detect Earth-sized exomoons. 



\section{Zusammenfassung}

Eines der nächsten großen Ziele für die Exoplanetenwissenschaft ist die Entdeckung eines eines Mondes um einen extrasolaren Planeten. In dieser Dissertation entwickele ich Methoden, um diese Monde zu entdecken und zu charakterisieren. Dabei liegt ein besonderer Fokus auf dem Mondkandidaten um den Exoplaneten Kepler-1625 b. Den Hauptteil dieser Dissertation bilden zwei Paper zu diesem Exomondkandidaten sowie ein kurzes Kapitel zu einem neuen Exomondindikator, den wir entwickelt haben. Diese Themen und die Ergebnisse sind im folgenden kurz zusammengefasst.

Kapitel 2 widmet sich dem postulierten Mondkandiaten um den extrasolaren Planeten Kepler-1625 b. Wir testen die Verlässlichkeit dieses Fundes, indem wir Transitsignale von Planeten mit und ohne Mond in Teile der Lichtkurve injizieren, die zuvor keine Transitsignale aufwiesen. Wir finden in ca. 40\% der Lichtkurven, in die ein zusätzliches Mondsignal injiziert wurde, einen Mond. Allerdings finden wir auch in ca. 10\% der Fälle, in denen kein Mondsignal injiziert wurde, einen Mond. Dies ist eine hohe Falsch-PositivRate, wenn wir uns vor Augen führen, dass bei hunderten von extrasolaren Planeten nach Monden gesucht wurde. Wir analysieren auch die ursprüngliche Lichtkurve von Kepler1625 und finden, dass die Mondhypothese leicht bevorzugt wird.

In Kapitel 3 kehren wir zum Mondkandidaten um Kepler-1625 b zurück, nachdem ein weiterer Transit vom Hubble-Teleskop beobachtet wurde. Wir suchen sowohl in der Lichtkurve, die von den Entdeckern des Mondkandidaten bereitgestellt wurde, als auch in der Lichtkurve, die wir selber bereinigt haben, nach der Existenz eines Mondes. Im Gegensatz zum ersten Paper wird nun gleichzeitig der Langzeittrend und die Transitparameter gefittet. Die Analysen beider Lichtkurven zeigen sehr starke statistische Anzeichen für die Existenz eines Mondes. Allerdings finden wir andere am besten passende Modellichtkurven und eine andere Parameterverteilung als in dem Paper der Entdecker. Aus diesem Grund raten wir zur Vorsicht, da wir erwarten würden, dass ähnliche Analysen der Beobachtung eines echen Mondes zu ähnlichen Ergebnissen kommen sollten.

In Kapitel 4 untersuchen wir den Effekt, den der Mondtransit auf den kombinierten Planeten- und Mondtransit hat. Es stellt sich heraus, dass die vorhergesagte Verschiebung des Zeitpunktes des Transits und die Veränderung der Transitdauer durch die Bewegung des Planten um den Planet-Mond-Schwerpunkt kein guter Indikator für die Existenz eines Exomondes ist, da der Effekt teilweise durch einen gegenläufigen Effekt des Mondtransits auf den gemessenen Transitzeitpunkt und -dauer ausgeglichen wird. Wir stellen einen neuen Exomondindikator vor: Je nach Planet-Mond-Geometrie ändert sich die Transittiefe über eine Reihe von Transits. Diese Veränderung könnte für erdgroße Monde beobachtet werden.

In Kapitel 5 werden die Ergebnisse der vorangehenden Kapitel diskutiert. Außerdem erfolgt ein Ausblick, mit einem Schwerpunkt auf der Benutzung von maschinellem Lernen, um Exomondkandidaten effizient zu finden. 



\section{Preamble}

Parts of this thesis has the form of a cumulative thesis ("Kumulative Dissertation") as specified in the GAUSS Promotionsordnung, § 10 (3). Chapters 2 and 3 are reproductions of the following papers:

Paper I: Revisiting the exomoon candidate signal around Kepler-1625 b: Rodenbeck, K., Heller, R, Hippke, M., Gizon, L. 2018, Astronomy E Astrophysics, 617, A49

Paper II: An alternative interpretation of the exomoon candidate signal in the combined Kepler and Hubble data of Kepler-1625: Heller, R., Rodenbeck, K., Bruno, G. 2019, Astronomy $\mathcal{E}$ Astrophysics, 624, A95 



\section{Introduction}

Up to this date, thousands of extrasolar planets (planets around stars other than our sun, commonly called exoplanets) have been discovered, with some of these planets being as small as our Earth. At the same time, we know of over 170 moons in our Solar System, with the largest moon, Ganymede, having a radius almost half that of Earth. It is not unreasonable to assume that at least some exoplanets have moons of their own (commonly called exomoons), even if we do not know anything about the possible size of these exomoons. One might conclude that the exomoon community is tantalizingly close to the first undisputed discovery of an exomoon. Indeed, recently an exomoon candidate has been announced by Teachey et al. (2018) around the exoplanet Kepler-1625 b. This candidate is not yet confirmed and its existence is an ongoing topic of debate (Teachey and Kipping 2018, Rodenbeck et al. 2018, Heller et al. 2019) as well as the main topic of this thesis. In this chapter I give a short introduction into exoplanets, exomoons, their properties, and the main methods used to detect them.

\subsection{Exoplanets}

\subsubsection{Overview}

Exoplanetary science, the study of exoplanets, is one of the youngest subfields of astrophysics, with the first discovery of an exoplanet less than 30 years ago. The first exoplanet orbiting a main-sequence star was discovered in 1995 around 51 Pegasi (Mayor and Queloz 1995). It has a mass of at least ${ }^{1}$ half that of Jupiter and orbits its star every 4 days. Since then almost 4000 exoplanets have been found and confirmed, with another 2500 candidates waiting to be confirmed or rejected as exoplanets.

Mayor et al. (2011) estimate that around 50\% of all stars have at least one planet, rising to about $70 \%$ for $\mathrm{G}$ and $\mathrm{F}$ stars (i.e. sun-like or a bit more massive). Petigura et al. (2013) determined that around a quarter of sun-like stars have a planet with a radius between 1 and 2 Earth radii $\left(R_{\oplus}\right)$ orbiting with a period between 5 and 100 days.

Most of the planets discovered so far have periods between 1 and 100 days, with a radius between 1 and 4 Earth radii (see Fig. 1.1). This means that most of the discovered exoplanets have a size smaller than Neptune, which, without accurate mass measurements, makes it difficult to determine if those planets are upscaled Earth analogues (superEarths) or downscaled Neptune analogues (mini-Neptune). Many of the giant planets confirmed as exoplanets so far are roughly Jupiter-sized planets, close to their host star, with orbital period of only a few days. These objects, named Hot Jupiters, form a distinct

\footnotetext{
${ }^{1}$ The radial velocity method used to detect it can only yield a lower bound on the mass, see Sec. 1.1.2.2.
} 


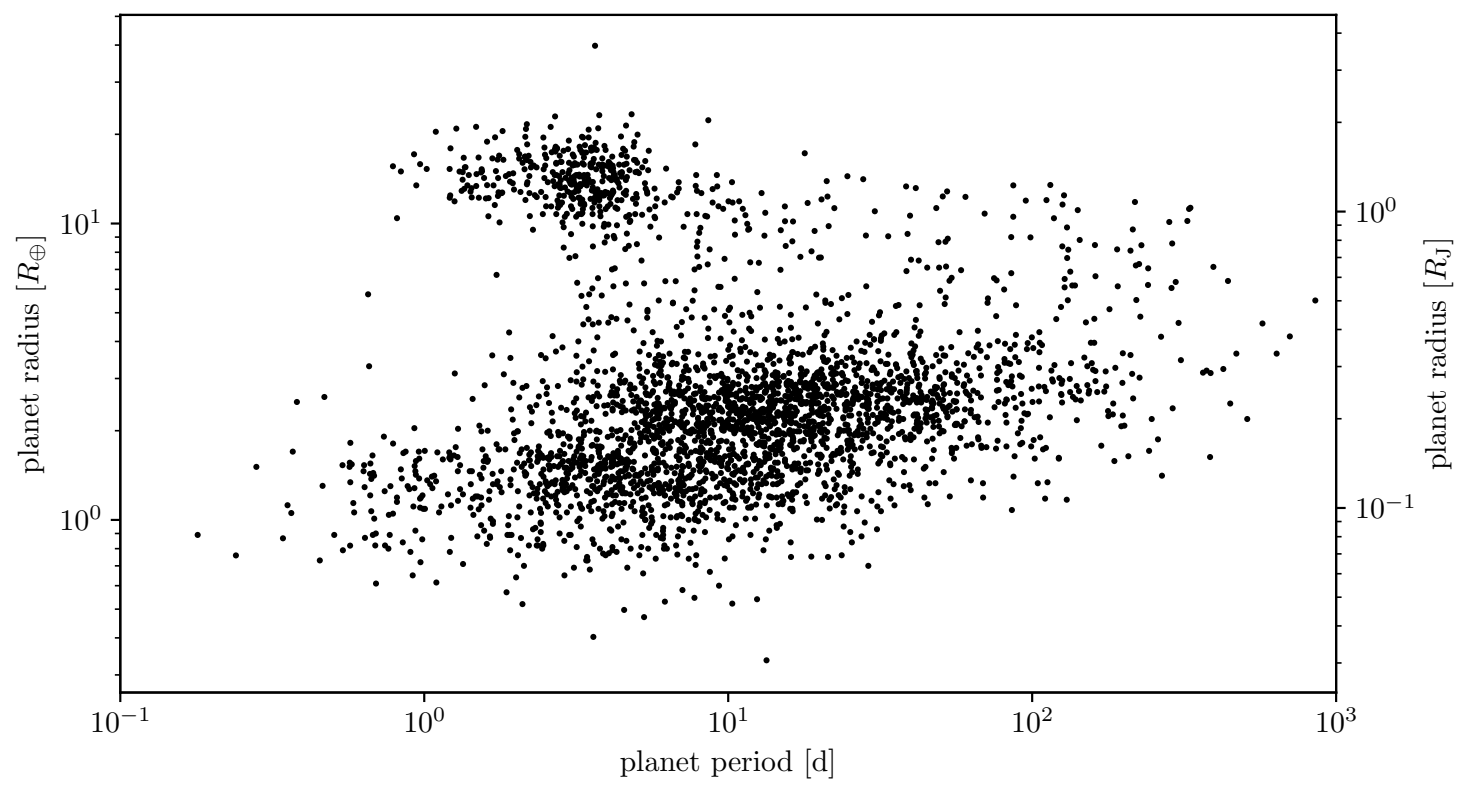

Figure 1.1: Radius over orbital period of the confirmed exoplanets for which both values are know, and whose period is smaller than $1000 \mathrm{~d}$. The Hot Jupiters (planets with large radii at very short periods) form a distinct sub-group separated from the other exoplanets. The other exoplanets mostly have radii between 1 and $4 R_{\oplus}$, making them so-called super-Earths or mini-Neptunes. Data taken from http://exoplanetarchive.ipac. caltech.edu.

sub-group in the period-radius diagram (and indeed in all other diagrams relating size and distance to the star) and have no equivalent in our Solar System, presenting a new class of planets that we did not know existed before the discovery of the first exoplanet.

\subsubsection{Detection Methods}

There are two main methods how exoplanets are detected and characterized: The so-called transit method and the so-called radial velocity (RV) method.

\subsubsection{The Transit Method}

The transit method works by detecting the dimming of a star when an exoplanet belonging to that star passes in front of the star. By fitting a transit model to the observed dimming, we can determine some of the planet's parameters. The profile of this dimming depends on the distance of the exoplanet to the star's center, projected along our line-of-sight over time, the ratio of the planet's and star's radius $r_{\mathrm{p}}$ and the limb-darkening profile (stars appear less bright towards the edge of the observed disk) of the star (Mandel and Agol 2002). The planet's projected distance $z_{\mathrm{b}}$ to the star's center is determined by the planet's orbit around its host star. The parameter $z_{\mathrm{b}}$ is often expressed in units of stellar radii $R_{\star}$ and for circular orbits is given by 


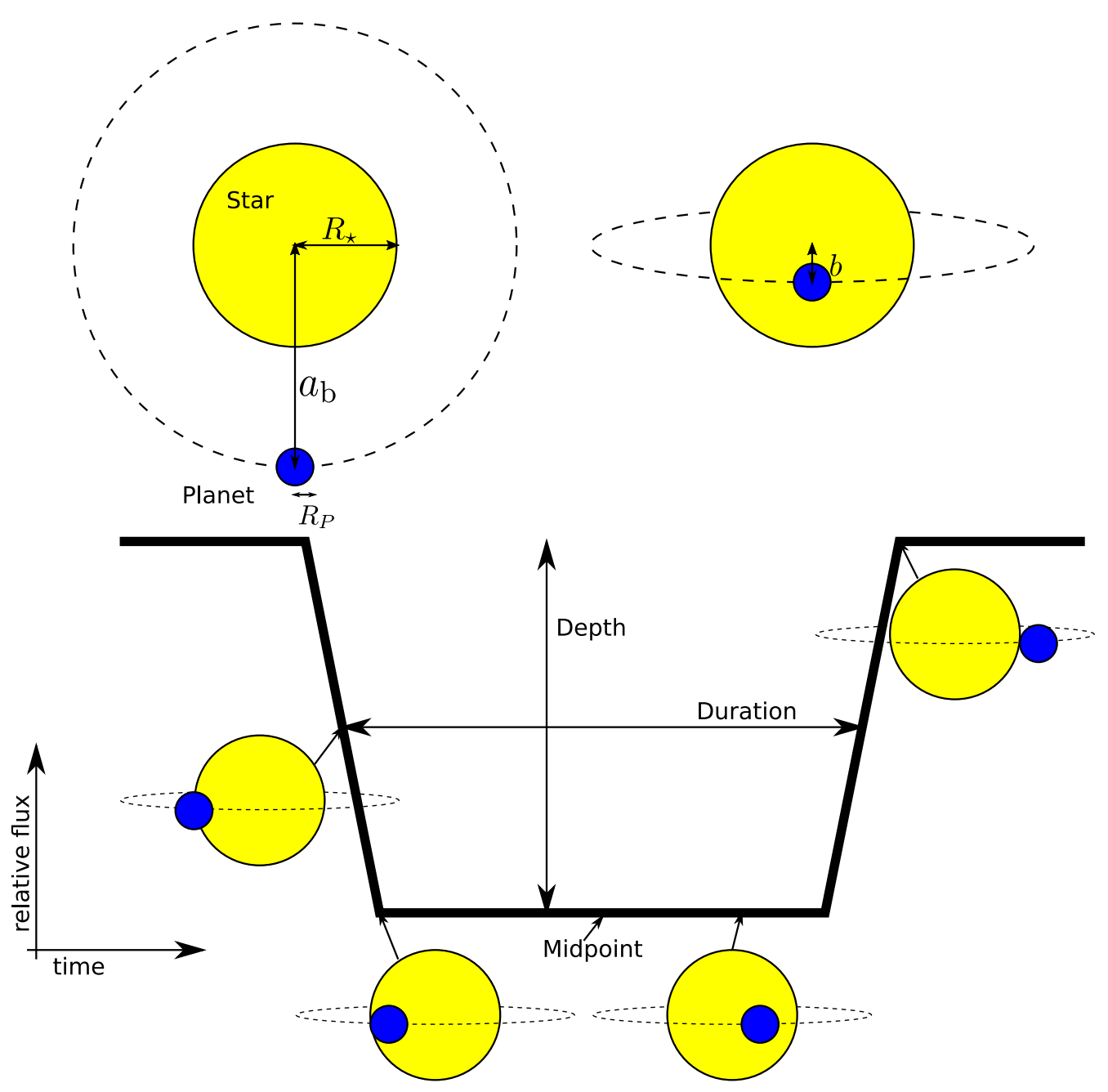

Figure 1.2: Schematic diagram showing a planetary transit. In the top part the orbital configuration of the star and planet is shown. On the left from the top (face-on) and on the right from the observers point of view (edge-on). The bottom part shows the resulting transit, as well as the orbital configurations corresponding to certain points during the transit. For clarity, limb-darkening is neglected in this figure.

$$
z_{\mathrm{b}}\left(t, \frac{a_{\mathrm{b}}}{R_{\star}}, b, P_{\mathrm{b}}, t_{0, \mathrm{~b}}\right)=\sqrt{\left(\frac{a_{b}}{R_{\star}} \sin \frac{2 \pi\left(t-t_{0, \mathrm{~b}}\right)}{P_{\mathrm{b}}}\right)^{2}+\left(b \cos \frac{2 \pi\left(t-t_{0, \mathrm{~b}}\right)}{P_{\mathrm{b}}}\right)^{2}},
$$

where $P_{\mathrm{b}}$ is the orbital period of the planet, $a_{\mathrm{b}}$ the semi-major axis of the planet, $t_{0, \mathrm{~b}}$ the midpoint of one of the transits and $b$ the so-called impact factor, the smallest distance of the planet to the star's center in units of the stellar radius $R_{\star}$. Planets only cause a transit if they are in front of the star, not behind (although the secondary transits caused by the blocking of star light reflected on the planet can yield information about the exoplanets atmosphere), which has to be taken into account when calculating the transit light curve from $z_{\mathrm{b}}$. Fig. 1.2 shows the relation between an exoplanet's orbit and the resulting transit light curve. 


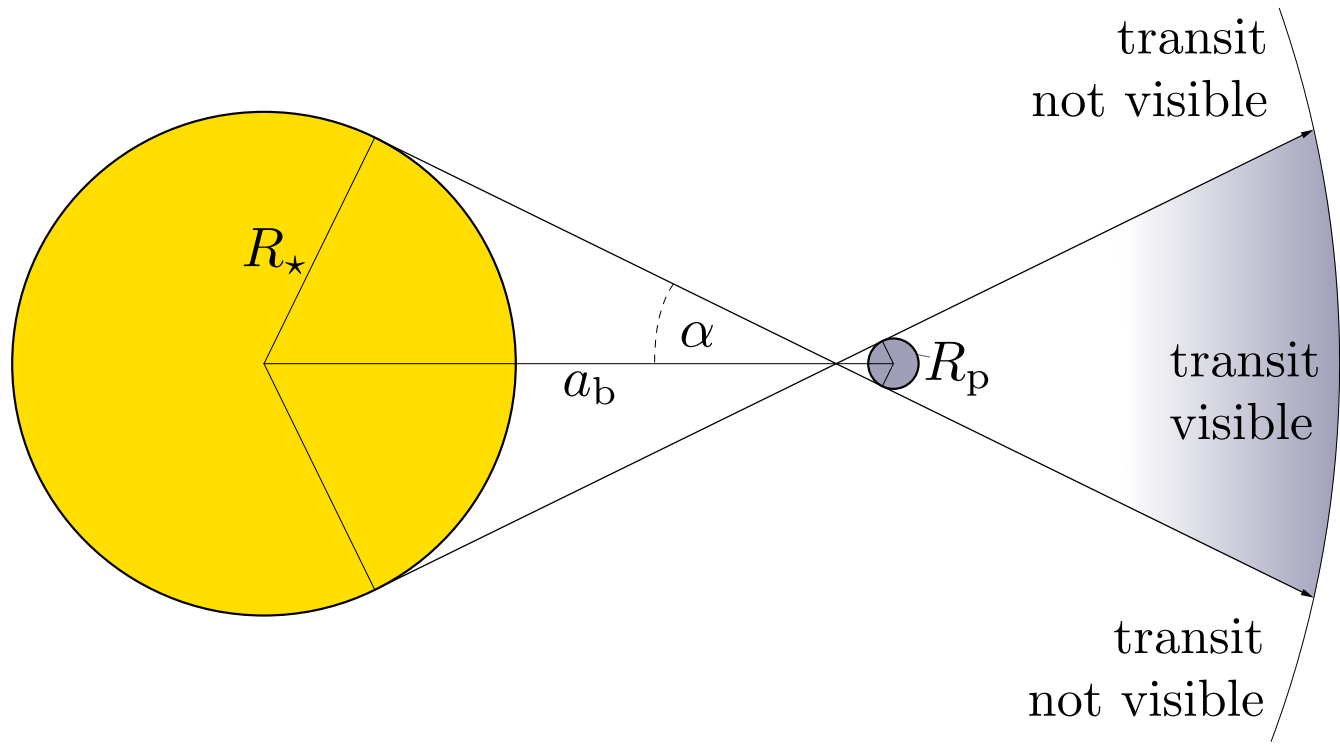

Figure 1.3: Diagram of the geometry determining from which angles observers can see a transit of the planet (grey body) in front of the star (yellow body).

If a dimming of a star is caused by a transit it will happen again and again always after one orbital period $P_{\mathrm{b}}$. The periodicity of this dimming is one of the major indicators that the dimming is in fact caused by an orbiting planet and not some other astrophysical or instrumental effect.

Using the transit method we can only detect transits for which the orbital configuration is such that the planet is in front of the star during at least one point of the orbit, i.e. $z_{\mathrm{b}}(t)<$ $1+r_{\mathrm{p}}$ at some time $t$, where $r_{\mathrm{p}}$ is the ratio between the star's radius $R_{\star}$ and the planet's radius $R_{\mathrm{p}}$. Fig. 1.3 shows from which angle $\alpha$ above and below the planet's orbital plane a transit can be observed. The angle $\alpha$ can be calculated as $\alpha=\sin ^{-1}\left[\left(1+r_{\mathrm{p}}\right) R_{\star} / a_{\mathrm{b}}\right]$. Assuming the direction from which a potential observer looks at the star is distributed uniformily, the probability of being able to observe a transit is given by

$$
P_{\mathrm{Tr}}=\frac{1}{2} \int_{-\alpha}^{\alpha} \cos \theta \mathrm{d} \theta=\frac{\left(1+r_{\mathrm{p}}\right) R_{\star}}{a_{\mathrm{b}}} .
$$

For a Jupiter-sized planet in a $3.7 \mathrm{~d}$ orbit around a Sun-sized star this would mean a $10 \%$ probability of observing a transit of this planet. For an Earth-sized planet in a 1 year orbit this probability drops to $0.5 \%$. For a derivation of this probability taking into account the eccentricity of the planet's orbit see Barnes (2007).

The discrepancy between the expected transit time and the observed transit time (transit timing variation, TTV) can be used to detect non-transiting planets (see e.g. Ballard et al. 2011) and determine the mass of the other planets in the same planetary system (see e.g. Gillon et al. 2017).

The Kepler space telescope (Borucki et al. 2010), using the transit method, has conducted the largest search for exoplanets to this date: It observed brightness variations of 200000 stars for 4 years from 2009 to 2013 (Twicken et al.2016). 


\section{AObserver}

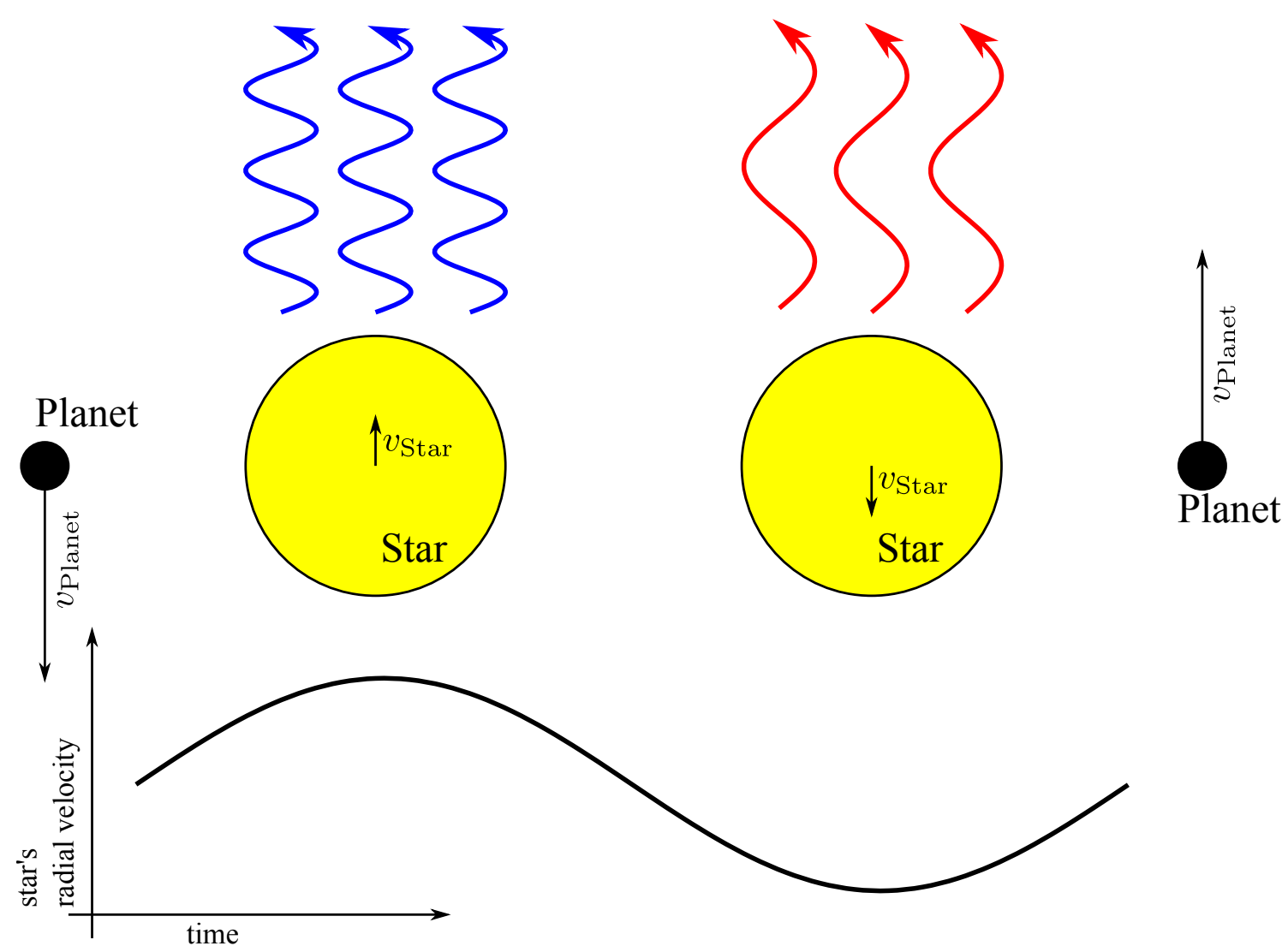

Figure 1.4: Schematic overview on how planets are detected using the radial velocity (RV) variations of the host star. The star orbits the star-planet barycenter, changing its velocity towards the observer. This causes a red- or blue-shift of the emitted light.

\subsubsection{The Radial Velocity Method}

The radial velocity (RV) method works by measuring a star's velocity along our lineof-sight. Just as the planet is gravitationally attracted to the star, the star is attracted to the planet with the same force. Due to the star's much higher mass, the resulting acceleration and velocity is much smaller than that acting on the planet. This velocity variation of the star can be detected by measuring the red- and blue-shift of the star's light (see Fig. 1.4). This red- and blue-shift, known as Doppler shift, is detected by measuring the shift of the absorption lines in the stellar spectrum. The RV method is sensitive only to movement of the target star towards and away from the observer. This means that the RV method becomes less and less sensitive to the movement of the star due to the planet as the inclination $i$, the angle between an observer's line-of-sight and the planet's orbital plane, grows. If the orbital plane of the planet is perpendicular to the line-of-sight, the RV method can not detect any movement of the star due to the planet's gravitational attraction at all. Since the inclination of the planet is not known and only one part of the 
stellar velocity can be measured by the RV method, it can only provide a minimum mass of a planet, often denoted $M_{\mathrm{p}} \sin i$.

The amplitude $K$ of a RV signal caused by a planet in a circular orbit is given by (e.g. Cumming et al. 1999)

$$
K=\left(\frac{2 \pi G}{P_{\mathrm{b}}}\right)^{1 / 3} \frac{M_{\mathrm{p}} \sin i}{\left(M_{\star}+M_{\mathrm{p}}\right)^{2 / 3}},
$$

where $P_{\mathrm{b}}$ is the orbital period of the planet, $G$ is the gravitational constant and $M_{\star}$ the star's mass. For Jupiter in its orbit around the Sun the expected amplitude, assuming $i=0$, is $12.5 \mathrm{~m} / \mathrm{s}$ and for the Earth it is $0.09 \mathrm{~m} / \mathrm{s}$.

While the first exoplanet has been found through RV measurements, the largest part of exoplanets detected so far were found by the transit method. This is due to the fact that the transit method uses photometry, which means that we can observe many stars at once using cameras, like the Kepler space telescope did.

\subsection{Exomoons}

Moons are natural satellites around planets and dwarf-planets. Exomoons are moons around extrasolar planets.

Despite there being 20 times as many moons as planets in our Solar System, there has not been one confirmed exomoon yet. Exomoons might play an important part in the search for habitable bodies around stars other than the Sun (Williams et al.|1997, Zollinger et al. 2017), since the habitable zone around a host star is expanded outwards if the moon is heated by tidal interactions with its planet (Reynolds et al. 1987, Dobos et al. 2017).

\subsubsection{Moons in our Solar System}

Our Solar System has 175 moons $s^{2}$ distributed around six of its eight planets. Most of these moons orbit around the two biggest gas giants Jupiter (79) and Saturn (53), with the two smaller gas planets having 27 (Uranus) and 13 (Neptune) moons. Earth has one moon, the Moon, and Mars has two small moons. Additionally, many of the dwarf planets have moons.

The first moons discovered aside from our own Moon were the four largest moons of Jupiter. They were observed in 1610 by Galileo Galilei, and are called, ordered by distance to Jupiter, Io, Europa, Ganymede and Callisto. These so-called Galilean moons have radii between 1560 and $2630 \mathrm{~km}$ and orbit Jupiter with periods between 1.7 and $17 \mathrm{~d}$. The largest moon of our Solar System is Ganymede, the third of the Galilean moons, with a radius of about $2630 \mathrm{~km}$, followed closely by Titan, the largest moon of Saturn, with around $2570 \mathrm{~km}$.

The moons in the Solar System have formed through different processes. Some were formed in the tidal debris disks of the gas planets (e.g. the Galilean moons, see Crida and Charnoz (2012)), some were captured (e.g. Triton, a moon of Neptune, see Agnor and Hamilton (2006)). Earth's Moon may have been formed by an impact of a Mars-sized body early in Earth's history (Cameron and Ward 1976).

${ }^{2}$ https://solarsystem.nasa.gov/moons/in-depth/ 


\subsubsection{Moons as Tracers of Formation History}

With the discovery of the Hot Jupiters and the planet distribution that Kepler revealed, as well as the discovery and characterization of multi-planet systems, it has become clear that extrasolar planetary systems can have completely different structures compared to our Solar System. The moon distribution outside the Solar System might also be completely different from the one in the Solar System, especially around planets for which we do not have equivalents in the Solar System (Hot Jupiters, Warm Neptunes). The existence or non-existence of moons around certain planet types can give valuable insight into the formation history of their host systems.

A detailed history of the formation and evolution of the moons in the Solar System can help constrain planetary formation and evolution models. Canup and Ward (2002), Heller et al. (2015) use the composition of the Galilean moons to constrain the temperature distribution in the disk formed around the early Jupiter due to accretion. Similar studies would be possible for extrasolar systems once exomoons are detected. Namouni (2010) conclude that moons around gas giants might not survive the migration inwards, which is one of the main scenarios for the formation of Hot Jupiters. A detection of moons around Hot Jupiters could help to exclude the migration model as a formation model.

\subsubsection{Habitability of Moons}

Exomoons present a possible place where life might develop, given the right conditions (for a review on habitability of exomoons see Heller et al.(2014)). Extrapolating from the bodies in the Solar System where life might develop, there might even be more habitable exomoons than exoplanets.

The most important condition for life is the presence of liquid water. This requires an energy source to keep the water liquid over long periods of time. For planets the most important source of energy is their star's radiation. The region around a star where the star's radiation keeps water liquid is called the Habitable Zone (HZ). In the Solar System, Earth is the only planet with large quantities of liquid water, with $70 \%$ of its surface covered in water. Moons can be tidally heated by interaction with their parent planet, which can extend the region around the star where liquid water is possible (Reynolds et al. 1987, Dobos et al. 2017). Four moons in the Solar System might harbor large bodies of liquid water: Europa, Enceladus, Ganymede and Callisto. Europa, under a $10 \mathrm{~km}$ layer of ice, has a layer of liquid water between 10 and $100 \mathrm{~km}$ deep. The energy to maintain this ocean comes from the tidal interaction with Jupiter. Enceladus might contain localized pockets of a subsurface liquid water. Ganymede and Callisto might also posses oceans with a depth of around $300 \mathrm{~km}$ (Spohn and Schubert 2003). The evidence for subsurface water on Europa and Enceladus are plumes of water ice they eject. Measurements of Ganymede's and Callisto's density and moment of inertia are in agreement with subsurface oceans.

Another important requirement for life is a source of energy usable by organisms. The most commonly used source for energy for organisms on Earth is photosynthesis. This would require moons in the habitable zone around its star to have an atmosphere to enable exposure to sun light. Williams et al. (1997) find that for a moon in the HZ this requires a moon mass larger than $0.1 M_{\oplus}$ (which is roughly the mass of Mars) and the presence of 
a magnetic field to protect the moon from particles in the planet's magnetosphere. Titan is unique among the moons in the Solar System in that it has a dense atmosphere, which has even a stronger pressure than Earth at ground level. Its atmosphere is dominated by nitrogen with $98 \%$, with most of the rest made up of methane and hydrogen (Coustenis et al.2007); it is also so dense that very little light reaches the ground. Titan does not lay in the HZ. At a temperature of around $100 \mathrm{~K}$, life on Titan can not be based on water, but instead would have to be on the basis of hydrocarbons. Life on moons with subsurface oceans like Europa could extract energy from volcanic vents, similar to some organisms on Earth's ocean floor.

The occurrence rate of moons massive enough to hold an atmosphere enabling photosynthesis is unknown (and in fact zero in the Solar System). It might be possible that more massive moons form around super-Jovian exoplanets (planets more massive than Jupiter) and brown dwarfs (although it would be a matter of definition if those bodies would be counted as moons or planets). Even though there are models how such massive moons might be formed (Heller et al. 2014, Williams 2013), in the end only detections of such bodies can give us an estimate on the occurrence rate of massive moons.

Moons might also play a role in the habitability of their planets, since they can help stabilizing the obliquity of the planet, as is the case for Earth (Laskar et al. 1993).

\subsubsection{Detection and Characterization Methods}

There are various ways how an exomoon could be detected (for a review see Heller 2018b).

Using transit photometry, there are two effects caused by the existence of a moon: Firstly, the effect of the moon on the planetary transit, and secondly, the transit of the moon itself in front of the star.

The planet and moon orbit their combined center of mass, the barycenter, both with the same period $P_{\mathrm{s}}$, but with very different semi-major axes. The ratio between these two semi-major axes is determined by the mass ratio of planet and moon.

This movement of the planet around the barycenter affects the transit shape and position (see Fig. 1.5): The planet can be ahead or behind in its orbit around the star, compared to the barycenter, causing a shift in the transit midpoint (Sartoretti and Schneider 1999), called the transit timing variation (TTV), and the planet can be faster or slower compared to the barycenter's movement around the star, causing a longer or shorter transit duration (Kipping 2009), called the transit duration variation (TDV). Due to the geometry of the planet's orbit around the barycenter, the TTV and TDV curves caused by a moon are sinusoidal for a moon with a circular orbit, and are offset relative to each other by a phase difference of $90^{\circ}$.

Heller et al. (2016b) propose to search for exomoons signatures in the TTV-TDV parameter space: The TTVs and TDVs for a series of transits, plotted against each other, form an ellipse in the TTV-TDV space if they are caused by the presence of an exomoon. This is due to the $90^{\circ}$ phase shift mentioned above. In Chapter 4 we re-evaluate this detection method.

The moon can also have its own transit in front of the star (but does not have to, depending on the orbital configuration, see Martin et al. (2019)). Just like for a planetary transit, the depth of the transit is determined by the size of the moon. Due to the moon's 
a) Maximal TTV

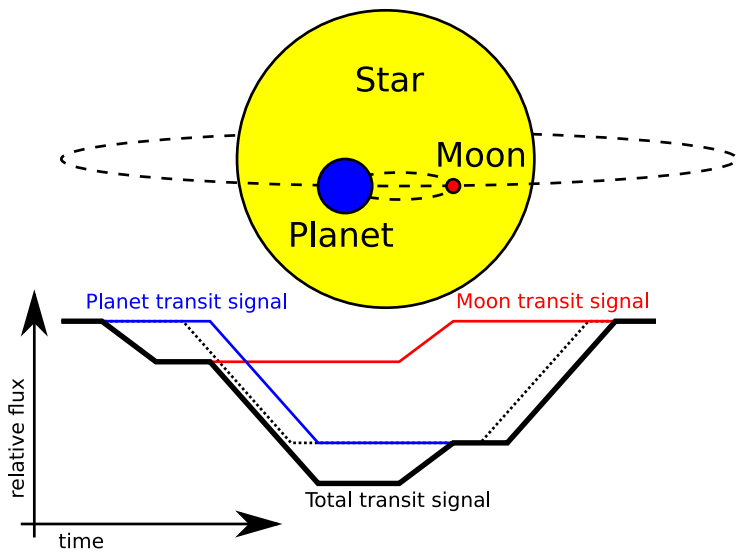

b) Maximal TDV

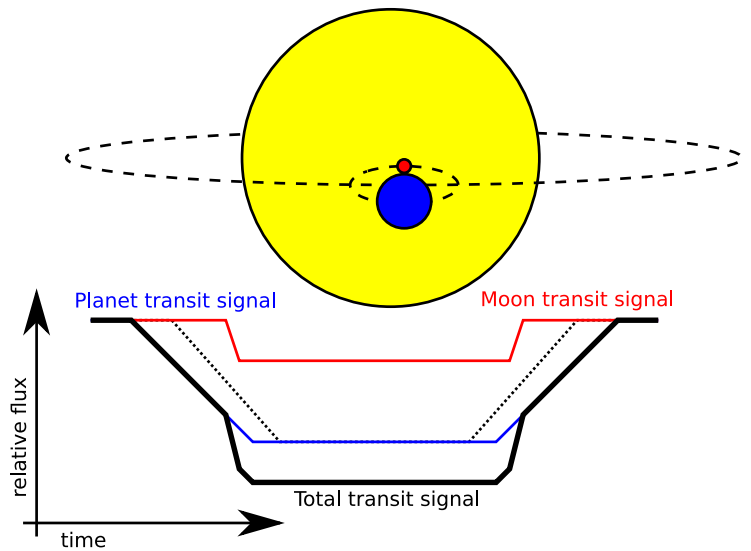

Figure 1.5: Schematic overview how the movement of planet and moon affect the shape and timing of the planet and moon transit. The left half shows a configuration that causes maximal TTV: The planet is left of the planet-moon barycenter, meaning its transit (blue) is shifted backwards compared to a undisturbed transit (dashed line). The moon's transit (red) happens earlier compared to the barycenter. The right half shows a configuration causing maximal TDV: The planet and moon transit happen simultaneously, but due to the planet's slower velocity, its transit is prolonged, compared to the undisturbed one. The moon's transit is shorter than the undisturbed one, due to its higher velocity.

movement around the planet-moon barycenter, the transit shape is distorted compared to the shape one would expect from the barycenter's orbital speed. The same rules as for the planet's shift apply, but more exaggerated: Depending on the orbital phase of the moon around the barycenter, a TTV or TDV is caused, but at a much higher amplitude than for the planet. For moons with shorter periods comparable to the transit duration, the transit shape of the moon can drastically alter. It is for example possible that the moon causes two transits per barycenter orbit, if the moon's total velocity becomes negative compared to the orbital movement of the planet-moon system.

Heller (2014), Heller et al. (2016b) suggest to search for exomoon signatures in the phase-folded transit light curves (where the flux level is not plotted against time, but against the orbital phase of the planet). In the presence of a moon, the average light curve on each side of the planet transit shows a small drop in brightness which is related to the moon's size and semi-major axis (which the authors call Orbital Sampling Effect, OSE). In a similar approach, Simon et al.(2012) suggest that the scatter of the phase folded light curve on the sides of the transit increases in the presence of a moon.

Both of these effects can be combined into a complete dynamical photometric model, comprising of both the planetary and the moon transit (Kipping 2011, Rodenbeck et al. 2018). The same general idea as for a single planet transit applies: The projected distances of the planet $\left(z_{\mathrm{p}}\right)$ and moon $\left(z_{\mathrm{s}}\right)$ to the stars center at a given time are calculated, and together with the radius ratios $r_{\mathrm{p}}$ and $r_{\mathrm{s}}$ and a limb-darkening profile the relative dimming is calculated. The projected distance to the star's center is composed of the projected distance of the planet-moon barycenter $\left(z_{\mathrm{b}}\right.$, the same as for an undisturbed planet) and the projected distance due to the orbital configuration of planet and moon. Finally, the possi- 
bility of an occultation (the planet being in front of the moon as seen from the observer, or vice-versa) needs to be taken into account.

The radial velocity method can not be used directly to detect exomoons: If the distance between the planet and moon is much smaller than the distance between the planet-moon system and the star, the combined gravitational force of a planet and moon on their star is very close to the gravitational force of a planet with the combined mass of the previous planet and moon. However, the passing of the moon in front of the star can cause a variation in the measured radial velocity (the so-called Rossiter-McLaughlin effect), just like the passing of the planet can (Zhuang et al.2012). If a planet can be directly imaged, it might be possible to detect the motion of the planet around the planet-moon barycenter with Doppler measurements (Vanderburg et al. 2018a).

While there are many difficulties trying to detect an exomoon, there is one big advantage compared to the search for exoplanets: We know that exomoons orbit their planets. This massively reduces the amount of light curves that have to be searched (only those with a detected planet), and also reduces the regions in a light curve where a moon signal may be found. The maximum distance a moon can be separated from its planet is proportional to the distance from the planet at which the force of the star on the moon and the centripetal force due to the planets orbit around the star equal the force of the planet on the moon. This distance is called the Hill radius $R_{\mathrm{Hill}}$.

The Hill radius depends on the distance between a star and its planet $a_{\mathrm{b}}$ and their mass ratio $M_{p} / M_{\star}$ :

$$
R_{\text {Hill }}=a_{\mathrm{b}}\left(\frac{M_{\mathrm{p}}}{3 M_{\star}}\right)^{1 / 3} .
$$

A moon's orbit is stable up to a certain fraction $\eta$ of the Hill radius from 0.5 for prograde moons and up to 1 for retrograde moons (Domingos et al. 2006). This means that the maximum time $t_{\text {Hill }}$ that a moon transit can be separated from the planet transit is given by

$$
t_{\text {Hill }}=\eta \frac{R_{\text {Hill }}}{v_{\text {orbit }}}=\eta \frac{P_{\mathrm{b}}}{2 \pi}\left(\frac{M_{\mathrm{p}}}{3 M_{\star}}\right)^{1 / 3}
$$

For a hypothetical Earth transit in front of the sun as seen from an outside observer and with $\eta=0.5$, this results in a $0.3 \mathrm{~d}$ window each side of the transit where a possible moon signal might occur. This time window grows to $23.5 \mathrm{~d}$ for a Jupiter transit.

\subsubsection{Surveys and Searches so far}

The first search for planetary satellites was performed by Brown et al. (2001) using Hubble photometry of four planetary transits of HD 209458. They exclude the existence of moons larger than $1.2 R_{\oplus}$ around that planet at a $3 \sigma$ level.

The Hunt for Exomoons with Kepler (HEK, Kipping et al. (2012)) survey searches for the most promising candidates for the detection of exomoons among the exoplanet candidates observed with the Kepler space telescope. Due to the limitations of the Kepler data (cadence, photometric precision), HEK focuses on very large moons (compared to the moons in the Solar System) of more than $0.1 M_{\oplus}$. The HEK survey also tries to 

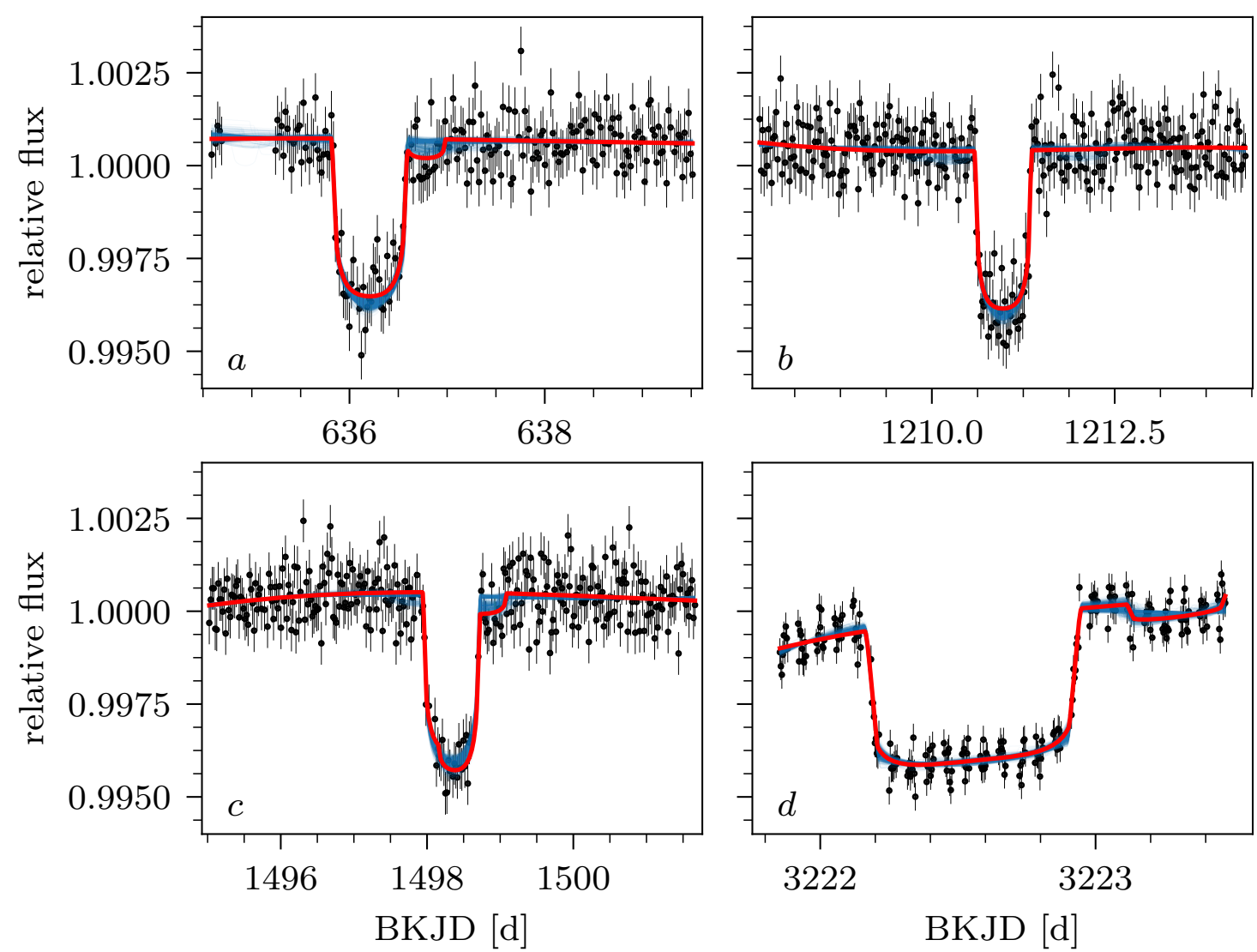

Figure 1.6: The four transits that have been observed of Kepler-1625 b, the host planet of the most recent exomoon candidate. The first three transits $(a, b$, and $c)$ were observed by the Kepler space telescope. The last transit $(d)$ was observed by the Hubble space telescope. For details see Chapter 3 and Fig. 3.2. The used light curves are taken from the published data of Teachey and Kipping (2018)

estimated the frequency of moons around exoplanets: In Teachey et al. (2018) the authors constrain the occurrence rate of a Galilean moon system around planets between 0.1 and 1 au from the star to under $39 \%$ (at a $95 \%$ confidence).

\subsubsection{The Exomoon Candidate around Kepler-1625 b}

In Teachey et al. (2018) the authors claimed to have found evidence of a Neptune-sized exomoon around the Jupiter-sized exoplanet Kepler-1625 b. The Kepler space telescope captured three transits in the light curve, with the resulting orbital period of $287 \mathrm{~d}$, which equates to a quite large Hill-stable region around the planet. The authors later presented a detailed analysis of the system (Teachey and Kipping 2018), with an additional transit, observed by the Hubble space telescope (see Fig. 1.6). The moon has a period of $22_{-9}^{+17} \mathrm{~d}$ and a semi-major axis of $0.98_{-0.13}^{+0.14}$ au. They find a mass ratio of moon and planet of $0.0141_{-0.0039}^{+0.0048}$, constraining it only weakly. One of the key findings is that the Hubble transit was 78 min to early, if the orbital period of the planet is only fitted using the Kepler transits. This may have been caused by the planet being disturbed by the moon 
(TTV) during one of the transits, although also other causes for this shift are possible, like the existence of another planet in the system.

\subsection{Data Sources for Exoplanet and Exomoon Studies}

The detection of exoplanets requires long-term observations of many stars, especially when using photometric data. This is due to the low probability of a single star to show signs of a planetary companion (see Sec. 1.1.2.1). Most of the exoplanet science conducted nowadays is based on data taken by the Kepler Space Telescope. Kepler was launched in 2009 and searched for transiting planets in front of 200000 stars during its main observation campaign from 2009 to 2013 (Borucki et al.|2010, Twicken et al.|2016). It has discovered about 4700 exoplanet candidates.

Both NASA and ESA have lined up several future space missions to study and detect exoplanets. Already launched, with the first scientific results published as of the writing of this theses, is NASA's Transiting Exoplanet Survey Satellite (TESS, Ricker et al. (2015)). TESS is an so-called all-sky survey, planned to cover $85 \%$ of the sky in its survey. The drawback of this plan is that TESS will observe parts of the sky for only a few weeks each. ESA's CHEOPS space mission (CHaracterising ExOPlanet Satellite, Broeg et al. (2013)) is scheduled to launch in fall 2019. It will observe transits of known exoplanet around bright stars with a high sampling rate of 1 minute. Simon et al.(2015) find that CHEOPS might be able to detect Earth-sized moons around Neptune sized planets with a $85 \%$ success rate. PLATO (PLAnetary Transits and Oscillations of stars, Rauer et al. (2014)) is another ESA space mission to search for exoplanets and will be launched around 2026. One of PLATO's main goals is to accurately characterize a huge number of planetary systems, including the interior composition of the planets. PLATO might also be able to detect large moons around the brightest observed stars 3

Once a exoplanet is detected, follow-up observations can be conducted by more powerful telescopes like the Hubble Space Telescope.

\subsection{Content of this Thesis}

The main part of this thesis is formed by two papers, which have been reproduced in Chapters 2 and 3, as well as a short chapter on a new exomoon indicator (Chapter 4).

In Rodenbeck et al. (2018) (Chapter 2), we perform an independent analysis of the available Kepler light curve of Kepler-1625 b, around which a exomoon candidate was discovered (Teachey et al. 2018). We also test the significance of this result by injecting model transits of a Kepler-1626 b-like planet system with and without moon into the out-of-transit light curve of Kepler-1625 b and rerun our analysis.

In Heller et al. (2019) (Chapter 3), we perform further analysis of Kepler-1625 b after another transit has been observed by the Hubble Space Telescope, using both the Hubble light curve published by Teachey and Kipping (2018) and our own version of it, which we independently extract and detrend.

\footnotetext{
${ }^{3}$ see the PLATO Definitions Study Report, http://sci.esa.int/jump.cfm?oid=59252
} 
In Chapter 4, we investigate the variations in the transit parameters caused by the existence of a moon. We propose the apparent planetary radius variation as a new exomoon indicator.

In Chapter 5 I discuss a few additional points relating to the two papers that form the main part of the thesis. I also present first results of using machine learning to detect exomoons. 



\title{
2 Paper I: Revisiting the exomoon candidate signal around Kepler-1625 $\mathbf{b}^{\text {ॠ }}$
}

\author{
Kai Rodenbeck ${ }^{1,2}$, René Heller ${ }^{2}$, Michael Hippke ${ }^{3}$, and Laurent Gizon ${ }^{2,1}$ \\ ${ }^{1}$ Institute for Astrophysics, Georg August University Göttingen, Friedrich-Hund-Platz 1, 37077 \\ Göttingen, Germany \\ ${ }^{2}$ Max Planck Institute for Solar System Research, Justus-von-Liebig-Weg 3, 37077 Göttingen, \\ Germany \\ ${ }^{3}$ Sonneberg Observatory, Sternwartestr. 32, 96515 Sonneberg, Germany
}

\begin{abstract}
Context: Transit photometry of the Jupiter-sized exoplanet candidate Kepler-1625 b has recently been interpreted as showing hints of a moon. This exomoon, the first of its kind, would be as large as Neptune and unlike any moon we know from the solar system.

Aims: We aim to clarify whether the exomoon-like signal is indeed caused by a large object in orbit around Kepler-1625 b, or whether it is caused by stellar or instrumental noise or by the data detrending procedure.

Methods: To prepare the transit data for model fitting, we explore several detrending procedures using second-, third-, and fourth-order polynomials and an implementation of the Cosine Filtering with Autocorrelation Minimization (CoFiAM). We then supply a light curve simulator with the co-planar orbital dynamics of the system and fit the resulting planet-moon transit light curves to the Kepler data. We employ the Bayesian information criterion (BIC) to assess whether a single planet or a planet-moon system is a more likely interpretation of the light curve variations. We carry out a blind hare-and-hounds exercise using many noise realizations by injecting simulated transits into different out-of-transit parts of the original Kepler-1625 light curve: (1) 100 sequences with three synthetic transits of a Kepler-1625 b-like Jupiter-size planet and (2) 100 sequences with three synthetic transits of a Kepler-1625 b-like planet with a Neptune-sized moon.

Results: The statistical significance and characteristics of the exomoon-like signal strongly depend on the detrending method (polynomials versus cosines), the data chosen for de-

*This chapter reproduces the article Revisiting the exomoon candidate signal around Kepler-1625 by K. Rodenbeck, R. Heller, L. Gizon and M. Hippke, published in Astronomy and Astrophysics 617, A49 (2018), reproduced with permission (C) ESO. Contributions: KR contributed to the analysis of the simulated light curves, to the interpretation of the results, and to the writing of the article.
\end{abstract}


trending, and the treatment of gaps in the light curve. Our injection-retrieval experiment shows evidence of moons in about $10 \%$ of those light curves that do not contain an injected moon. Strikingly, many of these false-positive moons resemble the exomoon candidate, that is, a Neptune-sized moon at about 20 Jupiter radii from the planet. We recover between about one third and one half of the injected moons, depending on the detrending method, with radii and orbital distances broadly corresponding to the injected values.

Conclusion: A $\triangle \mathrm{BIC}$ of -4.9 for the CoFiAM-based detrending is indicative of an exomoon in the three transits of Kepler-1625 b. This solution, however, is only one out of many and we find very different solutions depending on the details of the detrending method. We find it concerning that the detrending is so clearly key to the exomoon interpretation of the available data of Kepler-1625 b. Further high-accuracy transit observations may overcome the effects of red noise but the required amount of additional data might be large.

\subsection{Introduction}

Where are they? - With about 180 moons discovered around the eight solar system planets and over 3,500 planets confirmed beyond the solar system, an exomoon detection could be imminent. While many methods have indeed been proposed to search for moons around extrasolar planets (Sartoretti and Schneider 1999, Han and Han 2002, Cabrera, J. and Schneider, J. 2007, Moskovitz et al. 2009, Kipping 2009, Simon et al. 2010, Peters and Turner 2013, Heller 2014, Ben-Jaffel and Ballester 2014, Agol et al. 2015, Forgan 2017, Vanderburg et al. 2018b) ${ }^{1}$, only a few dedicated surveys have actually been carried out (Szabó et al. 2013, Kipping et al. 2013b a, 2014, Hippke 2015, Kipping et al. 2015, Lecavelier des Etangs et al. 2017, Teachey et al. 2018), one of which is the "Hunt for Exomoons with Kepler" (HEK for short; Kipping et al. 2012).

In the latest report of the HEK team, Teachey et al. (2018) find evidence for an exomoon candidate around the roughly Jupiter-sized exoplanet candidate Kepler-1625 b, which they provisionally refer to as Kepler- $1625 \mathrm{~b}$-i. Kepler-1625 is a slightly evolved G-type star with a mass of $M_{\star}=1.079_{-0.138}^{+0.100} M_{\odot}\left(M_{\odot}\right.$ being the solar mass), a radius of $R_{\star}=1.793_{-0.488}^{+0.263} R_{\odot}$ (with $R_{\odot}$ as the solar radius), and an effective temperature of $T_{\text {eff, } \star}=5548_{-72}^{+83} \mathrm{~K}$ (Mathur et al. 2017). Its Kepler magnitude of 15.756 makes it a relatively dim Kepler target. ${ }^{2}$ The challenge of this tentative detection is in the noise properties of the data, which are affected by the systematic noise of the Kepler space telescope and by the astrophysical variability of the star. Although the exomoon signal did show up both around the ingress/egress regions of the phase-folded transits (known as the orbital sampling effect; Heller 2014, Heller et al. 2016a) generated by Teachey et al. (2018) and in the sequence of the three individual transits, it could easily have been produced by systematic errors or stellar variability, as pointed out in the discovery paper.

The noise properties also dictate a minimum size for an exomoon detected around a given star and with a given instrument. In the case of Kepler-1625, we calculate the root-mean-square of the noise level to be roughly $700 \mathrm{ppm}$. As a consequence, any moon would have to be at least about $\sqrt{700 \mathrm{ppm}} \times 1.793 R_{\odot} \approx 5.2 R_{\oplus}\left(R_{\oplus}\right.$ being the Earth's

${ }^{1}$ For reviews see Heller et al. (2014) and Heller (2017).

${ }^{2}$ NASA Exoplanet Archive: https://exoplanetarchive.ipac.caltech.edu 

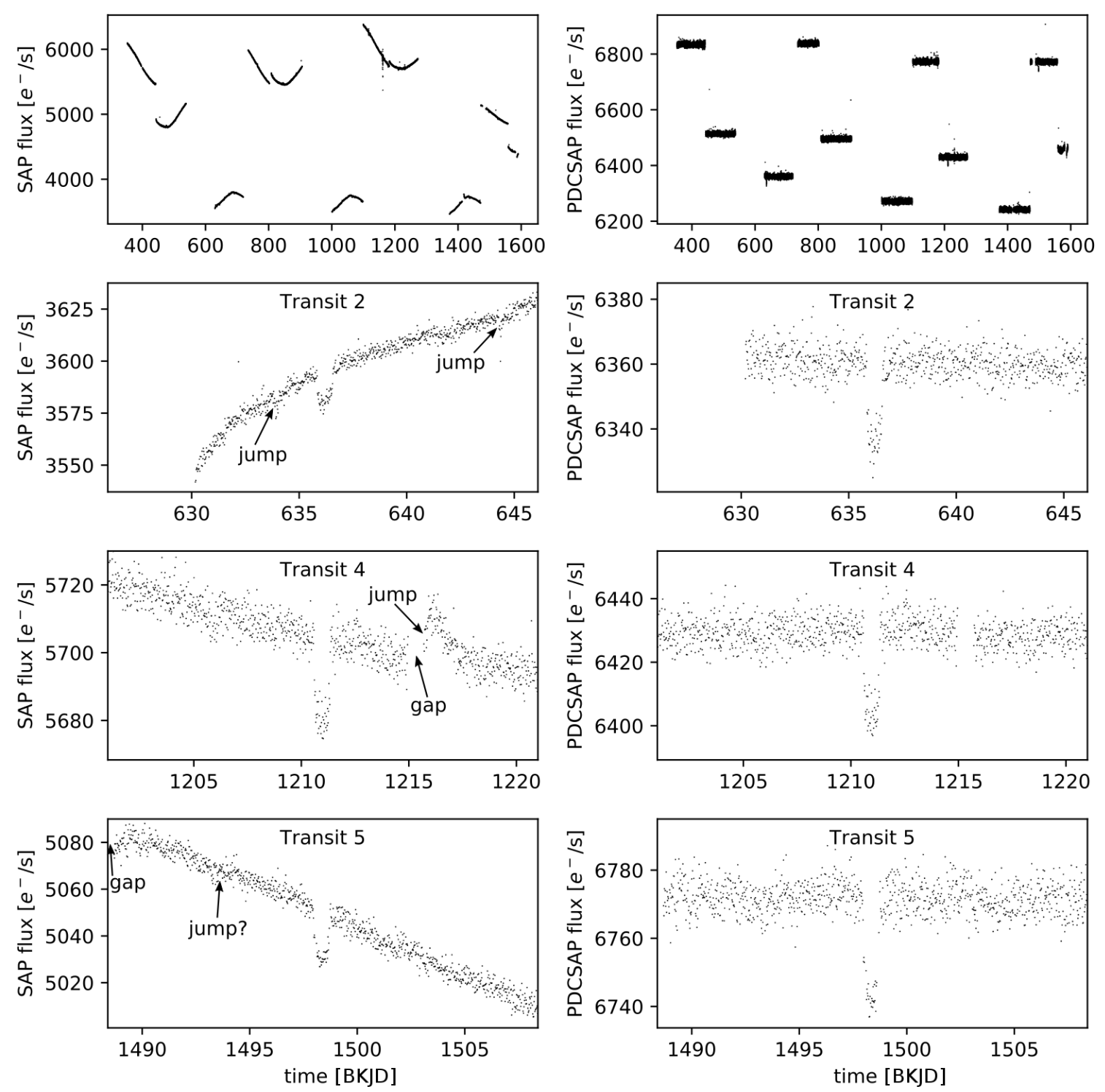

Figure 2.1: Kepler light curve of Kepler-1625. Left: Simple Aperture Photometry (SAP) flux. Right: Pre-search Data Conditioning Simple Aperture Photometry (PDCSAP) flux. The top panels show the entire light curves, respectively. The second, third, and fourth rows illustrate zooms into transits 2,4 , and 5 of Kepler- 1625 b, respectively. These transits were shifted to the panel center and $\pm 10 \mathrm{~d}$ of data are shown around the transit midpoints. Some examples of jumps and gaps in the light curve are shown. Time is given as a Barycentric Kepler Julian Date. 
radius) in size, about $30 \%$ larger than Neptune, in order to significantly overcome the noise floor in a single transit. The three observed transits lower this threshold by a factor of $\sqrt{3}$, suggesting a minimum moon radius of $\approx 3 R_{\oplus}$. In fact, the proposed moon candidate is as large as Neptune, making this system distinct from any planet-moon system known in the solar system (Heller 2018a).

Here we present a detailed study of the three publicly available transits of Kepler$1625 \mathrm{~b}$. Our aim is to test whether the planet-with-moon hypothesis is favored over the planet-only hypothesis.

First we develop a model to simulate photometric transits of a planet with a moon (see Sect. 2.2.2.2). Then we implement a detrending method following Teachey et al. (2018) and explore alternative detrending functions. Subsequently, we detrend the original Kepler-1625 light curve, determine the most likely moon parameters, and assess if the planet-with-moon hypothesis is favored over the planet-only hypothesis. Finally, we perform a blind injection-retrieval test. To preserve the noise properties of the Kepler-1625 light curve, we inject planet-with-moon and planet-only transits into out-of-transit parts of the Kepler-1625 light curve.

\subsection{Methods}

The main challenge in fitting a parameterized, noiseless model to observed data is the removal of noise on timescales similar or larger than the timescales of the effect to be searched for; at the same time, the structure of the effect must be untouched, an approach sometimes referred to as "pre-whitening" of the data (Aigrain and Irwin 2004). The aim of this approach is to remove unwanted variations in the data caused by, for example, stellar activity, systematic errors, or instrumental effects. This approach bears the risk of both removing actual signal from the data and of introducing new systematic variability. The discovery and refutal of the exoplanet interpretation of variability in the stellar radial velocities of $\alpha$ Centauri B serves as a warning example (Dumusque et al. 2012, Rajpaul et al.2016). Recently developed Gaussian process frameworks, in which the systematics are modeled simultaneously with stellar variability, could be an alternative method (Gibson et al. 2012). This has become particularly important for the extended Kepler mission (K2) that is now working with degraded pointing accuracy (Aigrain et al. 2015).

That being said, Teachey et al. (2018) applied a pre-whitening technique to both the Simple Aperture Photometry (SAP) flux and the Pre-search Data Conditioning (PDCSAP) flux of Kepler-1625 to determine whether a planet-only or a planet-moon model is more likely to have caused the observed Kepler data. In the following, we develop a detrending and model fitting procedure that is based on the method applied by Teachey et al. (2018), and then we test alternative detrending methods.

During Kepler's primary mission, the star Kepler-1625 has been monitored for 3.5 years in total, and five transits could have been observed. This sequence of transits can be labeled as transits 1, 2, 3, 4, and 5. Due to gaps in the data, however, only three transits have been covered, which correspond to transits 2, 4, and 5 in this sequence. Figure 2.1 shows the actual data discussed here. The entire SAP (left) and PDCSAP (right) light curves are shown in the top panels, and close-up inspections of the observed transit 2,4 , and 5 are shown in the remaining panels. The time system used throughout 
the article is the Barycentric Kepler Julian Date (BKJD), unless marked as relative to a transit midpoint.

A key pitfall of any pre-whitening or detrending method is the unwanted removal of signal or injection of systematic noise, the latter potentially mimicking signal. In our case of an exomoon search, we know that the putative signal would be restricted to a time-window around the planetary mid-transit, which is compatible with the orbital Hill stability of the moon. This criterion defines a possible window length that we should exclude from our detrending procedures. For a planet of ten Jupiter masses in a $287 \mathrm{~d}$ orbit around a $1.1 M_{\odot}$ star (as per Teachey et al. 2018), this window is about 3.25 days either side of the transit midpoint (see Appendix 2.A.

Although this window length is astrophysically plausible to protect possible exomoon signals, many other choices are similarly plausible, but they result in significantly different detrendings. Figure 2.2 illustrates the effect on the detrended light curve if two different windows around the midpoint of the planetary transit (here transit 5) are excluded from the fitting. We chose a fourth-order polynomial detrending function and a $7.5 \mathrm{~d}$ (blue symbols) or a $4 \mathrm{~d}$ (orange symbols) region around the midpoint to be excluded from the detrending, mainly for illustrative purposes. In particular, with the latter choice, we produce a moon-like signal around the planetary transit similar to the moon signal that appears in transit 5 in Teachey et al. (2018). For the former choice, however, this signal does not appear in the detrended light curve.

Teachey et al. (2018) use the Cosine Filtering with Autocorrelation Minimization (CoFiAM) detrending algorithm to detrend both the SAP and PDCSAP flux around the three transits of Kepler- $1625 \mathrm{~b}$. CoFiAM fits a series of cosines to the light curve, excluding a specific region around the transit. CoFiAM preserves the signal of interest by using only cosines with a period longer than a given threshold and therefore avoids the injection of artificial signals with periods shorter than this threshold. Teachey et al. (2018) also test polynomial detrending functions but report that this removes the possible exomoon signal. We choose to reimplement the CoFiAM algorithm as our primary detrending algorithm so as to remain as close as possible in our analysis to the work in Teachey et al. (2018). In our injection-retrieval test, we also use polynomials of second, third, and fourth-order for detrending. While low-order polynomials cannot generally fit the light curve as well as the series of cosines, the risk of injecting artificial signals may be reduced.

\subsubsection{Trigonometric detrending}

We implement the CoFiAM detrending algorithm as per the descriptions given by Kipping et al. (2013b) and Teachey et al. (2018). In the following, we refer to this reimplementation as trigonometric detrending as opposed to polynomial functions that we test as well (see Sect. 2.2.4.4).

The light curves around each transit are detrended independently. For each transit, we start by using the entire SAP flux of the corresponding quarter. We use the SAP flux instead of the PDCSAP flux to reproduce the methodology of Teachey et al. (2018) as closely as possible. The authors argue that the use of SAP flux avoids the injection of additional signals into the light curve that might have the shape of a moon signal. First, we remove outliers using a running median with a window length of $20 \mathrm{~h}$ and a threshold of three times the local standard deviation with the same window length. In order to achieve 


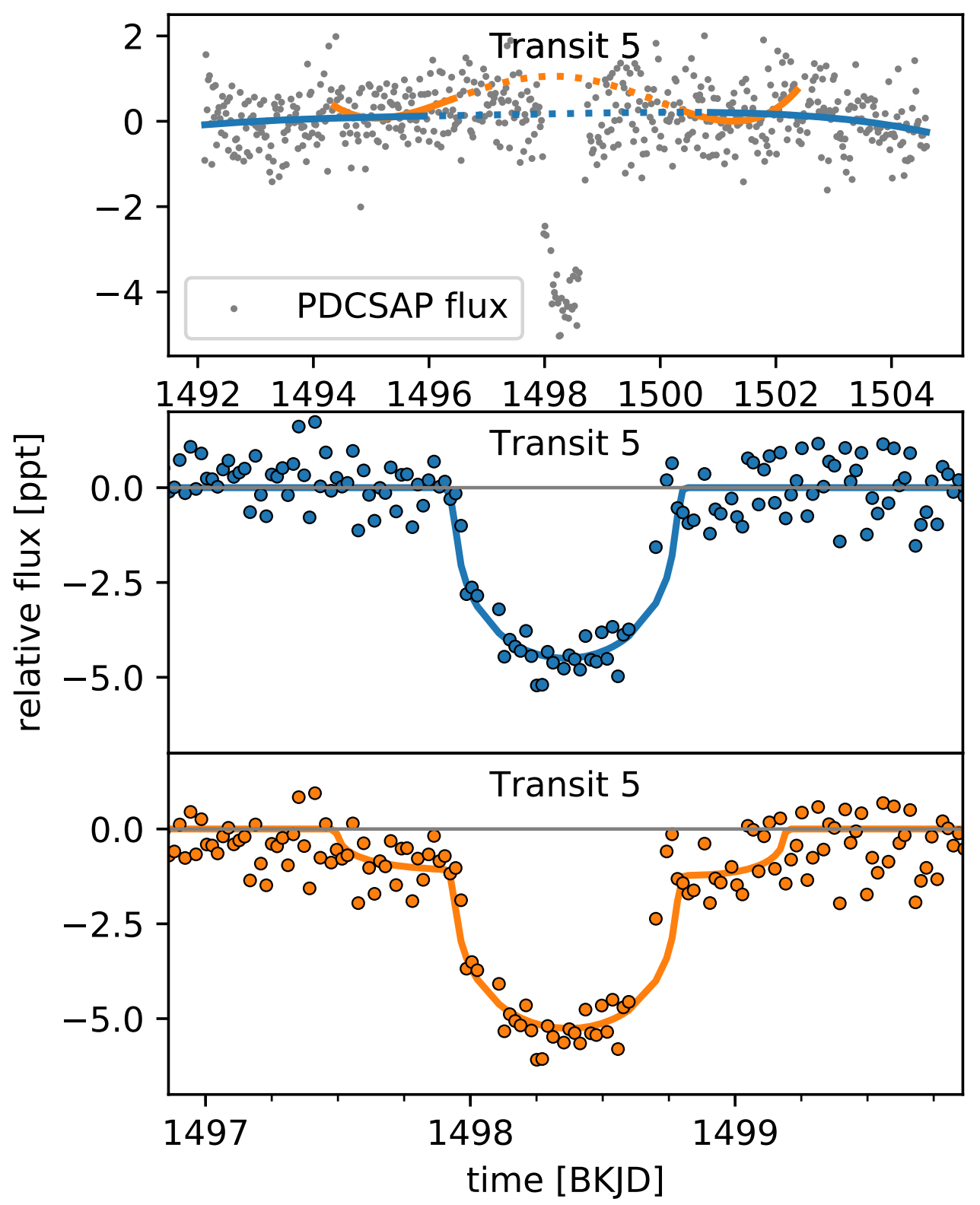

Figure 2.2: Example of how the detrending procedure alone can produce an exomoon-like transit signal around a planetary transit. We use 'transit 5' of Kepler-1625 b as an example. Top: Gray dots indicate the Kepler PDCSAP flux. The lines show a fourth-order polynomial fit for which we exclude $7.5 \mathrm{~d}$ (blue) or $4 \mathrm{~d}$ (orange) of data around the midpoint (dashed parts), respectively. Center: Dots show the detrended light curve derived from the blue polynomial fit in the top panel. The blue line illustrates a planet-only transit model. Bottom: Dots visualize the detrended light curve using the orange polynomial fit from the top panel. We note the additional moon-like transit feature caused by the overshooting of the orange polynomial in the top panel. The orange line shows a planet-moon transit model with moon parameters as in Table 2.1 (see Fig. 2.4 for transit dynamics). As an alternative interpretation, the blue detrending function filters out an actually existing moon signature while the orange detrending fit preserves the moon signal. 

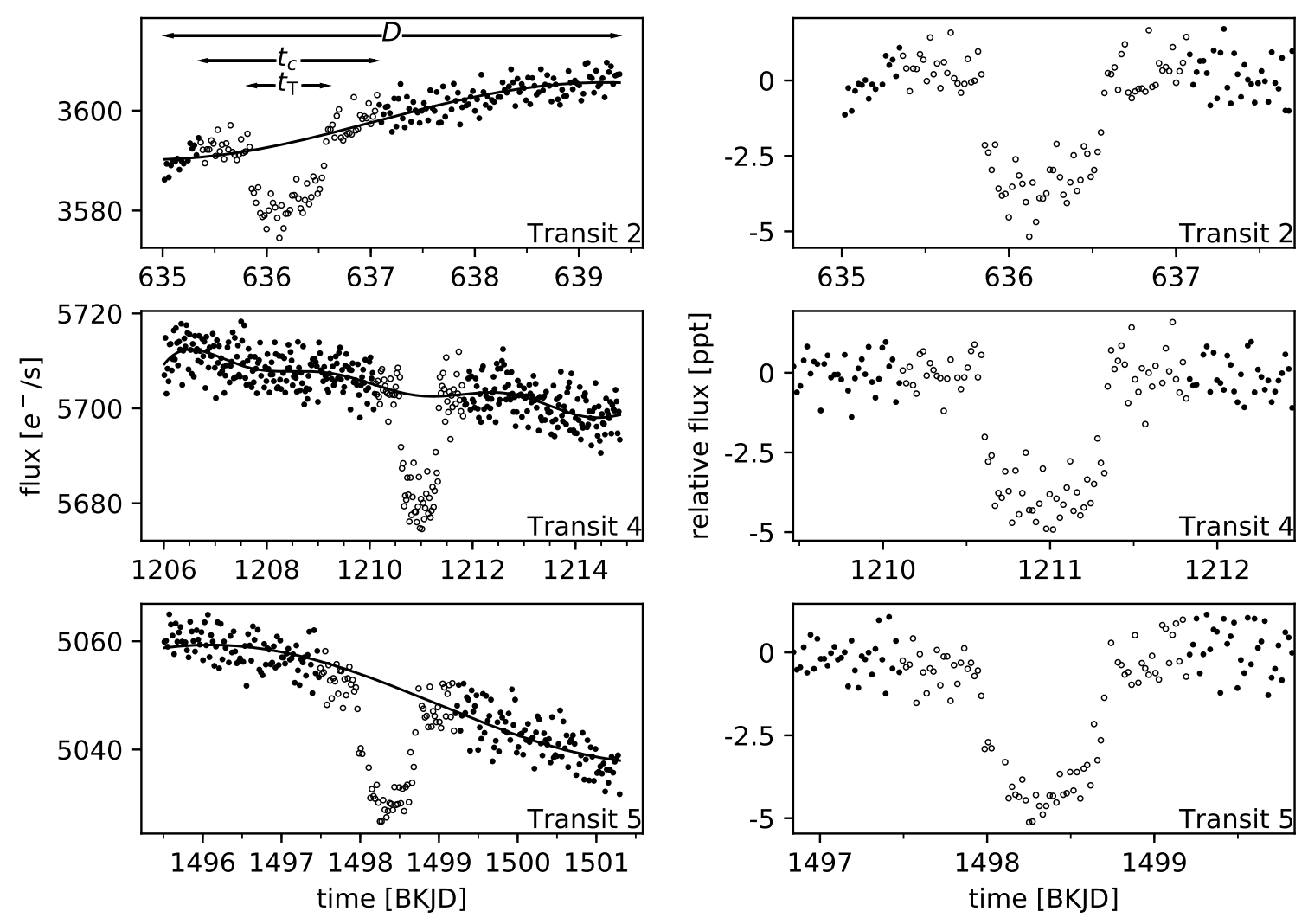

Figure 2.3: Left: Kepler SAP flux around the transits used for the trigonometric detrending, our reimplementation of the CoFiAM algorithm. The data points denoted by open circles around the transits are excluded from the detrending fit. The black line shows the resulting light curve trend without the transit. Right: Detrended transit light curves as calculated by the trigonometric detrending.

a fast convergence of our detrending and transit fitting procedures, we initially estimate the transit midpoints and durations by eye and identify data anomalies: for example, gaps and jumps (e.g. the jump $2 \mathrm{~d}$ prior to transit 2 and the gap $4 \mathrm{~d}$ after transit 4 , see Fig. 2.1).

Jumps in the light curve can have different origins. The jumps highlighted around transit 2 in Fig. 2.1 are caused by a reaction wheel zero crossing event; the jump $5 \mathrm{~d}$ after transit 4 is caused by a change in temperature after a break in the data collection. Following Teachey et al. (2018), who ignore data points beyond gaps and other anomalous events for detrending, we cut the light curve around any of the transits as soon as it encounters the first anomaly, leaving us with a light curve of a total duration $D$ around each transit (see top left panel in Fig. 2.3). In Sect.2.2.4.4, we investigate the effect of including data beyond gaps. The detrending is then applied in two passes, using the first pass to get accurate transit parameters. In particular, we determine the duration $\left(t_{\mathrm{T}}\right)$ between the start of the planetary transit ingress and the end of the transit egress (Seager and Mallén-Ornelas 2003) and the second pass to generate the detrended light curve.

First pass: Using the estimated transit midpoints and durations, we calculate the time window ( $t_{\mathrm{c}}$, see top left panel in Fig. 2.3) around a given transit midpoint to be cut from the detrending fit as $t_{c}=f_{t_{\mathrm{c}}} t_{\mathrm{T}}$, where the factor $f_{t_{\mathrm{c}}}$, relating the time cut around the transit to the transit duration, is an input parameter for the detrending algorithm. Specifically, $t_{\mathrm{c}}$ 
denotes the total length of time around the transit excluded from the detrending. We fit the detrending function,

$$
\mathcal{G}^{k}(t, \vec{a}, \vec{b})=a_{0}+\sum_{l=1}^{k} a_{l} \cos \left(l \frac{2 \pi}{2 D} t\right)+b_{l} \sin \left(l \frac{2 \pi}{2 D} t\right),
$$

to the light curve (excluding the region $t_{\mathrm{c}}$ around the transit) by minimizing the $\chi^{2}$ between the light curve and $\mathcal{G}^{k}(t, \vec{a}, \vec{b})$, where $\vec{a}=\left(a_{0}, a_{1}, \ldots, a_{k}\right)$ and $\vec{b}=\left(b_{1}, b_{2}, \ldots, b_{k}\right)$ are the free model parameters to be fitted. The parameter $k$ is a number between 1 and $k_{\max }=\operatorname{round}\left(2 D / t_{\mathrm{p}}\right)$, where $t_{\mathrm{p}}=f_{t_{\mathrm{p}}} t_{\mathrm{T}}$ is the timescale below which we want to preserve possible signals. $f_{t_{\mathrm{p}}}$ is an input parameter to the detrending algorithm. For each $k$, we divide the light curve by $\mathcal{G}^{k}$, giving us the detrended light curves $F^{k}$. We calculate the first-order autocorrelation according to the Durbin and Watson (1950) test statistic for each $F^{k}$ (excluding again the region around the transit). For each transit, we select the $F^{k}$ with the lowest autocorrelation $F_{\min }^{k}$ and combine these $F_{\min }^{k}$ around each transit into our detrended light curve $F$. We fit the planet-only transit model to the detrended light curve $F$ and compute the updated transit midpoints and duration $t_{\mathrm{T}}$.

Second pass: The second pass repeats the steps of the first pass, but using the updated transit midpoints and durations as input. The resulting detrended light curve $F$ is then used for our model fits with the ultimate goal being to assess whether or not an exomoon is a likely interpretation of the light curve signatures. We estimate the noise around each transit by taking the variance of $F$, excluding the transit region.

Figure 2.3 shows the detrending function as well as the detrended light curve for $f_{t_{c}}=2.2$ and $f_{t_{p}}=4.4$, corresponding to $t_{c}=1.6 \mathrm{~d}$ and $t_{p}=3.1 \mathrm{~d}$.

\subsubsection{Transit model}

We construct two transit models, one of which contains a planet only and one of which contains a planet with one moon. We denote the planet-only model as $\mathcal{M}_{0}$ (the index referring to the number of moons) and the planet-moon model as $\mathcal{M}_{1}$. We do not consider models with more than one moon.

\subsubsection{Planet-only model}

$\mathcal{M}_{0}$ assumes a circular orbit of the planet around its star. Given the period of that orbit $(P)$ and the ratio between stellar radius and the orbital semi-major axis $\left(R_{\star} / a\right)$, the skyprojected apparent distance to the star center relative to the stellar radius can be calculated as

$$
z=\sqrt{\left[\frac{a}{R_{\star}} \sin \left(\frac{2 \pi\left(t-t_{0}\right)}{P}\right)\right]^{2}+\left[b \cos \left(\frac{2 \pi\left(t-t_{0}\right)}{P}\right)\right]^{2}},
$$

where $b$ is the transit impact parameter and $t_{0}$ is the time of the transit midpoint. We use the python implementation of the Mandel and Agol (2002) analytic transit model by Ian Crossfield ${ }^{3}$ to calculate the transit light curve based on the planet-to-star radius ratio

\footnotetext{
${ }^{3}$ Available at http://www.astro.ucla.edu/ ianc/files as python.py.
} 


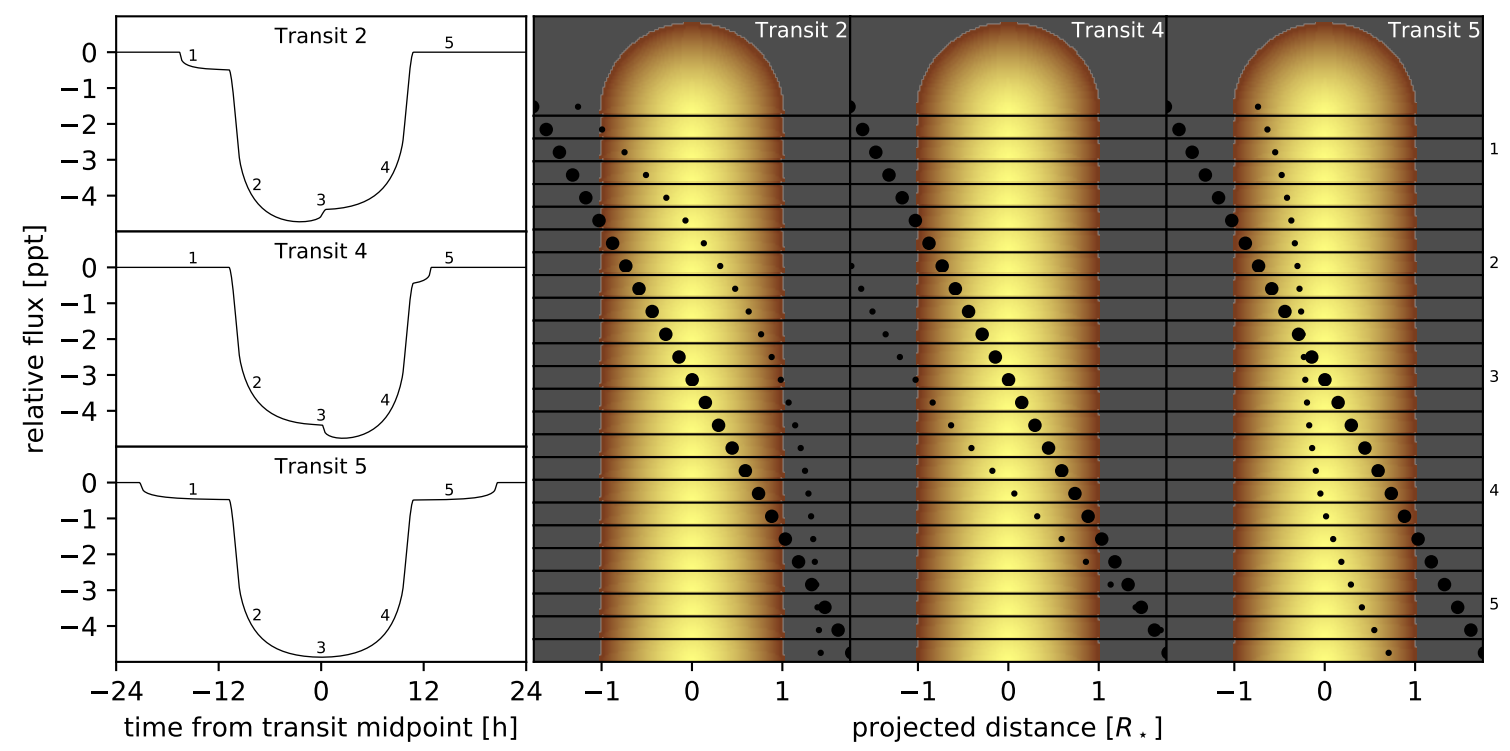

Figure 2.4: Left: Example of a simulated planet-moon transit light curve for transits 2, 4, and 5 using the nominal parameterization given in Table 2.1. The relative flux is the difference to the out-of-transit model flux and is given in parts per thousand (ppt). Right: Visualization of the orbital configurations during transits 2 (left column), 4 (center column), and 5 (right column). Labels 1-5 in the light curves refer to configurations 1-5 (see labels along the vertical axis). An animated version of this figure is available online.

$\left(r_{\mathrm{p}}=R_{\mathrm{p}} / R_{\star}\right)$ and based on a quadratic limb-darkening law parametrized by the limbdarkening parameters $q_{1}$ and $q_{2}$ as given in Kipping (2013). We call this model light curve with zero moons $\mathcal{F}_{0}$.

\subsubsection{Planet-moon model}

In our planet-moon model, we assume a circular orbit of the local planet-moon barycenter around the star with an orbital period $P_{\mathrm{B}}$, a semimajor axis $a_{\mathrm{B}}$, and a barycentric transit midpoint time $t_{0, \mathrm{~B}}$. The projected distance of the barycenter to the star center relative to the stellar radius is calculated in the same way as in Eq. 2.2. The planet and moon are assumed to be on circular orbits around their common center of mass with their relative distances to the barycenter determined by the ratio of their masses $M_{\mathrm{p}}$ and $M_{\mathrm{s}}$ to the total mass $M_{\mathrm{p}}+M_{\mathrm{s}}$. The individual orbits of both the planet and the moon are defined by the total distance between the two objects $a_{\mathrm{ps}}$, the planet mass $M_{\mathrm{p}}$, the moon mass $M_{\mathrm{s}}$ and by the time of the planet-moon conjunction $t_{0, \mathrm{~s}}$, that is, the time at which the moon is directly in front of the planet as seen from an observer on Earth.

This model is degenerate in terms of the sense of orbital motion of the moon. In other words, a given planet-moon transit light curve can be produced by both a prograde and a retrograde moon (Lewis and Fujii 2014, Heller and Albrecht 2014). We restrict ourselves to prograde moons. The planet mass is set to a nominal ten Jupiter masses, as suggested by Teachey et al. (2018) and in agreement with the estimates of Heller (2018a). This constraint simplifies the interpretation of the results substantially since the moon parameters are then unaffected by the planetary parameters. The moon mass is assumed 
Table 2.1: Nominal parameterization of the planet-moon model to reproduce the transit shape suggested by Teachey et al. (2018). The no-moon model uses the same parameter set (excluding the moon parameters), except that $R_{\star}$ and $a_{\mathrm{B}}$ are combined into a single parameter $R_{\star} / a_{\mathrm{B}}$.

\begin{tabular}{|c|c|c|}
\hline Parameter & Nominal Value & Description \\
\hline$r_{\mathrm{p}}$ & 0.06 & planet-to-star radius ratio \\
\hline$a_{\mathrm{B}}$ & $0.9 \mathrm{au}$ & $\begin{array}{l}\text { circumstellar semimajor axis of the } \\
\text { planet-moon barycenter }\end{array}$ \\
\hline$b$ & 0.1 & planetary transit impact parameter \\
\hline$t_{0, \mathrm{~B}}$ & $61.51 \mathrm{~d}$ & $\begin{array}{l}\text { transit midpoint of the planet-moon } \\
\text { barycenter }\end{array}$ \\
\hline$P_{\mathrm{B}}$ & $287.35 \mathrm{~d}$ & $\begin{array}{l}\text { circumstellar orbital period of the } \\
\text { planet-moon barycenter }\end{array}$ \\
\hline$R_{\star}$ & $1.8 R_{\odot}$ & stellar radius \\
\hline$q_{1}$ & 0.6 & first limb darkening coefficient \\
\hline$q_{2}$ & 0.2 & second limb darkening coefficient \\
\hline$r_{\mathrm{s}}$ & 0.02 & moon-to-star radius ratio \\
\hline$a_{\mathrm{s}}$ & $1871 R_{\mathrm{J}}$ & $\begin{array}{l}\text { orbital semimajor axis of the planet- } \\
\text { moon binary }\end{array}$ \\
\hline$t_{0, \mathrm{~s}}$ & $1.86 \mathrm{~d}$ & time of planet-moon conjunction \\
\hline
\end{tabular}

to be much smaller than that of the planet. In fact, for a roughly Neptune-mass moon around a planet of ten Jupiter masses, we expect a TTV amplitude of 3 to 4 minutes and a TDV amplitude of 6 to 7 minutes, roughly speaking. Therefore, we simplify our model and set $M_{\mathrm{s}}=0$, which means that $a_{\mathrm{ps}}$ is equal to the distance between the moon and the planet-moon barycenter, $a_{\mathrm{s}}$. The moon is assumed to have a coplanar orbit around the planet and, thus, to have the same transit impact parameter as the planet.

With these assumptions, the projected distance of the planet center to the star center relative to the stellar radius $z_{\mathrm{p}}$ is equal to that of the barycenter $z_{\mathrm{B}}$. The projected distance of the moon center to the star center relative to the stellar radius $z_{\mathrm{s}}$ is given by

$$
\begin{aligned}
z_{\mathrm{s}}^{2} & =\left[\frac{a_{\mathrm{B}}}{R_{\star}} \sin \left(\frac{2 \pi\left(t-t_{0, \mathrm{~B}}\right)}{P_{\mathrm{B}}}\right)+\frac{a_{\mathrm{ps}}}{R_{\star}} \sin \left(\frac{2 \pi\left(t-t_{0, \mathrm{~s}}\right)}{P_{\mathrm{s}}}\right)\right]^{2} \\
& +\left[b \cos \left(\frac{2 \pi\left(t-t_{0, \mathrm{~B}}\right)}{P_{\mathrm{B}}}\right)\right]^{2},
\end{aligned}
$$

where $P_{\mathrm{s}}$ is the orbital period of the moon calculated from the fixed masses and $a_{\mathrm{ps}}$.

We calculate the transit light curves of both bodies and combine them into the total model light curve, which we call $\mathcal{F}_{1}$. We use the limb-darkening parameter transformation from Kipping (2013). For computational efficiency, we do not consider planet-moon occultations. For the planet-moon system of interest, occultations would only occur during about half of the transits (assuming a random moon phase) even if the moon orbital 
plane were perfectly parallel to the line of sight. Such an occultation would take about $1.5 \mathrm{~h}$ and would only affect $5-10 \%$ of the total moon signal duration.

In Table 2.1 we give an overview of our nominal parameterization of the planet-moon model. In Fig. 2.4 we show the orbital dynamics of the planet and moon during transits 2, 4, and 5 using the nominal parameters in Table 2.1. This nominal parameterization was chosen to generate a model light curve that is reasonably close to the preferred model light curve found in Teachey et al. (2018), but it does not represent our most likely model fit to the data.

\subsubsection{Finding the posterior probability distribution}

We use the Markov Chain Monte Carlo (MCMC) implementation Emcee (Foreman-Mackey et al. 2013) to estimate the posterior probability distribution of the parameters for model $\mathcal{M}_{(i)}\left(\mathcal{M}_{0}\right.$ or $\left.\mathcal{M}_{1}\right)$. For this purpose, we need to formulate the probability density of a light curve as well as the prior of the parameters.

All three transits taken together, we have a total of $N$ detrended flux measurements (see Sect. 2.2.1). Given a set of parameters $\vec{\theta}$, model $\mathcal{M}_{i}$ produces a model light curve $\mathcal{F}_{i}(t, \vec{\theta})$. We assume that the noise is uncorrelated (see Appendix 2.B) and Gaussian with a standard deviation $\sigma_{j}$ at time $t_{j}$. This simplifies the joint probability density to a product of the individual probabilities. The joint probability density function of the detrended flux $F(t)$ is given by

$$
p\left(F \mid \vec{\theta}, \mathcal{M}_{i}\right)=\prod_{j=1}^{N} \frac{1}{\sqrt{2 \pi \sigma_{j}^{2}}} \exp \left(-\frac{\left(F\left(t_{j}\right)-\mathcal{F}_{i}\left(t_{j}, \vec{\theta}\right)\right)^{2}}{2 \sigma_{j}^{2}}\right)
$$

The noise dispersion $\sigma_{j}$ has a fixed value for each transit.

Table 2.2 shows the parameter ranges that we explore. A prior is placed on the stellar mass according to the mass of $1.079_{-0.138}^{+0.100} M_{\odot}$ determined by Mathur et al. (2017). The stellar mass for a given parameter set is determined from the system's total mass using $P_{\mathrm{B}}$ and $a_{\mathrm{B}}$ and subtracting the fixed planet mass of ten Jupiter masses.

A total of 100 walkers are initiated with randomly chosen parameters close to the estimated transit parameters. For the sake of fast computational performance, the walkers are initially separated into groups of 16 for the planet-only model and 24 for the planetmoon model (twice the number of parameters plus 2, respectively), temporarily adding walkers to fill the last group. To transform the initially flat distribution of walkers into a distribution according to the likelihood function, the walkers have to go through a socalled burn-in phase, the resulting model fits of which are discarded. We chose a burn-in phase for the walkers of 500 steps in both groups. Afterwards, we discard the temporarily added walkers, merge the walkers back together, and perform a second burn-in phase of 2200 steps with a length determined by visual inspection. Finally, we initiate the main MCMC run with a total of 8000 steps.

We run the MCMC code on the detrended light curve using both the planet-only and the planet-moon models. 
Table 2.2: Parameter ranges explored with our planet-moon model. The ranges of the no-moon model parameters are the same for the shared parameter and are propagated to the derived parameter $R_{\star} / a$.

\begin{tabular}{ccccc}
\hline \hline Min. Value & & Parameter & & Max. Value \\
\hline 0 & $\leq$ & $r_{\mathrm{p}}$ & $\leq$ & 0.1 \\
$0.2 \mathrm{au}$ & $\leq$ & $a_{\mathrm{B}}$ & $\leq$ & $2 \mathrm{au}$ \\
0 & $\leq$ & $b$ & $\leq$ & 1 \\
$-P_{\mathrm{B}} / 2$ & $\leq$ & $t_{0, \mathrm{~B}}$ & $\leq$ & $P_{\mathrm{B}} / 2$ \\
$270 \mathrm{~d}$ & $\leq$ & $P_{\mathrm{B}}$ & $\leq$ & $300 \mathrm{~d}$ \\
\hline 0 & $\leq$ & $R_{\star}$ & $\leq$ & $4.3 R_{\odot}$ \\
0 & $\leq$ & $q_{1}$ & $\leq$ & 1 \\
0 & $\leq$ & $q_{1}$ & $\leq$ & 1 \\
\hline 0 & $\leq$ & $r_{\mathrm{s}}$ & $\leq$ & $r_{\mathrm{p}}$ \\
0 & $\leq$ & $a_{\mathrm{s}}$ & $\leq$ & $R_{\mathrm{Hill}} / 2$ \\
$-P_{\mathrm{s}} / 2$ & $\leq$ & $t_{0, \mathrm{~s}}$ & $\leq$ & $P_{\mathrm{s}} / 2$
\end{tabular}

\subsubsection{Model selection}

We use the Bayesian information criterion (BIC) to evaluate how well a model describes the observations in relation to the number of model parameters and data points. The BIC of a given model $\mathcal{M}_{i}$ with $m_{i}$ parameters is defined by Schwarz (1978) as

$$
\operatorname{BIC}\left(\mathcal{M}_{i} \mid F\right)=m_{i} \ln N-2 \ln p\left(F \mid \vec{\theta}_{\max }, \mathcal{M}_{i}\right),
$$

where $\vec{\theta}_{\max }$ is the set of parameters that maximizes the probability density function $p\left(F \mid \vec{\theta}, \mathcal{M}_{i}\right)$ for a given the light curve $F$ and model $\mathcal{M}_{i}$.

The difference of the BICs between two models gives an indication as to which model is more likely. In particular, $\Delta \mathrm{BIC}\left(\mathcal{M}_{1}, \mathcal{M}_{0}\right) \equiv \operatorname{BIC}\left(\mathcal{M}_{1}\right)-\operatorname{BIC}\left(\mathcal{M}_{0}\right)<0$ if model $\mathcal{M}_{1}$ is more likely. We consider $\triangle \mathrm{BIC}<6$ (or $\triangle \mathrm{BIC}>6$ ) as strong evidence in favor of (or against) model $\mathcal{M}_{1}$ (see, e.g., Kass and Raftery 1995).

The best-fitting set of parameters derived from our MCMC runs $\left(\vec{\theta}_{\max }\right)$ is then used to calculate $\triangle \operatorname{BIC}\left(\mathcal{M}_{1}, \mathcal{M}_{0}\right)$. For our calculations, we only use those parts of the light curve around the transits that could potentially be affected by a moon $(3.25 \mathrm{~d}$ on each side of the transits, determined by the Hill radius $R_{\text {Hill }}$ and the orbital velocity of the planet-moon barycenter; see Appendix 2.A.).

\subsubsection{Injection-retrieval test}

In order to estimate the likelihood of an exomoon feature to be due to either a real moon or due to noise, we perform several injection-retrieval experiments. One of us $(\mathrm{MH})$ injected two cases of transits into the out-of-transit parts of the original PDCSAP Kepler flux. In one case, a sequence of three planet-only transits (similar to the sequence of real transits 
2, 4, and 5) was injected, where the planet was chosen to have a radius of $11 R_{\oplus}$. In another case, a sequence of three transits of a planet-with-moon system, with properties similar to the proposed Jupiter-Neptune system, was injected. Author KR then applied the Baysian framework described above in order to evaluate the planet-only versus the planet-with-moon hypotheses, and in order to characterize the planet and (if present) its moon. As an important trait of our experiment, the author KR did not know which of the light curves contained only a planet and which contained also a moon.

\subsubsection{Transit injections into light curves}

For the injection part, we use PyOSE (Heller et al. 2016a b) to create synthetic planet and moon ensemble transits. This code numerically integrates the non-occulted areas of the stellar disk to calculate the instantaneous flux of the star, which makes it a computationally slow procedure. We therefore use the analytical model described Sect. 2.2.2 for the retrieval part. In our model, the moon's orbit is defined by its eccentricity $\left(e_{\mathrm{s}}\right.$, fixed at $0), a_{\mathrm{s}}$, its orbital inclination with respect to the circumstellar orbit $\left(i_{\mathrm{s}}\right.$, fixed at $\left.90^{\circ}\right)$, the longitude of the ascending node, the argument of the periapsis, and the planetary impact parameter ( $b$, fixed at zero). Due to the small TTV and TDV amplitudes compared to the 29.4 min exposure of the Kepler long cadence data, we neglect the planet's motion around the planet/moon barycenter (although PyOSE can model this dynamical effect as well) and assume that the moon orbits the center of the planet.

Our numerical code creates a spherical limb-darkened star on a two-dimensional grid of floating-point values. The sky-projected shapes of both the planet and the moon are modeled as black circles. The spatial resolution of the simulation is chosen to be a few million pixels so that the resulting light curve has a numerical error of $<1 \mathrm{ppm}$, which is negligible compared to the $\approx 700 \mathrm{ppm}$ noise level of the Kepler light curve. The initial temporal resolution of our model is equivalent to 1000 steps per planetary transit duration, which we then downsample to the observed 29.4 min cadence. The creation of one such light curve of a planet with a moon takes about one minute on a modern desktop computer.

We create a set of 100 such transit simulations of the planet-moon ensemble, where the two bodies move consistently during and between transits. All orbits are modeled to be co-planar and mutual planet-moon occultations are also included. For each transit sequence, the initial orbital phase of the planet-moon system is chosen randomly.

With $P_{\mathrm{B}}=287.378949 \mathrm{~d}$ and $P_{\mathrm{s}}=2.20833 \mathrm{~d}$, the moon advances by $\approx 0.13$ in phase between each subsequent planetary transit $\left(P_{\mathrm{B}} / P_{\mathrm{s}} \approx 130.13\right)$. During a planetary transit, the moon advances by $\approx 0.36 \mathrm{rad}$ in phase (the planetary transit duration is $0.7869 \pm$ $0.0084 \mathrm{~d})$.

We also create a set of 100 such transits that only have a transiting planet without a moon. In these cases, the planetary radius was increased slightly to match the average transit depth of planet and moon.

\subsubsection{Testing the model-selection algorithm on synthetic light curves with white noise only}

As a first validation of our injection-retrieval experiment and our implementation of the Bayesian statistical framework, we generate a new set of white noise light curves to test 
planet-only system injected

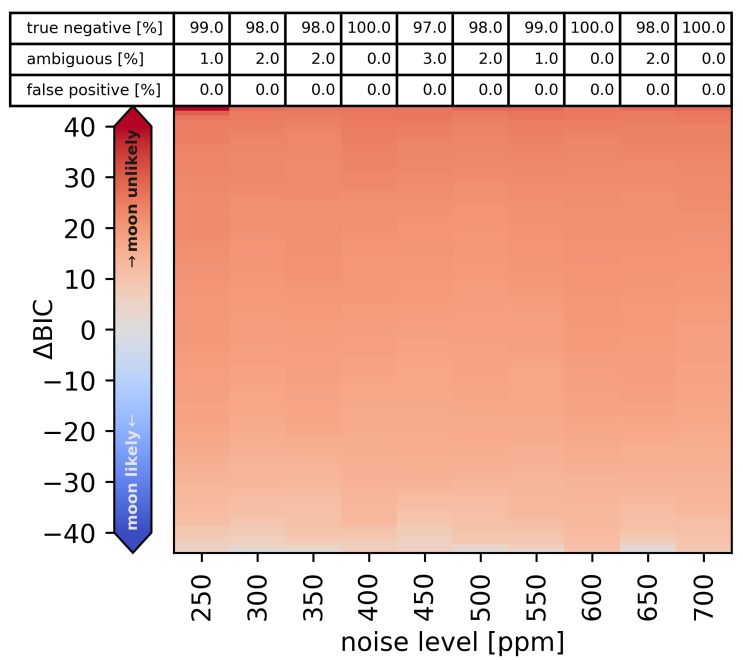

planet+moon system injected

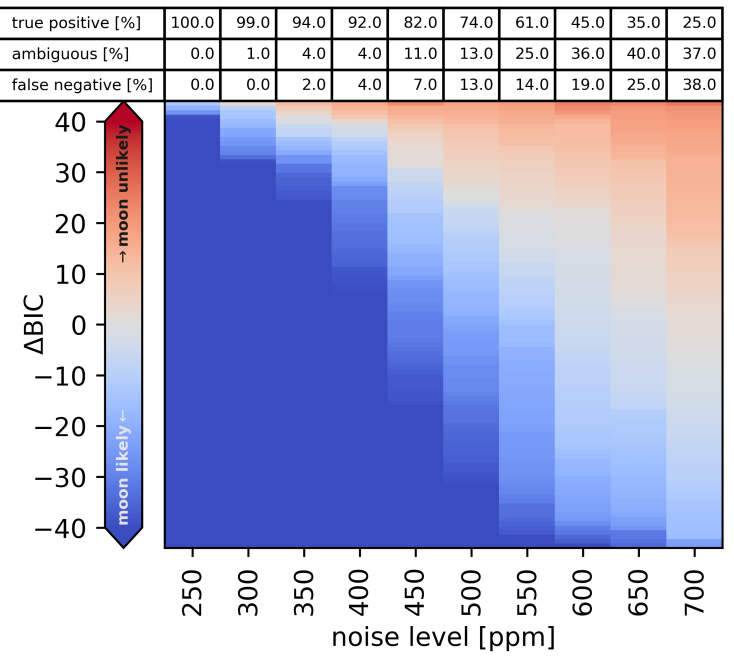

Figure 2.5: Difference between the BIC of the planet-moon model and the no-moon model for 2000 artificial white noise light curves at different noise levels, injected with simulated transits. On the left $(100 \times 10$ light curves), a planet and moon transit was injected, on the right $(100 \times 10$ light curves), only the planet. Each light curve consists of three consecutive transits. Each column is sorted by the $\triangle \mathrm{BIC}$. The $\triangle \mathrm{BIC}$ threshold, over which a planetmoon or planet-only system is clearly preferred is \pm 6 with the state of systems with a $\triangle \mathrm{BIC}$ between those values considered to be ambiguous.

only the model comparison part of our pipeline without any effects that could possibly arise from imperfect detrending. Any effects that we would see in our experiments with the real Kepler-1625 light curve but not in the synthetic light curves with noise only could then be attributed to the imperfect detrending of the time-correlated (red) noise.

The author MH generated 200 synthetic light curves with ten different levels of white noise, respectively, ranging from root mean squares of $250 \mathrm{ppm}$ to $700 \mathrm{ppm}$ in steps of $50 \mathrm{ppm}$. This results in a total of 2000 synthetic light curves. The method described in Sect. 2.2.4.1 was used to inject three transits of a planet only into 100 light curves per noise level and three transits of a planet with a moon into the remaining 100 light curves per noise level. The initial orbital phases were randomly chosen and are different from the ones used to generate the light curves in Sect.2.2.4.3. The author MH delivered these light curves to the author KR without revealing their specific contents. The author KR then ran our model selection algorithm to find the $\triangle \mathrm{BIC}$ for each of the 2000 systems. After the $\triangle \mathrm{BICs}$ were found, $\mathrm{MH}$ revealed the planet-only or the planet-moon nature of each light curve.

Figure 2.5 shows the resulting $\triangle \mathrm{BICs}$ for each of the 2000 light curves, separated into the planet-only (left panel) and planet-moon injected systems (right panel) and sorted by the respective white noise level (along the abscissa). Each vertical column contains 100 light curves, respectively. For a noise level of $250 \mathrm{ppm}$, for example, our algorithm finds no false positive moons in the planet-only data, that is, no system with a $\triangle \mathrm{BIC}<-6$, while 1 case remains ambiguous $(-6<\Delta \mathrm{BIC}<6)$ and the other 99 cases are correctly identified as containing no moons. In the case of an injected planet-moon system instead, 
planet-only system injected
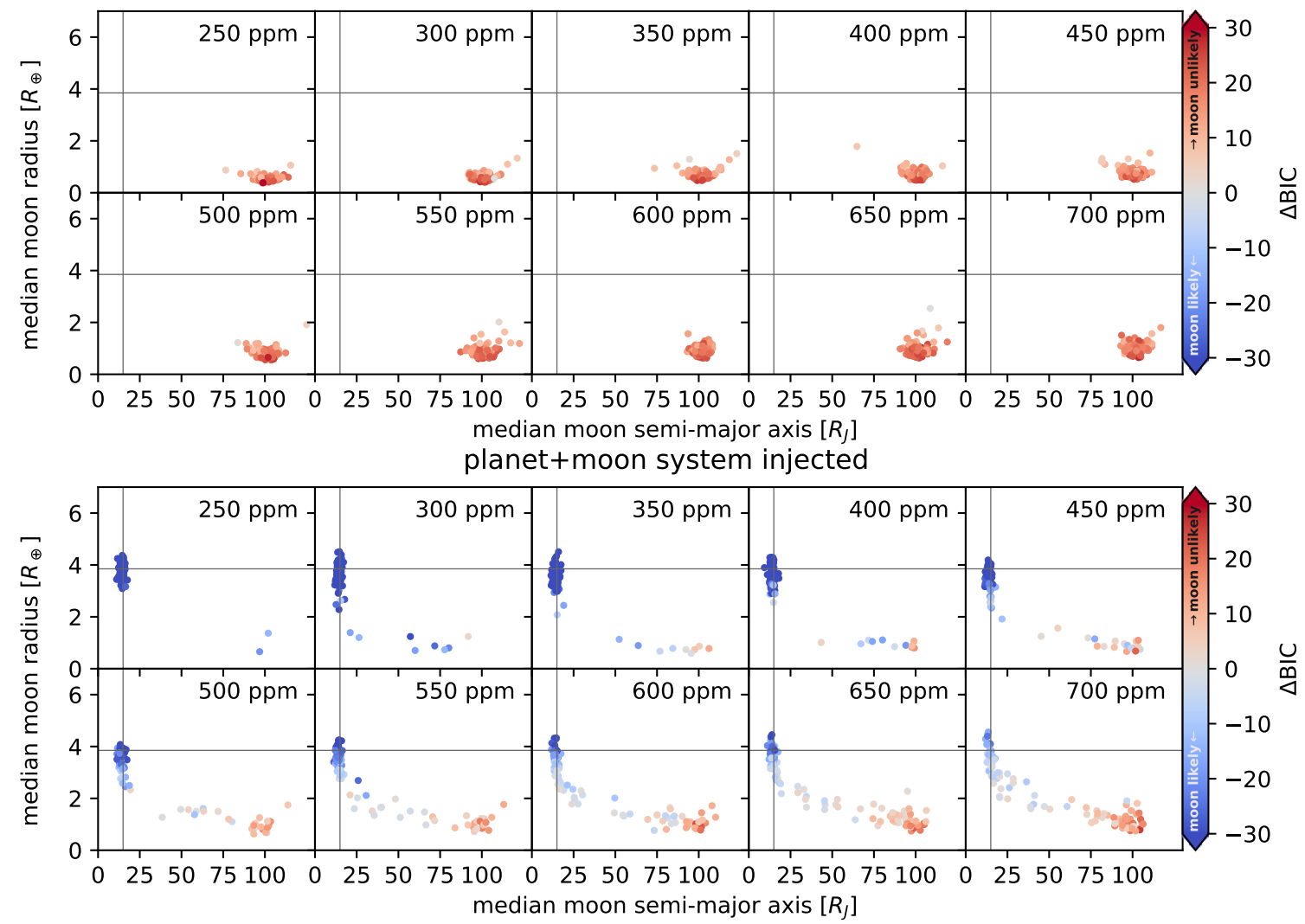

Figure 2.6: Distribution of the median likelihood $R_{\mathrm{S}}$ and $a_{\mathrm{s}}$ for all the runs for the different noise levels, with the runs injecting planet and moon on the top and runs injecting only a planet in the bottom. The $\triangle \mathrm{BIC}$ of the planet-moon model compared to the no-moon model for all runs is indicated by the color. Generally, runs with a low $\triangle \mathrm{BIC}$ (indicating the presence of a moon) also are in the vicinity of the injected parameter.

the algorithm correctly retrieves the moon in $100 \%$ of the synthetic light curves, that is, $\Delta \mathrm{BIC}<-6$ for all systems.

More generally, for the simulated planet-only systems, the false positive rate is $0 \%$ throughout all noise levels. Occasionally a system is flagged as ambiguous, but overall the algorithm consistently classifies planet-only systems correctly as having no moon. Referring to the injected planet-moon system (right panel), our false negative rate rises steadily with increasing noise level. In fact, it reaches parity with the true positive rate between about $650 \mathrm{ppm}$ and $700 \mathrm{ppm}$.

In Fig. 2.6, we present $a_{\mathrm{s}}$ and $R_{\mathrm{s}}$ for each of the maximum-likelihood fits shown in Fig. 2.5. Each panel in Fig. 2.6 refers to one white noise level, that is, to one column in Fig. 2.5 of either the planet-only or the planet-moon injected system. In the case of an injected planet only (upper panels), the most likely values of $a_{\mathrm{s}}$ are distributed almost randomly over the range of values that we explore. On the other hand, $R_{\mathrm{S}}$ is constrained to a small range from about $1.5 R_{\oplus}$ at $250 \mathrm{ppm}$ to roughly $3 R_{\oplus}$ at $700 \mathrm{ppm}$ with the standard variation naturally increasing with the noise level.

The lower part of Fig. 2.6 shows the outcome of our planet-moon injection-retrievals from the synthetic light curves with white noise only. The correct parameters are generally 
recovered at all noise levels. In fact, we either recover the moon with a similar radius and orbital separation as the injection values (symbolized by blue points) or we find the moon to have very different radius and orbit while also rejecting the hypothesis of its presence in the first place (symbolized by red points). The distribution of these false negatives in the $a_{\mathrm{s}}-R_{\mathrm{s}}$ plane resembles the distribution of the true negatives in the corresponding no-moon cases. The ambiguous runs with a $\triangle \mathrm{BIC}$ around zero still mostly recover the injected moon parameters. This is especially clear for the $700 \mathrm{ppm}$ level, with $50 \%$ more ambiguous runs than true positives, where most of the runs still recover the injected parameters.

\subsubsection{Transit injection into real out-of-transit data}

We inject synthetic transits into the Kepler-1625 PDCSAP data prior to our own detrending (see Sect. 2.2.4.1). We use the PDCSAP flux instead of SAP flux because (1) it was easier for us to automate the anomaly detection and (2) PDCSAP flux has been cleaned of common systematics. Since the PDC pipeline removes many of the jumps in the data, we can focus on a single type of anomaly, that is, gaps. Gaps are relatively easy to detect in an automated way, removing the requirement of visual inspection of each light curve. For the injection, we select out-of-transit parts of the Kepler-1625 light curve that have at least $50 \mathrm{~d}$ of mostly uninterrupted data $(25 \mathrm{~d}$ to both sides of the designated time of transit injection), but accept the presence of occasional gaps with durations of up to several days during the injection process.

The set of 200 synthetic light curves was provided by MH to KR for blind retrieval without any disclosure as to which of the sequences have a moon. The time of mid-transit was communicated with a precision of 0.1 days to avoid the requirement of a pre-stage transit search. This is justified because the original transits of Kepler-1625 b have already been detected, and the transits are visible by eye and do not necessarily need computerbased searches. We provide the 200 datasets to the community for reproducibility 4 and encourage further blind retrievals.

\subsubsection{Detrending of the transit-injected light curves}

The detrending procedure for our injection-retrieval experiment differs from the one used to detrend the original light curve around the Kepler-1625 b transits (see Sect. 2.2.1) in two respects.

First, we test the effect of the detrending function. In addition to the trigonometric function, we detrend the light curve by polynomials of second, third, and fourth order.

In addition, we test if the inclusion or exclusion of data beyond any gaps in the light curve affects the detrending. In one variation of our detrending procedure, we use the entire $\pm 25 \mathrm{~d}$ of data (excluding any data within $t_{\mathrm{c}}$ ) around a transit midpoint. In another variation, we restrict the detrending to the data up to the nearest gap (if present) on both sides of the transit.

To avoid the requirement of time-consuming visual inspections of each light curve, we construct an automatic rule to determine the presence of gaps, which are the most disruptive kind of artifact to our detrending procedure. We define a gap as an interruption

\footnotetext{
${ }^{4}$ Available on Zenodo, [10.5281/zenodo.1202034], Hippke (2018)
} 
Table 2.3: Definition of the detrending identifiers in relation to the respective detrending functions that we explored in our transit injection-retrieval experiment of the Kepler-1625 data. We define a gap as any empty parts in the light curve that show more than $12 \mathrm{~h}$ between consecutive data points. The trigonometric function refers to our reimplementation of the CoFiAM algorithm. P2 to P4 refer to polynomials of second to fourth order. T refers to our trigonometric detrending. $\mathrm{G}$ stands for the inclusion of data beyond gaps, $\mathrm{N}$ stands for the exclusion of data beyond gaps.

\begin{tabular}{ccc}
\hline \hline Identifier & Detrending Function & Reject Data Beyond Gap? \\
\hline P2/G & 2nd order polynomial & yes \\
P2/N & 2nd order polynomial & no \\
P3/G & 3rd order polynomial & yes \\
P3/N & 3rd order polynomial & no \\
P4/G & 4th order polynomial & yes \\
P4/N & 4th order polynomial & no \\
T/G & trigonometric & yes \\
T/N & trigonometric & no
\end{tabular}

of the data of more than half a day. Whenever we do detect a gap, we cut another $12 \mathrm{~h}$ at both the beginning and the end of the gap, since our visual inspection of the data showed that many gaps are preceded or followed by anomalous trends (see e.g. the gap $4 \mathrm{~d}$ after transit 4 in Fig. 2.1).

We ignore any data points within $t_{\mathrm{c}}$ around the transit midpoint (see Sect. 2.2.1). If a gap starts within an interval $\left[t_{\mathrm{c}} / 2, t_{\mathrm{c}} / 2+12 \mathrm{~h}\right]$ around the transit midpoint, then we lift our constraint of dismissing a $12 \mathrm{~h}$ interval around gaps and use all the data within $\left[t_{\mathrm{c}} / 2, t_{\mathrm{c}} / 2+12 \mathrm{~h}\right]$ plus any data up to $12 \mathrm{~h}$ around the next gap.

If all these cuts result in no data points for the detrending procedure to one side of one of the three transits in a sequence, then we ignore the entire sequence for our injectionretrieval experiment. This is the case for 40 out of the 200 artificially injected light curves. This high loss rate of our experimental data is a natural outcome of the gap distribution in the original Kepler-1625 light curve. We exclude these 40 light curves for all variations of the detrending procedure that we investigate. All things combined, these constraints produce synthetic light curves with gap characteristics similar to the original Kepler-1625 b transits (see Fig. 2.3), that is, we allow the simulation of light curves with gaps close to but not ranging into the transits. The four detrending functions and our two ways of treating gaps yield a total of eight different detrending methods that we investigate (see Table 2.3). 


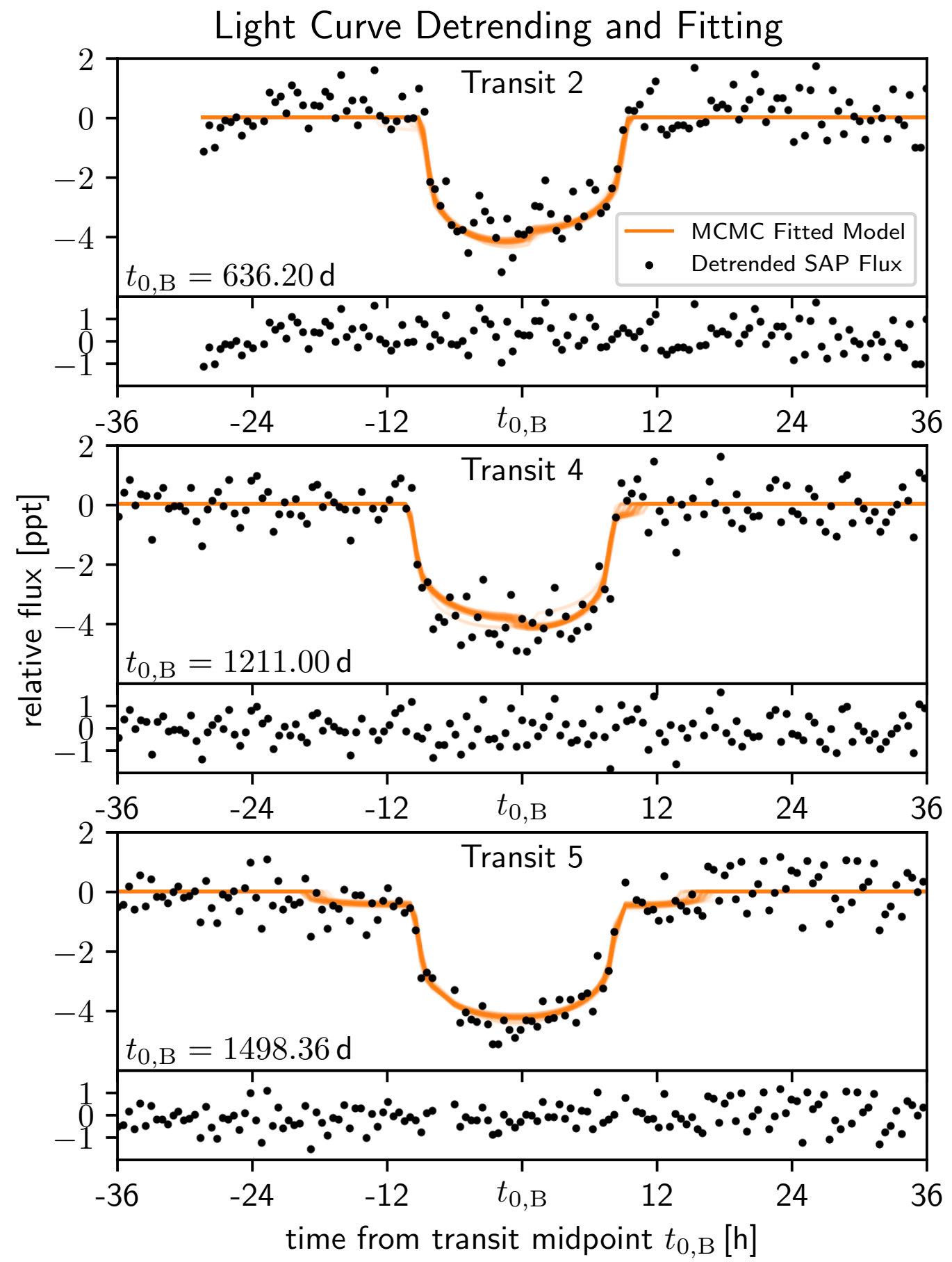

Figure 2.7: The observed second, fourth, and fifth transits of Kepler 1625 b. Black dots refer to our detrended light curve from the trigonometric detrending procedure, and orange curves are the model light curves generated using the 100 best fitting parameter sets of the MCMC run. The $\triangle \mathrm{BIC}$, calculated from the most likely parameters, is -4.954 . 


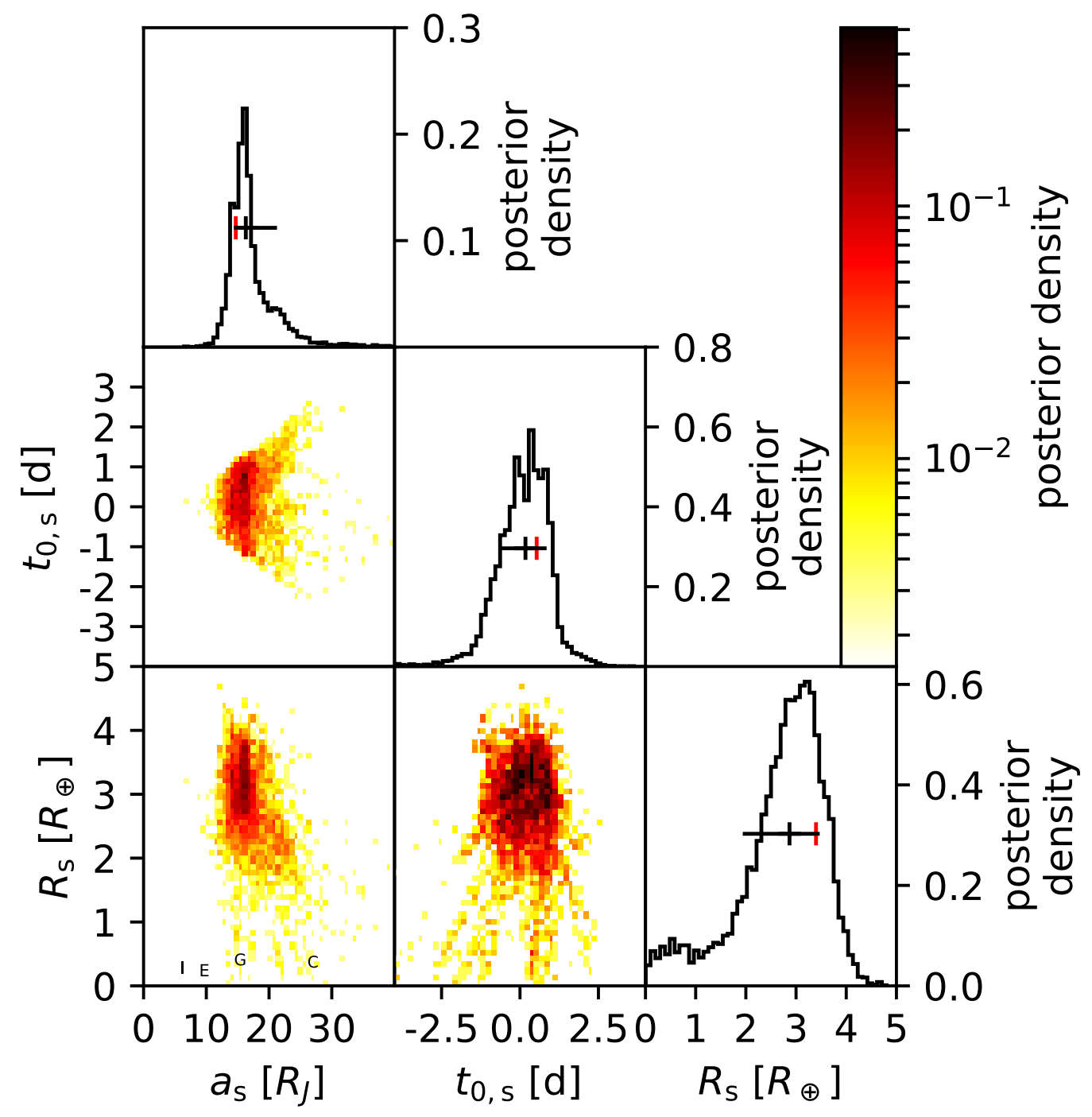

Figure 2.8: Posterior probability distribution of the moon parameters generated by the MCMC algorithm for the light curve detrended by the trigonometric detrending. The black vertical lines show the median of the posterior distribution, the black horizontal lines indicate the $1 \sigma$ range around the median. The red vertical lines show the point of maximum likelihood. The locations of the Galilean moons are included in the lower-left panel for comparison. 


\subsection{Results}

\subsubsection{Analysis of the original Kepler-1625 $b$ transits}

Our first result is a reproduction of a detrended transit light curve of Kepler-1625 b that has the same morphology and moon characterization as the one proposed by Teachey et al. (2018) and that has a negative $\triangle \mathrm{BIC}$. We explore the variation of the free parameters of our trigonometric detrending procedure, $f_{t_{p}}$ and $f_{t_{c}}$, and identify such a detrended light curve for $f_{t_{p}}=4.4$ and $f_{t_{c}}=2.2$. Figure 2.7 shows the resulting light curve.

In Fig. 2.8, we show the results of our MCMC analysis of this particular light curve, which yields a moon with $a_{\mathrm{s}}=16.3_{-1.9}^{+5.0} R_{\mathrm{J}}$ and $R_{\mathrm{s}}=2.87_{-0.94}^{+0.61} R_{\oplus}$. While both the moon radius and semimajor axis are well constrained, the distribution of the initial planet-moon orbital conjunction $\left(t_{0, \mathrm{~s}}\right)$ fills out almost the entire allowed parameter range from $-1 / 2 P_{\mathrm{s}}$ to $+1 / 2 P_{\mathrm{s}}$. The planetary radius is $0.863_{-0.051}^{+0.072} R_{\mathrm{J}}$, the stellar radius is $R_{\star}=1.57_{-0.09}^{+0.11} R_{\odot}$, and the density is $\rho_{\star}=0.26_{-0.05}^{+0.04} \rho_{\odot}$.

The point of maximum likelihood in the resulting MCMC distribution is at $a_{\mathrm{s}}=$ $14.7 R_{\mathrm{J}}, R_{\mathrm{S}}=3.4 R_{\oplus}, R_{\star}=1.57 R_{\odot}, \rho_{\star}=0.23 \rho_{\odot}$ and $R_{\mathrm{p}}=8.63 R_{\mathrm{J}}$. The $\Delta \mathrm{BIC}\left(\mathcal{M}_{1}, \mathcal{M}_{0}\right)$ we found is -4.954 , indicating moderate evidence in favor of an exomoon being in the light curve.

\subsubsection{Injection-retrieval experiment}
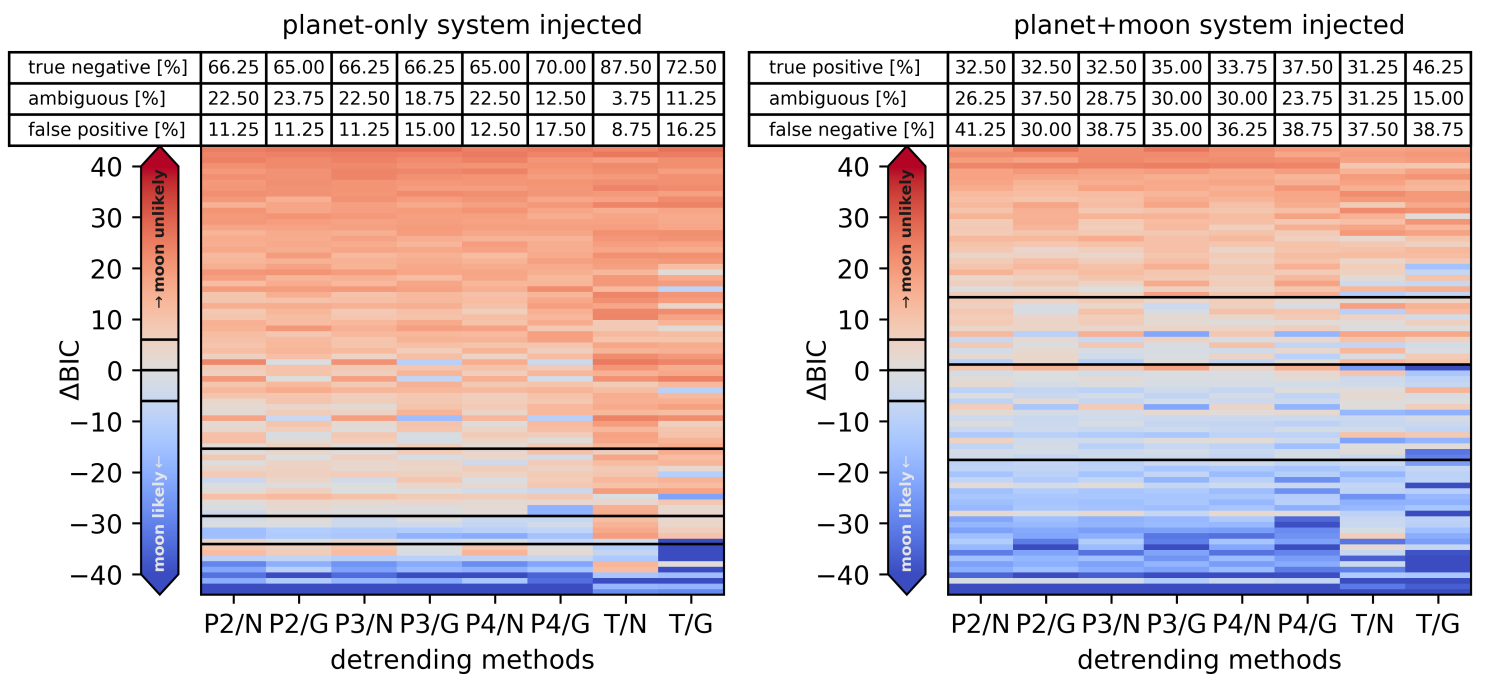

Figure 2.9: Difference between the BIC of the planet-moon model and the no-moon model using different detrending methods for 160 light curves, generated using the PDCSAP flux of Kepler 1625, injected with three simulated transits. On the left (80 light curves), a planet and moon transit was injected, on the right (80 light curves), only the planet. Each light curve consists of three consecutive transits. Each row of eight detrending methods uses the same light curve. The rows are sorted by their mean $\triangle \mathrm{BIC}$, with black lines indicating the $\triangle \mathrm{BIC}=\{-6,0,6\}$ positions for the mean $\triangle \mathrm{BIC}$ per row.

In Fig. 2.9, we show the $\triangle \mathrm{BIC}$ for the 160 simulated Kepler light curves that were not rejected by our detrending method due to gaps very close to a transit. The left panel shows 

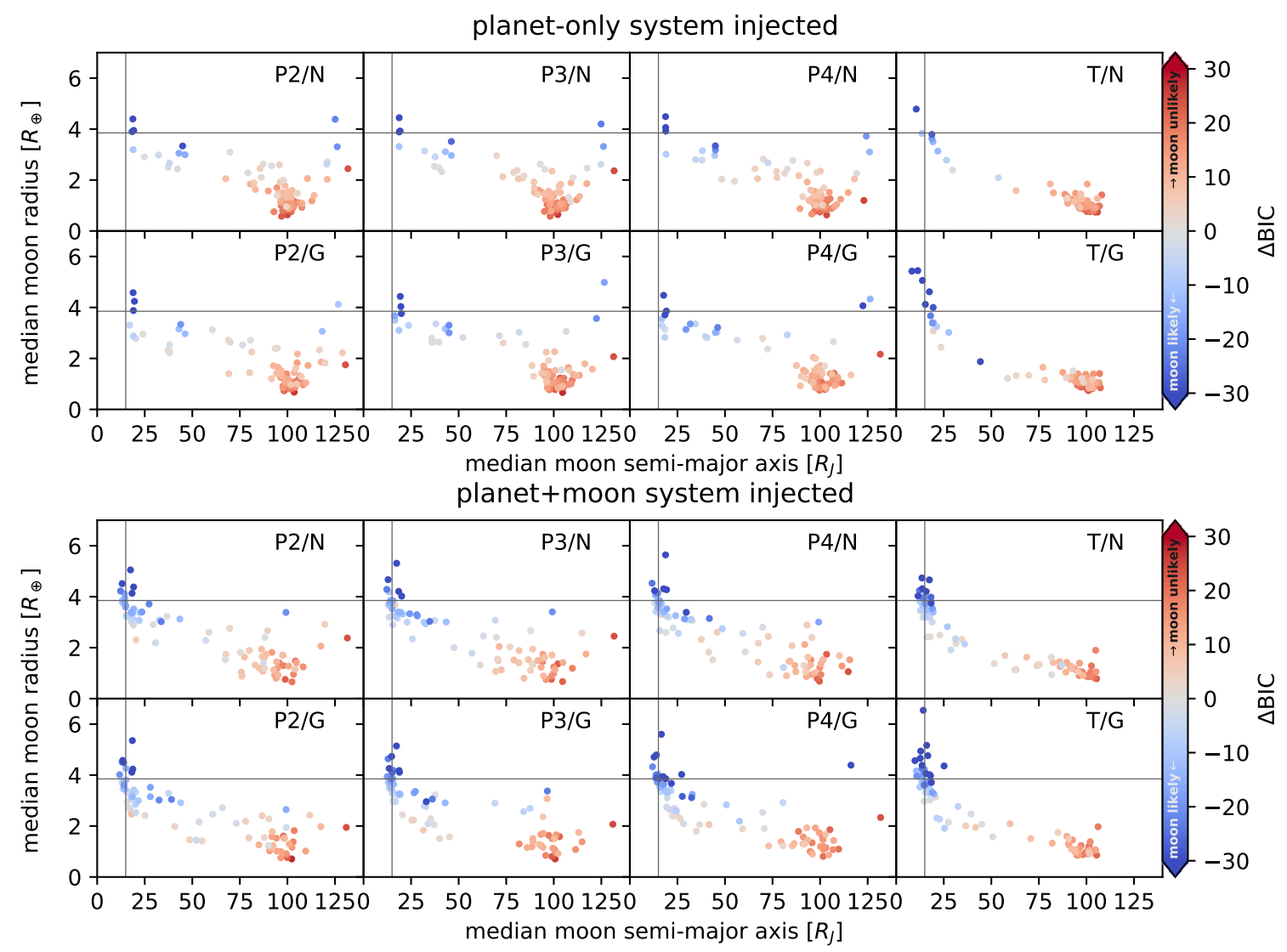

Figure 2.10: Distribution of the median likelihood $R_{\mathrm{s}}$ and $a_{\mathrm{s}}$ for the transits injected into different parts of the Kepler-1625 light curve, using different detrending methods. The $\triangle \mathrm{BIC}$ of the planet-only model compared to the planet-moon model is indicated by the symbol color. The values of the moon semimajor axis (abscissa) and radius (ordinate) suggested by Teachey et al. (2018) are indicated with thin, gray lines in each sub-panel.

our results for the analysis of planet-only injections and the right panel refers to planetmoon injections. The tables in the panel headers list the true negative, false positive, true positive, and false positive rates as well as the rates of ambiguous cases. With "positive" ("negative"), we here refer to the detection (non-detection) of a moon.

In particular, we find the true negative rate (left panel, $\triangle \mathrm{BIC} \geq 6$ ) to be between $65 \%$ and $87.5 \%$ and the true positive rate (right panel, $\triangle \mathrm{BIC} \leq-6$ ) to be between $31.25 \%$ and $46.25 \%$ depending on the detrending method, respectively.

The rates of false classifications is between $8.75 \%$ and $17.5 \%$ for the injected planetonly systems with a falsely detected moon (false positives) and between $30 \%$ and $41.25 \%$ for the injected planet-moon systems with a failed moon recovery (false negatives).

The rates of classification as a planet-moon system depend significantly on the treatment of gaps during the detrending procedure. Whenever the light curve is cut at a gap, the detection rates for a moon increase - both for the false positives and for the true positives. Among all the detrending methods, this effect is especially strong for the trigonometric detrending. The false positive rate increases by almost a factor of two from $8.75 \%(\mathrm{~T} / \mathrm{N})$ to $16.25 \%(\mathrm{~T} / \mathrm{G})$ and the true positive rate increases by $15 \%$ to $46.25 \%$. The effect on the true negative rate is strongest for the trigonometric detrending, decreasing from 
$87.5 \%$ when the light curve is not cut at gaps $(\mathrm{T} / \mathrm{N})$ to $72.5 \%$ if the light curve is cut $(\mathrm{T} / \mathrm{G})$. The false negative rate for the second-order polynomial detrending decreases from $41.25 \%(\mathrm{P} 2 / \mathrm{N})$ to $30 \%(\mathrm{P} 2 / \mathrm{G})$ when gaps are cut, while the false negative rates of the other detrending methods remain almost unaffected.

Of all the light curves with an injected planet only, $21.25 \%$ have an ambiguous classification with at least one of the detrending methods showing a negative $\triangle \mathrm{BIC}$ and a different method showing a positive $\triangle \mathrm{BIC}$ above the threshold. For the light curves with an injected planet-moon system, there are $18.75 \%$ with ambiguous classification and another $18.75 \%$ of the injected planet-moon systems are classified unanimously as true positives by all detrending methods.

Figure 2.10 shows the distribution of the retrieved moon parameters $a_{\mathrm{s}}$ and $R_{\mathrm{s}}$ as well as the corresponding $\triangle \mathrm{BIC}$ (see color scale) for each of the detrending methods.

For the light curves with an injected planet-moon system (lower set of panels), the maximum likelihood values of $a_{\mathrm{s}}$ and $R_{\mathrm{s}}$ of the true positives (blue) generally cluster around the injected parameters. In particular, we find that the moon turns out to be more likely (deeper-blue dots) when it is fitted to have a larger radius. The parameters of the false positives (blue dots in the upper set of panels) are more widely spread out, with moon radii ranging between 2 and $5 R_{\oplus}$ and the moon semimajor axes spread out through essentially the entire parameter range that we explored. The clustering of median $a_{\mathrm{s}}$ at around $100 R_{\mathrm{J}}$ is an artifact of taking the median over a very unlocalized distribution along $a_{\mathrm{s}}$. For the polynomial detrending methods, there are a certain number of what one could refer to as mischaracterized true positives. In these cases, the $\triangle \mathrm{BIC}$-based planet-only versus planet-moon classification is correct but the maximum likelihood values are very different from the injected ones.

The correctly identified planet-only systems show a similar distribution of $a_{\mathrm{s}}$ and $R_{\mathrm{s}}$ as in our experiment with white noise only and a 700 ppm amplitude (Fig. 2.5).

Most surprisingly, and potentially most worryingly, the false positives (blue dots in the upper set of panels in Fig. 2.10) cluster around the values of the moon parameters found by Teachey et al. (2018), in particular if the light curve is cut at the first gap.

\subsection{Discussion}

In this article, we compare several detrending methods of the light curve of Kepler-1625, some of which were used by Teachey et al. (2018) in their characterization of the exomoon candidate around Kepler-1625 b. However, we do not perform an exhaustive survey of all available detrending methods; for example we leave out Gaussian processes (Aigrain et al. 2016).

We show that the sequential detrending and fitting procedure of transit light curves is prone to introducing features that can be misinterpreted as signal, in our case as an exomoon. This "pre-whitening" method of the data must therefore be used with caution. Our investigations of a polynomial-based fitting and of a trigonometric detrending procedure show that the resulting best-fit model depends strongly on the specific detrending function; for example, on the order of the polynomial or on the minimum timescale (or wavelength) of a cosine. This is crucial for any search of secondary effects in the transit light curves - caused by moons, rings, evaporating atmospheres and so on - and is in 
stark contrast to a claim by Aizawa et al. (2017), who stated that neither the choice of the detrending function nor the choice of the detrending window of the light curve would have a significant effect on the result. We find that this might be true at the level of visual inspection by eye but not at the level of $100 \mathrm{ppm}$ or below. Part of the difference between our findings and those of Aizawa et al. (2017) could be in the different timescales we investigate. While they considered the effect of stellar flairs on timescales of less than a day, much less than the two-day transit duration of their specific target, our procedure operates on various timescales of up to several weeks. Moreover, we develop a dynamical moon model to fit multiple transits, whereas Aizawa et al. (2017) study only a single transit.

Since the actual presence and the putative orbital position of a hypothetical exomoon around Kepler-1625 b is unknown a priori, it is unclear how much of the light curve would need to be protected from (or neglected for) the pre-fit detrending process in order to avoid a detrending of a possible moon signal itself. In turn, we show that in the case of Kepler1625 , different choices for this protected timescale around the transit yield different confidences and different solutions for a planet-moon system. We find that the previously announced solution by Teachey et al. (2018) is only one of many possibilities with similar likelihoods (specifically: BIC). This suggests, but by no means proves, that all of these solutions could, in fact, be due to red noise artifacts (e.g. stellar or instrumental) rather than indicative of a moon signal.

Our finding of higher true positive rate compared to a false positive rate from injectionretrieval experiments could be interpreted as moderate evidence in favor of a genuine exomoon. This interpretation, however, depends on the number of transiting planets and planet candidates around stars with similar noise characteristics that were included in the Teachey et al. (2018) search. Broadly speaking, if more than a handful of similar targets are studied, the probability of at least one false positive detection becomes quite likely.

\subsection{Conclusions}

We investigated the detrending of the transit light curve of Kepler-1625 b with a method very similar to the one used by Teachey et al. (2018) and then applied a Bayesian framework with MCMC modeling to search for a moon. Our finding of a $\triangle \mathrm{BIC}$ of -4.954 favors the planet-moon over the planet-only hypothesis. Although significant, this tentative detection fails to cross the threshold of -6 , which we would consider strong evidence of a moon. Our $\triangle \mathrm{BIC}$ value would certainly change if we were to include the additional data from the high-precision transit observations executed in October 2017 with the Hubble Space Telescope (Teachey et al.2018) in our analysis. Moreover, by varying the free parameters of our detrending procedure, we also find completely different solutions for a planet-moon system, that is, different planet-moon orbital configurations during transits and different moon radii or planet-moon orbital semimajor axes.

As an extension to this validation of the previously published work, we performed 200 injection-retrieval experiments into the original out-of-transit parts of the Kepler light curve. We also extended the previous work by exploring different detrending methods, such as second-, third-, and fourth-order polynomials as well as trigonometric methods, and find false-positive rates between $8.75 \%$ and $16.25 \%$, depending on the method. Surprisingly, we find that the moon radius and planet-moon distances of these false positives 
are very similar to the ones measured by Teachey et al. (2018). In other words, in $8.75 \%$ to $16.25 \%$ of the light curves that contained an artificially injected planet only, we find a moon that is about as large as Neptune and orbits Kepler- $1625 \mathrm{~b}$ at about $20 R_{\mathrm{J}}$.

To summarize, we find tentative statistical evidence for a moon in this particular $\mathrm{Ke}-$ pler light curve of Kepler-1625, but we also show that a significant fraction of similar light curves, which contained a planet only, would nevertheless indicate a moon with properties similar to the candidate Kepler-1625 b-i. Clearly, stellar and systematic red noise components are the ultimate barrier to an unambiguous exomoon detection around Kepler-1625 b and follow-up observations have the potential of solving this riddle based on the framework that we present.

Of all the detrending methods we investigated, the trigonometric method, which is very similar to the CoFiAM method of Teachey et al.(2018), can produce the highest true positive rate. At the same time, however, this method also ranks among those producing the highest false positive rates as well. To conclude, we recommend that any future exomoon candidate be detrended with as many different detrending methods as possible to evaluate the robustness of the classification.

\subsection{Acknowledgements}

We thank James Kuszlewicz and Jesper Schou for useful discussions. This work was supported in part by the German Aerospace Center (DLR) under PLATO Data Center grant 50OL1701. This paper includes data collected by the Kepler mission. Funding for the Kepler mission is provided by the NASA Science Mission directorate. This work has made use of data provided by NASA and the Space Telescope Science Institute. K.R. is a member of the International Max Planck Research School for Solar System Science at the University of Göttingen. K.R. contributed to the analysis of the simulated light curves, to the interpretation of the results, and to the writing of the article. 


\section{Appendix}

\section{A Effect of the window length on the Bayesian infor- mation criterion}

Given the constraint of orbital stability, a moon can only possibly orbit its planet within the planet's Hill sphere. Therefore, transits may only occur within a certain time interval around the midpoint of the planetary transit. This time $t_{\text {Hill }}$ can be calculated as

$$
t_{\text {Hill }}=\frac{\eta R_{\text {Hill }}}{v_{\text {orbit }}}=\eta \frac{P}{2 \pi} \sqrt{\frac{M_{\mathrm{p}}}{3 M_{\star}}},
$$

where $v_{\text {orbit }}$ is the orbital velocity of the planet-moon system around the star, $M_{\mathrm{p}}$ and $M_{\star}$ the planet and star mass, $P$ the orbital period of the planet-moon system, and $R_{\text {Hill }}$ is the Hill radius of the planet. $\eta$ is a factor between 0 and 1 , which has been numerically determined for prograde moons $(\eta \approx 0.5)$ and for retrograde moons $(\eta \approx 1)$, details depending on the orbital eccentricities (Domingos et al. 2006). We focus on prograde
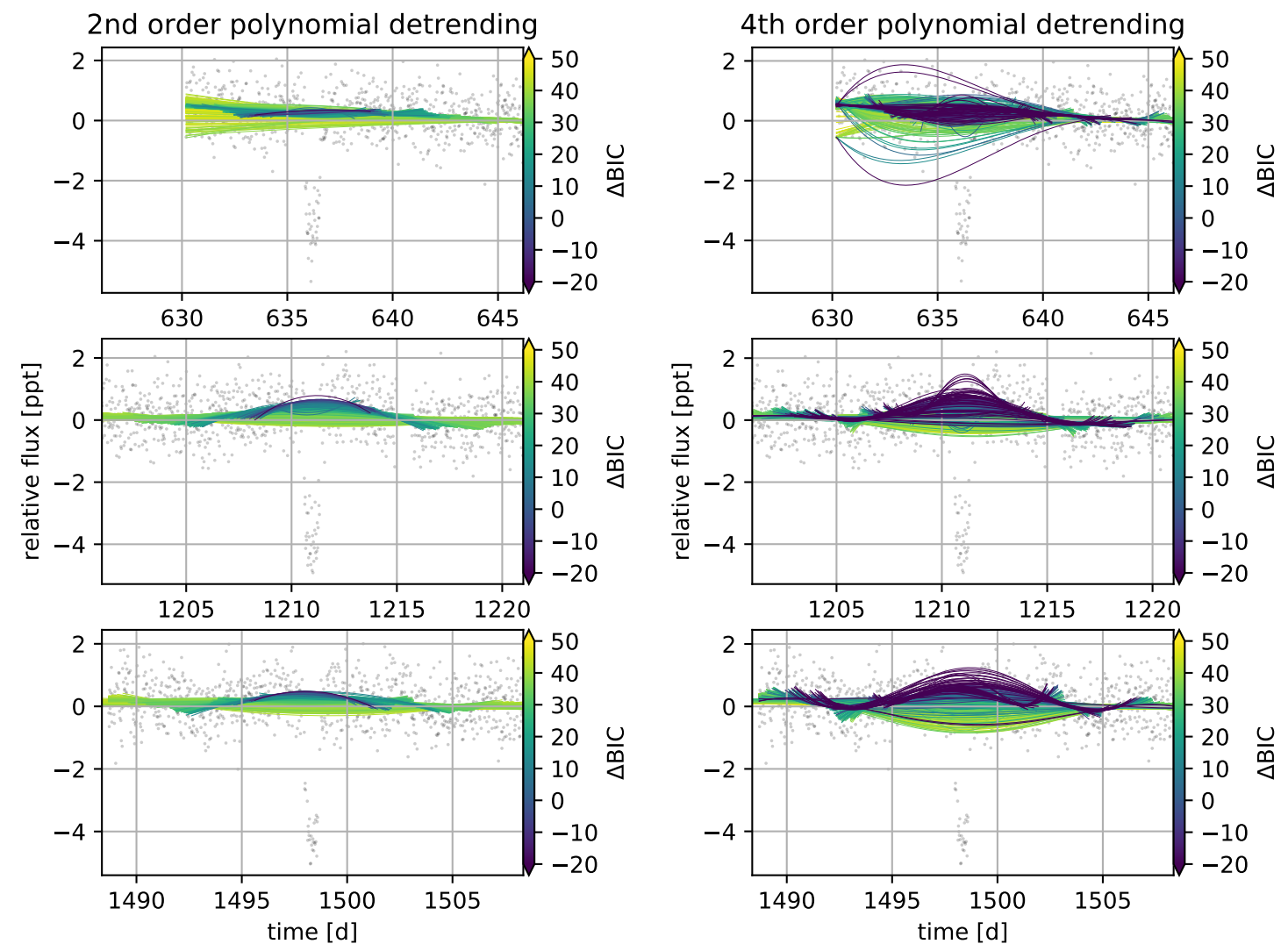

Figure 2.11: Detrending for different cutout times $t_{\mathrm{c}}$ and base length $D$, color coded by the resulting $\triangle \mathrm{BIC}$ using a second- and fourth-order polynomial function. While some of the detrending models corresponding to a large negative $\triangle \mathrm{BIC}$ are clearly the result of incorrect detrending, it is much less clear for many other detrending models. 
moons and choose $\eta=0.5$. For a $10 M_{\mathrm{J}}$ planet in a $287 \mathrm{~d}$ orbit around a $1.1 M_{\odot}$ star the Hill time is $t_{\text {Hill }}=3.25 \mathrm{~d}$.

As shown in Fig. 2.2, the length of the light curve, which is neglected for the polynomial fit has a strong effect on the resulting detrended light curve. Figure 2.11 shows the effect that different cutout times $t_{c}$ and detrending base lines $D$ can have on whether a moon is detected or not.

\section{B Autocorrelation of detrended light curves}

The autocorrelations of the detrended light curves are shown in Fig. 2.12. For all three transits, the autocorrelation is close to zero, except for the zero-lag component. This suggests that it is reasonable to model the noise covariance matrix as a diagonal matrix (see Sect. 2.2.2.3).

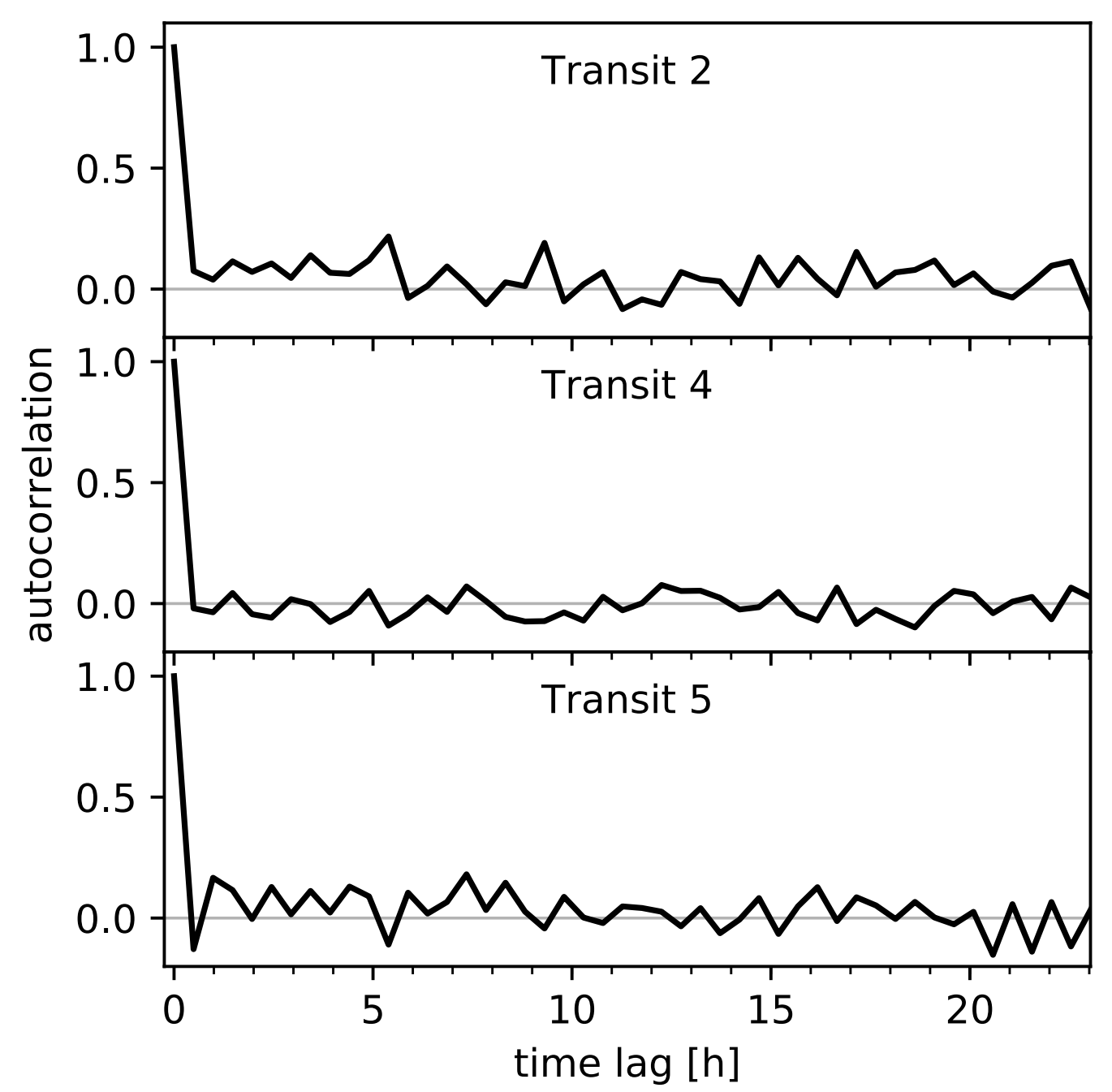

Figure 2.12: The autocorrelation of the difference between the detrended light curve and the best fitting model. 


\title{
3 Paper II: An alternative interpretation of the exomoon candidate signal in the combined Kepler and Hubble data of Kepler-1625*
}

\author{
René Heller ${ }^{1}$, Kai Rodenbeck ${ }^{2,1}$, and Giovanni Bruno ${ }^{3}$ \\ ${ }^{1}$ Max Planck Institute for Solar System Research, Justus-von-Liebig-Weg 3, 37077 Göttingen, \\ Germany \\ ${ }^{2}$ Institute for Astrophysics, Georg August University Göttingen, Friedrich-Hund-Platz 1, 37077 \\ Göttingen, Germany \\ ${ }^{3}$ INAF, Astrophysical Observatory of Catania, Via S. Sofia 78, 95123 Catania, Italy
}

\begin{abstract}
Context: Kepler and Hubble photometry of a total of four transits by the Jupiter-sized exoplanet Kepler-1625 b have recently been interpreted to show evidence of a Neptunesized exomoon. The key arguments were an apparent drop in stellar brightness after the planet's October 2017 transit seen with Hubble and its 77.8 min early arrival compared to a strictly periodic orbit.

Aims: The profound implications of this first possible exomoon detection and the physical oddity of the proposed moon, i.e., its giant radius prompt us to examine the planet-only hypothesis for the data and to investigate the reliability of the Bayesian information criterion (BIC) used for detection.

Methods: We combined Kepler's Pre-search Data Conditioning Simple Aperture Photometry (PDCSAP) with the previously published Hubble light curve. In an alternative approach, we performed a synchronous polynomial detrending and fitting of the Kepler

*This chapter reproduces the article An alternative interpretation of the exomoon candidate signal in the combined Kepler and Hubble data of Kepler-1625 by R. Heller, K. Rodenbeck, G. Bruno, published in Astronomy and Astrophysics 624, A95 (2019), reproduced with permission (C) ESO. Contributions: RH wrote the manuscript, proposed Figs. 3.2-3.4 generated Fig. 3.5, and guided the work. KR derived the starplanet-moon orbital simulations and the respective statistics and generated Figs. 3.2. 3.4 . GB performed the light curve extraction from the WFC3 Hubble data and generated Fig. 3.1. All authors contributed equally to the interpretation of the data.
\end{abstract}


3 Paper II: An alternative interpretation of the exomoon candidate signal in the combined Kepler and Hubble data of Kepler-1625

data combined with our own extraction of the Hubble photometry. We generated five million parallel-tempering Markov chain Monte Carlo (PTMCMC) realizations of the data with both a planet-only model and a planet-moon model, and compute the BIC difference $(\triangle \mathrm{BIC})$ between the most likely models, respectively.

Results: The $\triangle \mathrm{BIC}$ values of -44.5 (using previously published Hubble data) and -31.0 (using our own detrending) yield strong statistical evidence in favor of an exomoon. Most of our orbital realizations, however, are very different from the best-fit solutions, suggesting that the likelihood function that best describes the data is non-Gaussian. We measure a 73.7 min early arrival of Kepler-1625 b for its Hubble transit at the $3 \sigma$ level. This deviation could be caused by a $1 \mathrm{~d}$ data gap near the first Kepler transit, stellar activity, or unknown systematics, all of which affect the detrending. The radial velocity amplitude of a possible unseen hot Jupiter causing the Kepler-1625 b transit timing variation could be approximately $100 \mathrm{~m} \mathrm{~s}^{-1}$.

Conclusions: Although we find a similar solution to the planet-moon model to that previously proposed, careful consideration of its statistical evidence leads us to believe that this is not a secure exomoon detection. Unknown systematic errors in the Kepler/Hubble data make the $\triangle \mathrm{BIC}$ an unreliable metric for an exomoon search around Kepler-1625 b, allowing for alternative interpretations of the signal.

\subsection{Introduction}

The recent discovery of an exomoon candidate around the transiting Jupiter-sized object Kepler-1625 b orbiting a slightly evolved solar mass star (Teachey et al. 2018) came as a surprise to the exoplanet community. This Neptune-sized exomoon, if confirmed, would be unlike any moon in the solar system, it would have an estimated mass that exceeds the total mass of all moons and rocky planets of the solar system combined. It is currently unclear how such a giant moon could have formed (Heller 2018a).

Rodenbeck et al. (2018) revisited the three transits obtained with the Kepler space telescope between 2009 and 2013 and found marginal statistical evidence for the proposed exomoon. Their transit injection-retrieval tests into the out-of-transit Kepler data of the host star also suggested that the exomoon could well be a false positive. A solution to the exomoon question was supposed to arrive with the new Hubble data of an October 2017 transit of Kepler-1625 b (Teachey and Kipping 2018).

The new evidence for the large exomoon by Teachey and Kipping (2018), however, remains controversial. On the one hand, the Hubble transit light curve indeed shows a significant decrease in stellar brightness that can be attributed to the previously suggested moon. Perhaps more importantly, the transit of Kepler-1625 b occurred 77.8 min earlier than expected from a sequence of strictly periodic transits, which is in very good agreement with the proposed transit of the exomoon candidate, which occurred before the planetary transit. On the other hand, an upgrade of Kepler's Science Operations Center pipeline from version 9.0 to version 9.3 caused the exomoon signal that was presented in the Simple Aperture Photometry (SAP) measurements in the discovery paper (Teachey et al. 2018) to essentially vanish in the SAP flux used in the new study of Teachey and Kipping (2018). This inconsistency, combined with the findings of Rodenbeck et al. (2018) that demonstrate that the characterization and statistical evidence for this exo- 
moon candidate depend strongly on the methods used for data detrending, led us to revisit the exomoon interpretation in light of the new Hubble data.

Here we address two questions. How unique is the proposed orbital solution of the planet-moon system derived with the Bayesian information criterion (BIC)? What could be the reason for the observed 77.8 min difference in the planetary transit timing other than an exomoon?

\subsection{Methods}

Our first goal was to fit the combined Kepler and Hubble data with our planet-moon transit model (Rodenbeck et al. 2018) and to derive the statistical likelihood for the data to represent the model. In brief, we first model the orbital dynamics of the star-planet-moon system using a nested approach, in which the planet-moon orbit is Keplerian and unperturbed by the stellar gravity. The transit model consists of two black circles, one for the planet and one for the moon, that pass in front of the limb-darkened stellar disk. The resulting variations in the stellar brightness are computed using Ian Crossfield's python code of the Mandel and Agol (2002) analytic transit model 11 The entire model contains 16 free parameters and it features three major updates compared to Rodenbeck et al. (2018): (1) Planet-moon occultations are now correctly simulated, (2) the planet's motion around the local planet-moon barycenter is taken into account, and (3) inclinations between the circumstellar orbit of the planet-moon barycenter and the planet-moon orbit are now included.

We used the emcee code ${ }^{2}$ of Foreman-Mackey et al. (2013) to generate Markov chain Monte Carlo (MCMC) realizations of our planet-only model $\left(\mathcal{M}_{0}\right)$ and planet-moon model $\left(\mathcal{M}_{1}\right)$ and to derive posterior probability distributions of the set of model parameters $(\vec{\theta})$. We tested both a standard MCMC sampling with 100 walkers and a parallel-tempering ensemble MCMC (PTMCMC) with five temperatures, each of which has 100 walkers. As we find a better convergence rate for the PTMCMC sampling, we use it in the following. Moreover, PTMCMC can sample both the parameter space at large and in regions with tight peaks of the likelihood function. The PTMCMC sampling is allowed to walk five million steps.

The resulting model light curves are referred to as $\mathcal{F}_{i}(t, \vec{\theta})$, where $t$ are the time stamps of the data points from Kepler and Hubble ( $N$ measurements in total), for which timeuncorrelated standard deviations $\sigma_{j}$ at times $t_{j}$ are assumed, following the suggestion of Teachey and Kipping (2018). This simplifies the joint probability density of the observed (and detrended) flux measurements $(F(t))$ to the product of the individual probabilities for each data point,

$$
p\left(F \mid \vec{\theta}, \mathcal{M}_{i}\right)=\prod_{j=1}^{N} \frac{1}{\sqrt{2 \pi \sigma_{j}^{2}}} \exp \left(-\frac{\left(F\left(t_{j}\right)-\mathcal{F}_{i}\left(t_{j}, \vec{\theta}\right)\right)^{2}}{2 \sigma_{j}^{2}}\right)
$$

\footnotetext{
${ }^{1}$ Available at www.astro.ucla.edu/ ianc/files

${ }^{2}$ Available at http://dfm.io/emcee/current/user/pt
} 
3 Paper II: An alternative interpretation of the exomoon candidate signal in the combined Kepler and Hubble data of Kepler-1625

We then determined the set of parameters ( $\left.\vec{\theta}_{\max }\right)$ that maximizes the joint probability density function $\left(p\left(F \mid \vec{\theta}_{\max }, \mathcal{M}_{i}\right)\right)$ for a given light curve $F\left(t_{j}\right)$ and model $\mathcal{M}_{i}$ and calculated the BIC (Schwarz 1978)

$$
\operatorname{BIC}\left(\mathcal{M}_{i} \mid F\right)=m_{i} \ln N-2 \ln p\left(F \mid \vec{\theta}_{\max }, \mathcal{M}_{i}\right) .
$$

The advantage of the BIC in comparison to $\chi^{2}$ minimization, for example, is in its relation to the number of model parameters $\left(m_{i}\right)$ and data points. The more free parameters in the model, the stronger the weight of the first penalty term in Eq. (3.2), thereby mitigating the effects of overfitting. Details of the actual computer code implementation or transit simulations aside, this Bayesian framework is essentially what the Hunt for Exomoons with Kepler survey used to identify and rank exomoon candidates (Kipping et al. 2012), which ultimately led to the detection of the exomoon candidate around Kepler-1625 b after its first detection via the orbital sampling effect (Heller 2014, Heller et al. 2016a).

\subsubsection{Data preparation}

In a first step, we used Kepler's Pre-search Data Conditioning Simple Aperture Photometry (PDCSAP) and the Hubble Wide Field Camera 3 (WFC3) light curve as published by Teachey and Kipping (2018) based on their quadratic detrending. Then we executed our PTMCMC fitting and derived the $\triangle \mathrm{BIC}$ values and the posterior parameter distributions.

\subsubsection{Extraction of the Hubble light curve}

In a second step, we did our own extraction of the Hubble light curve including an exponential ramp correction for each Hubble orbit. Then we performed the systematic trend correction together with the transit fit of a planet-moon model. Our own detrending of the light curves is not a separate step, but it is integral to the fitting procedure. For each calculation of the likelihood, we find the best fitting detrending curve by dividing the observed light curve by the transit model and by fitting a third-order polynomial to the resulting light curve. Then we remove the trend from the original light curve by dividing it through the best-fit detrending polynomial and evaluate the likelihood. We also performed a test in which the detrending parameters were free PTMCMC model parameters and found similar results for the parameter distributions but at a much higher computational cost. We note that the resulting maximum likelihood is (and must be) the same by definition if the PTMCMC sampling converges.

Kepler-1625 was observed by Hubble under the GO program 15149 (PI Teachey). The observations were secured from October 28 to 29, 2017, to cover the $\sim 20 \mathrm{hr}$ transit plus several hours of out-of-transit stellar flux (Teachey and Kipping 2018). The F130N filter of WFC3 was used to obtain a single direct image of the target, while 232 spectra were acquired with the G141 grism spanning a wavelength range from 1.1 to $1.7 \mu \mathrm{m}$. Due to the faintness of the target, it was observed in staring mode (e.g. Berta et al.2012, Wilkins et al. 2014) unlike the most recent observations of brighter exoplanet host stars, which were monitored in spatial scanning mode (McCullough and MacKenty 2012). Hence, instead of using the IMA files as an intermediate product, we analyzed the FLT files, which are the final output of the calwfc3 pipeline of Hubble and allow a finer manipulation of 
the exposures during consecutive nondestructive reads. Each FLT file contains measurements between about 100 and 300 electrons per second, with exposure times of about 291 seconds.

We used the centroid of the stellar image to calculate the wavelength calibration, adopting the relations of Pirzkal et al. (2016). For each spectroscopic frame, we first rejected the pixels flagged by calwfc3 as "bad detector pixels", pixels with unstable response, and those with uncertain flux value (Data Quality condition 4, 32, or 512). Then we corrected each frame with the flat field file available on the Space Telescope Science Institute (STScI) website ${ }^{3}$ by following the prescription of the WFC3 online manual. We performed the background subtraction on a column-by-column basis. Due to a number of contaminant stars in the observation field (Fig. 3.1. top panel), we carefully selected a region on the detector that was as close as possible to the spectrum of Kepler-1625, close to row 150 in spatial dimension, and far from any contaminant. For each column on the detector, we applied a $5 \sigma$ clipping to reject the outliers and then calculated the median background flux value in that column. Following STScI prescriptions, we also removed pixels with an electron-per-second count larger than 5. An example for the background behavior is shown in the bottom panel of Fig. 3.1.

We inspected each frame with the image registration package (Baker and Matthews 2001) to search drifts in both axes of the detector with respect to the very last frame, and then extracted the spectrum of Kepler-1625 by performing optimal extraction (Horne 1986) on the detector rows containing the stellar flux. This procedure automatically removes bad pixels and cosmic rays from the frames by correcting them with a smoothing function. We started the extraction with an aperture of a few pixels centered on the peak of the stellar trace and gradually increased its extension by one pixel per side on the spatial direction until the flux dispersion reached a minimum.

We performed another outlier rejection by stacking all the one-dimensional spectra along the time axis. We computed a median-filtered version of the stellar flux at each wavelength bin and performed a $3 \sigma$ clipping between the computed flux and the median filter. Finally, we summed the stellar flux across all wavelength bins from 1.115 to 1.645 $\mu \mathrm{m}$ to obtain the band-integrated stellar flux corresponding to each exposure.

Before performing the PTMCMC optimization, we removed the first Hubble orbit from the data set and the first data point of each Hubble orbit, as they are affected by stronger instrumental effects than the other observations (Deming et al. 2013) and cannot be corrected with the same systematics model. We also removed the last point of the 12th, 13th, and 14th Hubble orbit since they were affected by the passage of the South Atlantic Anomaly (as highlighted in the proposal file, available on the STScI website).

\subsubsection{Proposed unseen planet}

\subsubsection{Mass-orbit constraints for a close-in planet}

According to Teachey et al. (2018), the 2017 Hubble transit of Kepler-1625 b occurred about 77.8 min earlier than predicted, an effect that could be astrophysical in nature and is referred to as a transit timing variation (TTV). As proposed by Teachey et al. (2018),

$\sqrt[3]{\text { www.stsci.edu/hst/wfc3/analysis/grism_obs/calibrations/ }}$ wfc3_g141.html 
3 Paper II: An alternative interpretation of the exomoon candidate signal in the combined Kepler and Hubble data of Kepler-1625
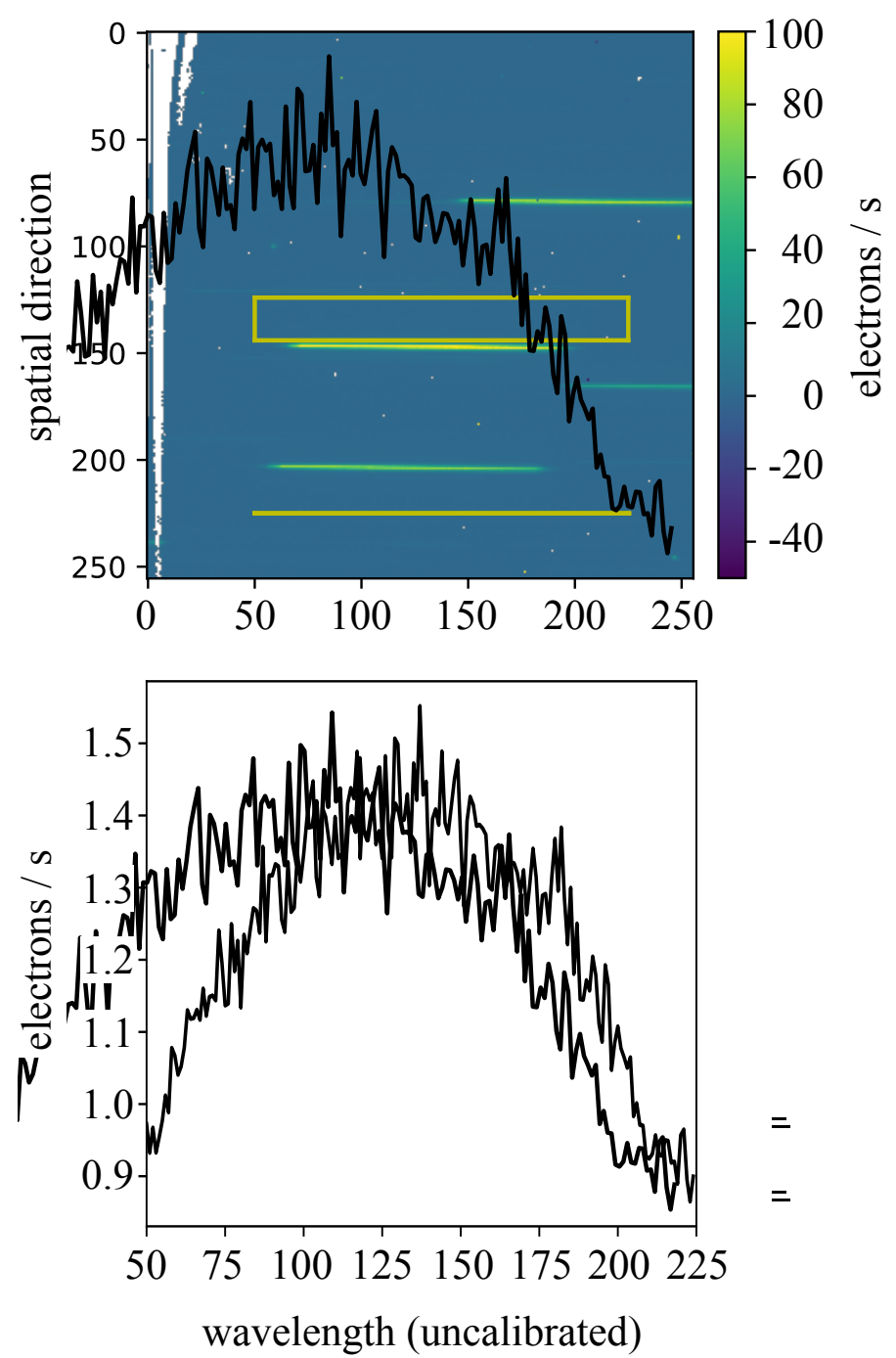

Figure 3.1: Top: Example of a WFC3 exposure of Kepler-1625. The abscissa shows the column pixel prior to wavelength calibration. The yellow box indicates the region used for background estimation. The spectrum of Kepler-1625 is at the center of the frame, around row 150 in the spatial direction, while several contaminant sources are evident in other regions of the detector. The color bar illustrates the measured charge values. Bottom: Background value measured across the rows of the same frame.

this TTV could either be interpreted as evidence for an exomoon or it could indicate the presence of a hitherto unseen additional planet. Various planetary configurations can cause the observed TTV effect such as an inner planet or an outer planet. At this point, no stellar radial velocity measurements of Kepler-1625 exist that could be used to search for additional nontransiting planets in this system.

In the following, we focus on the possibility of an inner planet with a much smaller orbital period than Kepler-1625 b simply because it would have interesting observational consequences. We use the approximation of Agol et al. (2005) for the TTV amplitude $(\delta t)$ due to a close inner planet, which would impose a periodic variation on the position of 

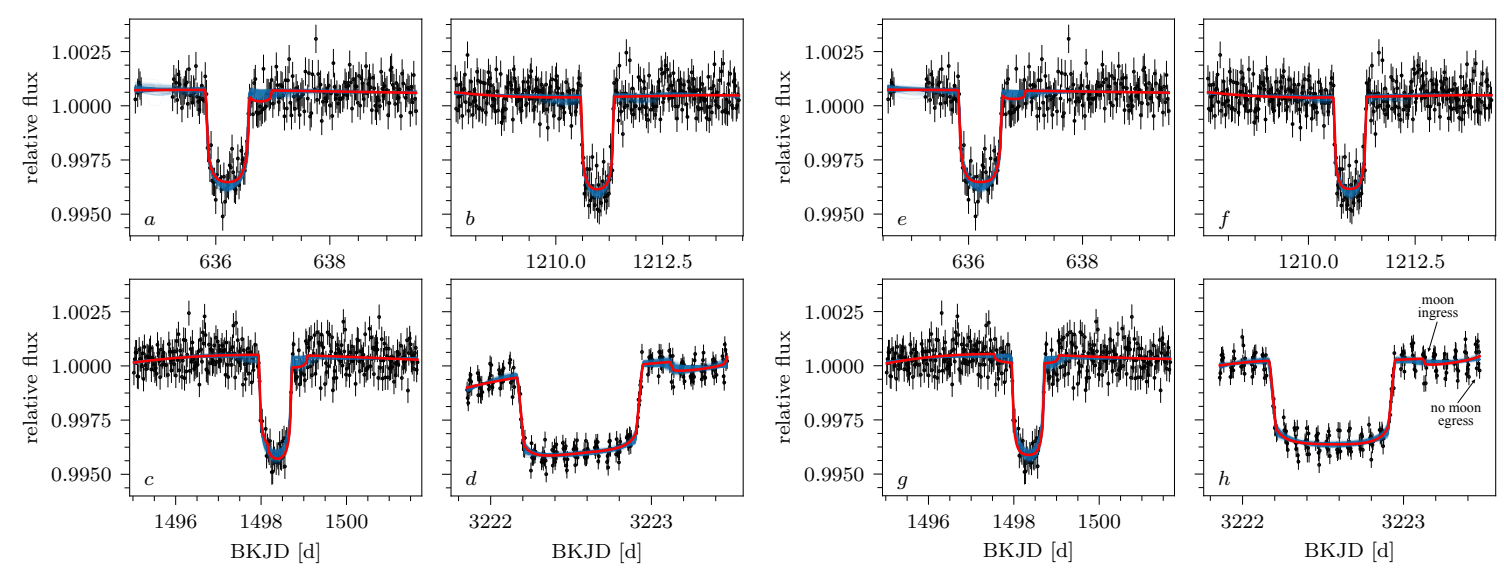

Figure 3.2: Orbital solutions for Kepler-1625 b and its suspected exomoon based on the combined Kepler and Hubble data. (a,b,c) Kepler PDCSAP flux and $(d)$ the quadratic detrending of the Hubble data from Teachey and Kipping (2018). The blue curves show 1000 realizations of our PTMCMC fitting of a planet-moon model. Our most likely solution (red line) is very similar to the one found by Teachey and Kipping (2018), but differs significantly from the one initially found by Teachey et al. (2018). $(e, f, g)$ Kepler PDCSAP flux and $(h)$ our own detrending of the Hubble light curve (in parallel to the fitting). The ingress and egress of the model moon are denoted with arrows and labels in panel $h$ as an example.

the star, and solve their expression for the mass of the inner planet $\left(M_{\mathrm{p}, \text { in }}\right)$ as a function of its orbital semimajor axis $\left(a_{\mathrm{p}, \text { in }}\right)$,

$$
M_{\mathrm{p}, \text { in }}=\delta t M_{\star} \frac{a_{\mathrm{p}, \text { out }}}{a_{\mathrm{p}, \text { in }} P_{\mathrm{p}, \text { out }}},
$$

where $a_{\text {out }}=0.87 \mathrm{AU}$ is the semimajor axis of Kepler-1625 b. The validity of this expression is restricted to coplanar systems without significant planet-planet interaction and with $a_{\text {out }} \gg a_{\text {in }}$, so that TTVs are only caused by the reflex motion of the star around its barycenter with the inner planet.

As we show in Sect. 3.3.2, the proposed inner planet could be a hot Jupiter. The transits of a Jupiter-sized planet, however, would be visible in the Kepler data. As a consequence, we can estimate the minimum orbital inclination ( $i$ ) between Kepler-1625 b and the suspected planet to prevent the latter from showing transits. This angle is given as per $i=\arctan \left(R_{\star} / a_{\mathrm{p}, \text { in }}\right)$ and we use $R_{\star}=1.793_{-0.488}^{+0.263} R_{\odot}$ (Mathur et al. 2017).

\subsubsection{Orbital stability}

We can exclude certain masses and orbital semimajor axes for an unseen inner planet based on the criterion of mutual Hill stability. This instability region depends to some extent on the unknown mass of Kepler-1625 b. Mass estimates can be derived from a star-planet-moon model, but these estimates are irrelevant if the observed TTVs are due to an unseen planetary perturber. Hence, we assume a nominal Jupiter mass $\left(M_{\mathrm{Jup}}\right)$ for Kepler-1625 b. 
3 Paper II: An alternative interpretation of the exomoon candidate signal in the combined Kepler and Hubble data of Kepler-1625
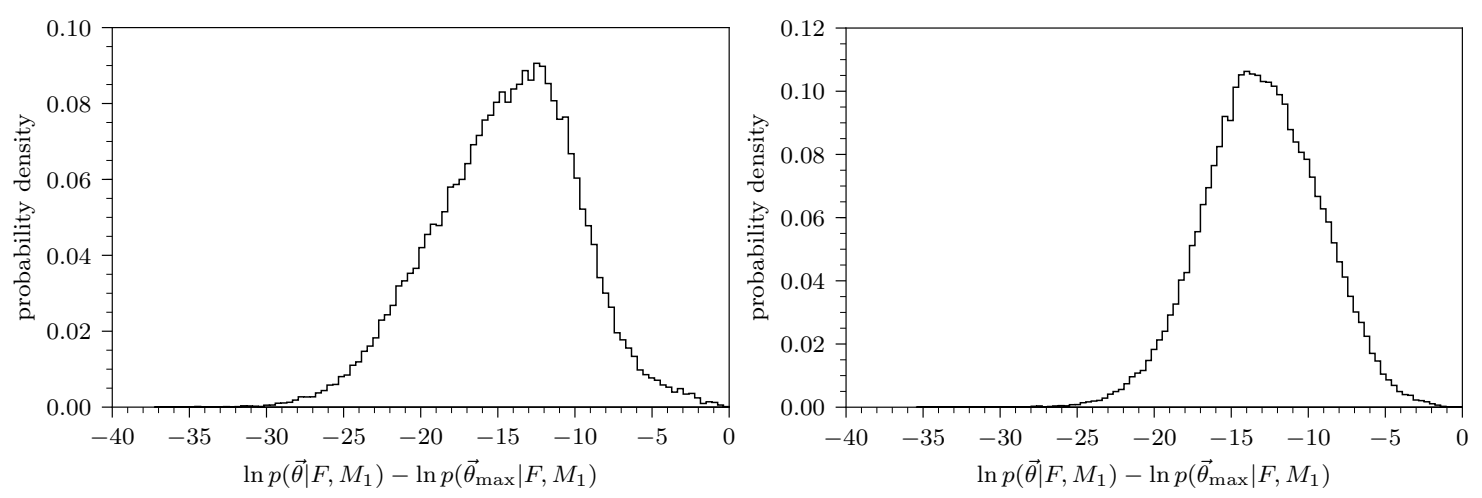

Figure 3.3: Differential likelihood distribution between the most likely planet-moon model and the other solutions using $10^{6}$ steps of our PTMCMC fitting procedure. Left: Results from fitting our planet-moon transit model to the original data from Teachey and Kipping (2018). Right: Results from fitting our planet-moon transit model to our own detrending of the Kepler and WFC3 data. In both panels the most likely model is located at 0 along the abscissa by definition. In both cases the models do not converge to the best-fit solution, suggesting that the best-fit solution could in fact be an outlier.

The Hill sphere of a planet with an orbital semimajor axis $a_{\mathrm{p}}$ around a star with mass $M_{\star}$ can be estimated as $R_{\mathrm{H}}=a_{\mathrm{p}}\left(M_{\mathrm{p}} /\left[3 M_{\star}\right]\right)^{1 / 3}$, which suggests $R_{\mathrm{H}}=125 R_{\mathrm{Jup}}$ for Kepler-1625 b. We calculate the Hill radius of the proposed inner planet accordingly, and identify the region in the mass-semimajor axis diagram of the inner planet that would lead to an overlap of the Hill spheres and therefore to orbital instability.

\subsection{Results}

\subsubsection{PTMCMC sampling and $\triangle \mathrm{BIC}$}

Regarding the combined data set of the Kepler and Hubble data as detrended by Teachey and Kipping (2018), we find a $\triangle \mathrm{BIC}$ of -44.5 between the most likely planet-only and the most likely planet-moon solution. A combination of the Kepler and Hubble light curves based on our own extraction of the WFC3 data yields a $\triangle \mathrm{BIC}$ of -31.0. Formally speaking, both of these two values can be interpreted as strong statistical evidence for an exomoon interpretation. The two values are very different, however, which suggests that the detrending of the Hubble data has a significant effect on the exomoon interpretation. In other words, this illustrates that the systematics are not well-modeled and poorly understood.

In Fig. $3.2 \mathrm{a}-\mathrm{d}$, we show our results for the PTMCMC fitting of our planet-moon model to the four transits of Kepler-1625 b including the Hubble data as extracted and detrended by Teachey and Kipping (2018) using a quadratic fit. Although our most likely solution shows some resemblance to the one proposed by Teachey and Kipping (2018), we find that several aspects are different. As an example, the second Kepler transit (Fig. 3.2b) is fitted best without a significant photometric moon signature, that is to say, the moon 

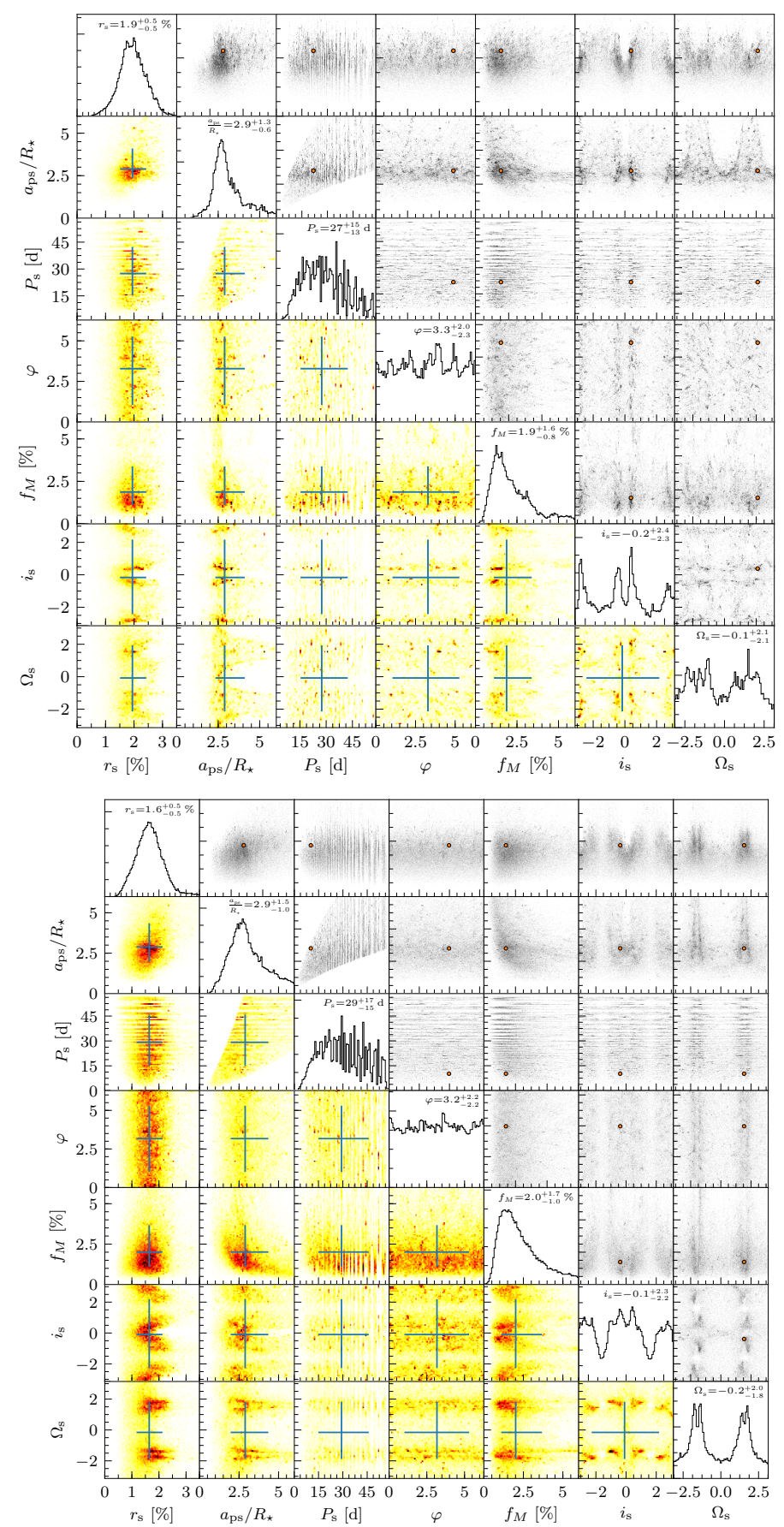

Figure 3.4: Posterior distributions of a parallel tempering ensemble MCMC sampling of the combined Kepler and WFC3 data with our planet-moon model. Top: Results for the original data from Teachey and Kipping (2018). Bottom: Results for our own detrending of the Kepler and WFC3 data. In both figures, scatter plots are shown with black dots above the diagonal, and projected histograms are shown as colored pixels below the diagonal. The most likely parameters are denoted with an orange point in the scatter plots. Histograms of the moon-to-star radius ratio $r_{\mathrm{s}}$, scaled semimajor axis of the planet-moon system $\left(a_{\mathrm{ps}} / R_{\star}\right)$, satellite orbital period $\left(P_{\mathrm{s}}\right)$, satellite orbital phase $(\varphi)$, moon-to-planet mass ratio $\left(f_{\mathrm{M}}\right)$, orbital inclination of the satellite with respect to our line of sight $\left(i_{\mathrm{s}}\right)$, and the orientation of the ascending node of the satellite orbit $\left(\Omega_{\mathrm{s}}\right)$ are shown on the diagonal. Median values and standard deviations are indicated with error bars in the histograms. 
3 Paper II: An alternative interpretation of the exomoon candidate signal in the combined Kepler and Hubble data of Kepler-1625

does not pass in front of the stellar disk 4 , whereas the corresponding best-fit model of Teachey and Kipping (2018) shows a clear dip prior to the planetary transit (see their Fig. 4). What is more, most of our orbital solutions (blue lines) differ substantially from the most likely solution (red line). In other words, the orbital solutions do not converge and various planet-moon orbital configuration are compatible with the data, though with lower likelihood.

In Fig. 3.2 $\mathrm{e}-\mathrm{h}$, we illustrate our results for the PTMCMC fitting of our planet-moon model to the four transits of Kepler-1625 b including our own extraction and detrending of the Hubble transit. Again, the orbital solutions (blue lines) do not converge. A comparison of panels $\mathrm{d}$ and $\mathrm{h}$ shows that the different extraction and detrending methods do have a significant effect on the individual flux measurements, in line with the findings of Rodenbeck et al. (2018). Although the time of the proposed exomoon transit is roughly the same in both panels, we find that the best-fit solution for the data detrended with our own reduction procedure does not contain the moon egress (panel $\mathrm{h}$ ), whereas the best-fit solution of the data detrended by Teachey and Kipping (2018) does contain the moon egress (panel d). A similar fragility of this particular moon egress has been noted by Teachey and Kipping (2018) as they explored different detrending functions (see their Fig. 3).

Our Fig. 3.3 illustrates the distribution of the differential likelihood for the planetmoon model between the most likely model parameter set $\left(\vec{\theta}_{\max }\right)$ and the parameter sets $\left(\vec{\theta}^{\prime}\right)$ found after five million steps of our PTMCMC fitting procedure, $p\left(\vec{\theta}^{\prime} \mid F, \mathcal{M}_{1}\right)-$ $p\left(\vec{\theta}_{\max } \mid F, \mathcal{M}_{1}\right)$. For the combined Kepler and Hubble data detrended by Teachey and Kipping (2018) (left panel) and for our own Hubble data extraction and detrending (right panel), we find that most model solutions cluster around a differential likelihood that is very different from the most likely solution, suggesting that the most likely model is, in some sense, a statistical outlier. We initially detected this feature after approximately the first one hundred thousand PTMCMC fits. Hence, we increased the number of PTMCMC samplings to half a million and finally to five million to make sure that we sample any potentially narrow peaks of the likelihood function near the best-fit model at $p\left(\vec{\theta}^{\prime} \mid F, \mathcal{M}_{1}\right)-p\left(\vec{\theta}_{\text {max }} \mid F, \mathcal{M}_{1}\right)=0$ with sufficient accuracy. We find, however, that this behavior of the differential likelihood distribution clustering far from the best-fit solution persists, irrespective of the available computing power devoted to the sampling.

Figure 3.4 shows the posterior distributions of the moon parameters of our planetmoon model. The top panel refers to our PTMCMC fitting of the combined Kepler and Hubble data (Hubble data as detrended and published by Teachey and Kipping|2018), and the bottom panel shows our PTMCMC fitting of the Kepler data combined with our own extraction and detrending of the Hubble light curve. The respective median values and standard deviations are noted in the upper right corners of each subpanel and summarized in Table 3.1

A comparison between the upper and lower corner plots in Fig. 3.4 reveals that the different detrending and fitting techniques have a significant effect on the resulting posterior distributions, in particular for $i_{\mathrm{s}}$ and $\Omega_{\mathrm{s}}$, the two angles that parameterize the orientation of the moon orbit. At the same time, however, the most likely values (red dots above the plot diagonal) and median values (blue crosses below the plot diagonal) of the seven parameters shown are well within the $1 \sigma$ tolerance.

\footnotetext{
${ }^{4}$ Martin et al. (2019) estimate that failed exomoon transits should actually be quite common for misaligned planet-moon systems, such as the one proposed by Teachey and Kipping (2018).
} 
Table 3.1: Results of our PTMCMC fitting procedure to the combined Kepler and Hubble data. The Hubble data was either based on the photometry extracted by Teachey and Kipping (2018, TK18b, central column) or based on our own extraction (right column).

\begin{tabular}{ccc}
\hline \hline & TK18b $H S T$ photometry & our $H S T$ photometry \\
\hline$r_{\mathrm{S}}[\%]$ & $1.9_{-0.5}^{+0.5}$ & $1.6_{-0.5}^{+0.5}$ \\
$a_{\mathrm{ps}}\left[R_{\star}\right]$ & $2.9_{-0.6}^{+1.3}$ & $2.9_{-1.0}^{+1.5}$ \\
$P_{\mathrm{S}}[\mathrm{d}]$ & $27_{-13}^{+15}$ & $29_{-15}^{+17}$ \\
$\varphi[\mathrm{rad}]$ & $3.3_{-2.3}^{+2.0}$ & $3.2_{-2.2}^{+2.2}$ \\
$f_{\mathrm{M}}[\%]$ & $1.9_{-0.8}^{+1.6}$ & $2.0_{-1.0}^{+1.7}$ \\
$i_{\mathrm{S}}[\mathrm{rad}]$ & $-0.2_{-2.3}^{+2.4}$ & $-0.1_{-2.2}^{+2.3}$ \\
$\Omega_{\mathrm{s}}[\mathrm{rad}]$ & $-0.1_{-2.1}^{+2.1}$ & $-0.2_{-1.8}^{+2.0}$ \\
\hline
\end{tabular}

Notes. Figure 3.4 illustrates quite clearly that the posterior distributions are not normally distributed and often not even representative of skewed normal distributions. The confidence intervals stated in this table have thus to be taken with care.

The following features can be observed in both panels of Fig. 3.4. The moon-to-star radius ratio (Col. 1, leftmost) shows an approximately normal distribution, whereas the scaled planet-moon orbital semimajor axis (Col. 2) shows a more complicated, skewed distribution. The solutions for the orbital period of the exomoon candidate (Col. 3) show a comb-like structure owing to the discrete number of completed moon orbits that would fit a given value of the moon's initial orbital phase (Col. 4), which is essentially unconstrained. The moon-to-planet mass ratio (Col. 5) then shows a skewed normal distribution with a tail of large moon masses. Our results for the inclination $i_{\mathrm{s}}$ between the satellite orbit (around the planet) and the line of sight, and for the longitude of the ascending node of the moon orbit are shown in Cols. 6 and 7. The preference of $i_{\mathrm{s}}$ being either near 0 or near $\pm \pi$ (the latter is equivalent to a near-coplanar retrograde moon orbit) illustrates the well-known degeneracy of the prograde/retrograde solutions available from light curve analyses (Lewis and Fujii 2014, Heller and Albrecht 2014).

\subsubsection{Transit timing variations}

Next we consider the possibility of the transits being caused by a planet only. Neglecting the Hubble transit, our PTMCMC sampling of the three Kepler transits with our planetonly transit model gives an orbital period of $P=287.3776 \pm 0.0024 \mathrm{~d}$ and an initial 
3 Paper II: An alternative interpretation of the exomoon candidate signal in the combined Kepler and Hubble data of Kepler-1625

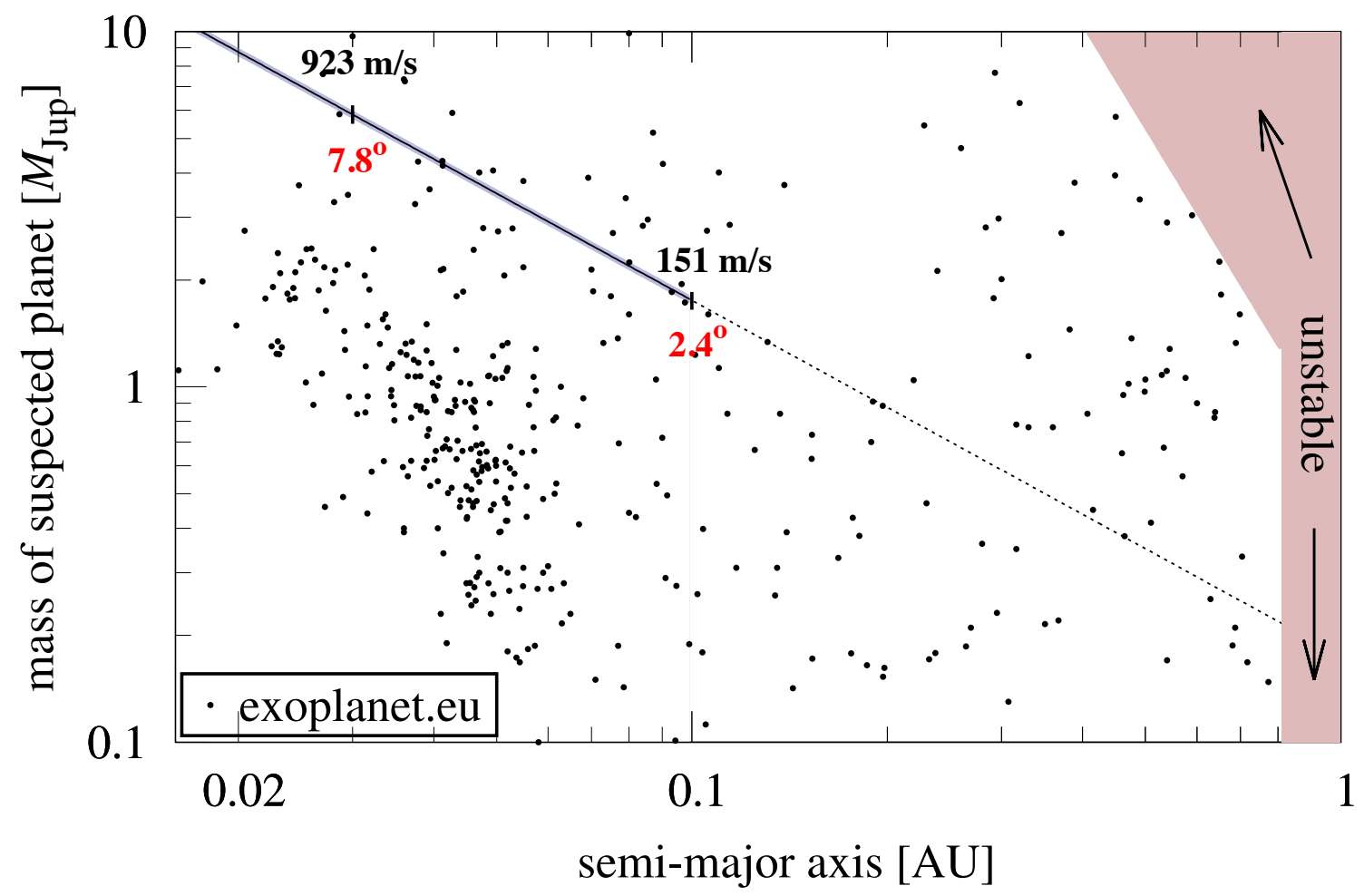

Figure 3.5: Mass estimate for the potential inner planet around Kepler-1625 based on the observed TTV of $73.728 \mathrm{~min}$. The thin pale blue fan around the solid curve shows the $1 \sigma$ tolerance fan of $\pm 2.016 \mathrm{~min}$. Values for semimajor axes $>0.1 \mathrm{AU}$ are poor approximations and thus shown with a dashed line. Black points show masses and semimajor axes of all planets from exoplanet.eu (as of 26 October 2018) around stars with masses between $0.75 M_{\odot}$ and $1.25 M_{\odot}$. A conservative estimate of a dynamically unstable region for the suspected inner planet, where its Hill sphere would touch the Hill sphere of Kepler-1625 b with an assumed mass of $1 M_{\mathrm{Jup}}$, is shaded in pale red. RV amplitudes and minimum orbital inclination with respect to Kepler- $1625 \mathrm{~b}$ are noted along the curve for the planetary mass estimate.

transit midpoint at $t_{0}=61.4528 \pm 0.0092 \mathrm{~d}$ in units of the Barycentric Kepler Julian Day (BKJD), which is equal to BJD - 2,454,833.0 d. The resulting transit time of the 2017 Hubble transit is $3222.6059 \pm 0.0182 \mathrm{~d}$.

Our planet-only model for the 2017 Hubble transit gives a transit midpoint at $3222.5547 \pm$ $0.0014 \mathrm{~d}$, which is $73.728( \pm 2.016)$ min earlier than the predicted transit midpoint. This is in agreement with the measurements of Teachey and Kipping (2018), who found that the Hubble transit occurred 77.8 min earlier than predicted. This observed early transit of Kepler-1625 b has a formal $\sim 3 \sigma$ significance. We note, however, that this $3 \sigma$ deviation is mostly dictated by the first transit observed with Kepler (see Fig. S12 in Teachey et al. 2018). We also note that this transit was preceded by a $\sim 1 \mathrm{~d}$ observational gap in the light curve, about $0.5 \mathrm{~d}$ prior to the transit, which might affect the local detrending of the data and the determination of the transit mid-point of a planet-only model. Moreover, with most of the TTV effect being due to the large deviation from the linear ephemeris of the 
first transit, stellar (or any other systematic) variability could have a large (but unknown) effect on the error bars that go into the calculations.

In Fig. 3.5 we show the mass of an unseen inner planet that is required to cause the observed 73.7 min TTV amplitude from our PTMCMC fit as a function of its unknown orbital semimajor axis. The mass drops from $5.8 M_{\text {Jup }}$ at $0.03 \mathrm{AU}$ to $1.8 \mathrm{M}_{\text {Jup }}$ at $0.1 \mathrm{AU}$. Values beyond 0.1 AU cannot be assumed to fulfill the approximations made for Eq. (3.3) and are therefore shown with a dashed line. The actual TTV amplitude of Kepler-1625 b could even be higher than the $\sim 73$ min that we determined for the Hubble transit, and thus the mass estimates shown for a possible unknown inner planet serve as lower boundaries.

The resulting radial velocity amplitudes of the star of $923 \mathrm{~m} \mathrm{~s}^{-1}$ (at $0.03 \mathrm{AU}$ ) and $151 \mathrm{~m} \mathrm{~s}^{-1}$ (at $0.1 \mathrm{AU}$ ), respectively, are indicated along the curve. Even if the approximations for a coplanar, close-in planet were not entirely fulfilled, our results suggest that RV observations of Kepler-1625 with a high-resolution spectrograph attached to a very large (8 $\mathrm{m}$ class) ground-based telescope could potentially reveal an unseen planet causing the observed TTV of Kepler-1625 b. Also shown along the curve in Fig. 3.5 are the respective minimum orbital inclinations (rounded mean values shown) between Kepler$1625 \mathrm{~b}$ and the suspected close-in planet required to prevent Kepler-1625 $\mathrm{b}$ from transiting the star. The exact values are $i=7.8_{-2.0}^{+1.1}$ degrees at $0.03 \mathrm{AU}$ and $i=2.4_{-0.6}^{+0.3}$ degrees at $0.1 \mathrm{AU}$.

The pale red shaded region is excluded from a dynamical point of view since this is where the planetary Hill spheres would overlap. The extent of this region is a conservative estimate because it assumes a mass of $1 M_{\text {Jup }}$ for Kepler-1625 b and neglects any chaotic effects induced by additional planets in the system or planet-planet cross tides etc. The true range of unstable orbits is probably larger. The black dots show all available exoplanet masses and semimajor axes from the Exoplanet Encyclopaedia, which illustrates that the suspected planet could be more massive than most of the known hot Jupiters.

\subsection{Conclusions}

With a $\triangle \mathrm{BIC}$ of -44.5 (using published Hubble data of Teachey and Kipping 2018) or -31.0 (using our own Hubble extraction and detrending) between the most likely planetonly model and the most likely planet-moon model, we find strong statistical evidence for a roughly Neptune-sized exomoon. In both cases of the data detrending, the most likely orbital solution of the planet-moon system, however, is very different from most of the other orbital realizations of our PTMCMC modeling and the most likely solutions do not seem to converge. In other words, the most likely solution appears to be an outlier in the distribution of possible solutions and small changes to the data can have great effects on the most likely orbital solution found for the planet-moon system. As an example, we find that the two different detrending methods that we explored produce different interpretations of the transit observed with Hubble: in one case our PTMCMC sampling finds the egress of the moon in the light curve, in the other case it does not (Fig. 3.2).

Moreover, the likelihood of this best-fit orbital solution is very different from the likelihoods of most other solutions from our PTMCMC modeling. We tested both a standard MCMC sampling and a parallel-tempering MCMC (Foreman-Mackey et al. 2013); the latter is supposed to explore both the parameter space at large and the tight peaks of the 
3 Paper II: An alternative interpretation of the exomoon candidate signal in the combined Kepler and Hubble data of Kepler-1625

likelihood function in detail. Our finding of the nonconvergence could imply that the likelihood function that best describes the data is non-Gaussian. Alternatively, with the BIC being an asymptotic criterion that requires a large sample size by definition (Stevenson et al.2012), our findings suggest that the available data volume is simply too small for the BIC to be formally applicable. We conclude that the $\triangle \mathrm{BIC}$ is an unreliable metric for an exomoon detection for this data set of only four transits and possibly for other data sets of Kepler as well.

One solution to evaluating whether the BIC or an alternative information criterion such as the Akaike information criterion (AIC; Akaike 1974) or the deviance information criterion (DIC; Spiegelhalter et al. 2002) is more suitable for assessing the likelihoods of a planet-only model and of a planet-moon model could be injection-retrieval experiments of synthetic transits (Heller et al. 2016b, Rodenbeck et al. 2018). Such an analysis, however, goes beyond the scope of this paper.

We also observe the TTV effect discovered by Teachey and Kipping (2018). If the early arrival of Kepler- 1625 b for its late-2017 transit was caused by an inner planet rather than by an exomoon, then the planet would be a super-Jovian mass hot Jupiter, the exact mass limit depending on the assumed orbital semimajor axis. For example, the resulting stellar radial velocity amplitude would be about $900 \mathrm{~m} \mathrm{~s}^{-1}$ for a $5.8 M_{\text {Jup }}$ planet at $0.03 \mathrm{AU}$ and about $150 \mathrm{~m} \mathrm{~s}^{-1}$ for a $1.8 M_{\text {Jup }}$ planet at $0.1 \mathrm{AU}$. From the absence of a transit signature of this hypothetical planet in the four years of Kepler data, we conclude that it would need to have an orbital inclination of at least $i=7.8_{-2.0}^{+1.1}$ (if it were at $0.03 \mathrm{AU}$ ) or $i=2.4_{-0.6}^{+0.3}$ degrees (if it were at $0.1 \mathrm{AU}$ ). If its inclination is not close to $90^{\circ}$, at which point its effect on the stellar RV amplitude would vanish, then the hypothesis of an unseen inner planet causing the Kepler-1625 TTV could be observationally testable.

Ground-based photometric observations are hardly practicable to answer the question of this exomoon candidate because continuous in- and near-transit monitoring of the target is required over at least two days. Current and near-future space-based exoplanet missions, on the other hand, will likely not be able to deliver the signal-to-noise ratios required to validate or reject the exomoon hypothesis. With a Gaia G-band magnitude of $m_{\mathrm{G}}=15.76$ (Gaia Collaboration et al. 2016, 2018) the star is rather faint in the visible regime of the electromagnetic spectrum and the possible moon transits are therefore beyond the sensitivity limits of the TESS, CHEOPS, and PLATO missions. 2MASS observations suggest that Kepler-1625 is somewhat brighter in the near-infrared (Cutri et al. 2003), such that the James Webb Space Telescope (launch currently scheduled for early 2021) should be able to detect the transit of the proposed Neptune-sized moon, for example via photometric time series obtained with the NIRCam imaging instrument.

All things combined, the fragility of the proposed photometric exomoon signature with respect to the detrending methods, the unknown systematics in both the Kepler and the Hubble data, the absence of a proper assessment of the stellar variability of Kepler1625 , the faintness of the star (and the resulting photometric noise floor), the previously stated coincidence of the proposed moon's properties with those of false positives (Rodenbeck et al. 2018), the existence of at least one plausible alternative explanation for the observed TTV effect of Kepler-1625 b, and the serious doubts that we have about the $\triangle \mathrm{BIC}$ as a reliable metric at least for this particular data set lead us to conclude that the proposed moon around Kepler-1625 b might not be real. We find that the exomoon hy- 
pothesis heavily relies on a chain of delicate assumptions, all of which need to be further investigated.

A similar point was raised by Teachey and Kipping (2018), and our analysis is an independent attempt to shed some light on the "unknown unknowns" referred to by the authors. For the time being, we take the position that the first exomoon has yet to be detected as the likelihood of an exomoon around Kepler-1625 b cannot be assessed with the methods used and data currently available.

\section{Acknowledgements}

The authors thank Kevin Stevenson, Hannah Wakeford and Megan Sosey for their help with the data analysis, and Nikole Lewis for the feedback on the manuscript. The authors would also like to thank the referee for a challenging and constructive report. This work was supported in part by the German space agency (Deutsches Zentrum für Luft- und Raumfahrt) under PLATO Data Center grant 50OO1501. This work made use of NASA's ADS Bibliographic Services and of the Exoplanet Encyclopaedia (http://exoplanet.eu). RH wrote the manuscript, proposed Figs. 3.2 - 3.4, generated Fig. 3.5, and guided the work. KR derived the star-planet-moon orbital simulations and the respective statistics and generated Figs. 3.2 - 3.4 GB performed the light curve extraction from the WFC3 Hubble data and generated Fig. 3.1. All authors contributed equally to the interpretation of the data. 



\section{Detection of Exomoons using Transit Variations}

\subsection{Observable Transit Variations}

Applying the full detection and characterization method used in Chapters 2 and 3 to many transiting exoplanets to find moons would be prohibitively expensive with respect to used computational resources and needed human oversight and interpretation.

Consequently we turned our interest to possible ways to detect exomoons using simpler and less resource-intensive methods. In the following I will describe a new method to possibly detect exomoons, derived from the already developed method of using transit timing and duration variation to search for exomoons.

Kipping (2009) proposes to use the variation of the midpoint and duration in the transits of a system to detect exomoons (called transit timing variation, TTV and transit duration variation, TDV). When searching for TTVs and TDVs, the duration and midpoint of each transit are fitted individually. The midpoint and duration of each transit is then compared to the midpoint and duration from a global fit over all transits in the light curve.

The idea of TTVs and TDVs as exomoon indicator is based on the idea that the planet moves periodically around the planet-moon barycenter. Specifically, the TTV and TDV variations are phase-shifted to each other, which leads Heller et al. (2016b) to suggest to look for an ellipse-like pattern in the TTV-TDV space of a system. However, this approach ignores the contribution of the moon transit to the total light curve. The moon transit generally has a different midpoint and duration compared to the planet transit, stemming from the orbital orientation of the two bodies. If the planet and moon transit overlap, this leads to an overall transit shape that can not be described by the planet-only transit model. When trying to fit that light curve with the planet-only model, one will generally not recover the true midpoint and duration of the planet transit, but slightly different ones.

Szabó et al. (2006) explore the effect a moon transit has on the photometric center of the combined transit, which is defined as $\sum_{i} t_{i} \Delta m_{i} / \sum_{i} \Delta m_{i}$, where $t_{i}$ are the times at which the light curve is measured and $\Delta m_{i}$ the relative dimming of the light curve at that time.

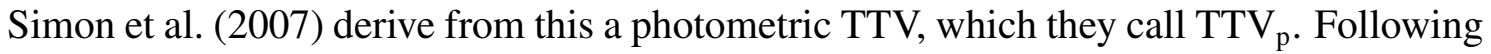
that notation, we refer to the TTV and TDV due to the movement around the planet-moon barycenter as $\mathrm{TTV}_{\mathrm{b}}$ and $\mathrm{TDV}_{\mathrm{b}}$ and the TTV and TDV due to the existence of a moon transit as $\mathrm{TTV}_{\mathrm{p}}$ and $\mathrm{TDV}_{\mathrm{p}}$.

If the planet and moon transits are close together, the depth of the transit also changes. If the planet-only model is fitted to the light curve, this results in a larger fitted planet- 
to-star radius ratio. We refer to the variation of this quantity as the planetary radius variation, PRV.

\subsection{Transit Variations for Different Moon Phases}

To test this change in the TTV, TDV and PRV, I generate light curves with a single transit of a Jupiter-sized planet and moon of variable size in a $30 \mathrm{~d}$ orbit around a sun-like star. The moon's period is $P_{\mathrm{s}}=3.55 \mathrm{~d}$, the same as Europa's period around Jupiter. I let the moon phase vary from 0 to 1 . For each moon phase I fit a planet-only model to the light curve, where only the radius ratio $r$, the transit duration $t_{\mathrm{T}}$ and the transit midpoint $t_{\text {mid }}$ are allowed to vary. The other parameters (impact parameter, period and limb darkening parameters) are kept constant. This is meant to simulate a situation where the non-changing parameters can be found by fitting the transit model to the global light curve with multiple transits.

To distinguish between the barycentric and photometric variations, we test three different combinations of moon mass and radius. To only retain the barycentric effect, I set the moon radius to 0 and the moon mass to $1 M_{\oplus}$, for the photometric effect I set the moon mass to 0 and the moon radius to $1 R_{\oplus}$. Finally, for the combined effect, I set the moon radius to $1 R_{\oplus}$ and the moon mass to $1 M_{\oplus}$. I repeat this test for two different moon inclinations $i_{\mathrm{s}}=0$ and $i_{\mathrm{s}}=0.2$.

Fig. 4.1 shows the results of this test. The barycentric component of the TTV and TDV show the sinusoidal variations expected from the circular orbit, and form the an ellipse in the TTV-TDV space (panel $d$ )) as described in Heller et al. (2016b). However, the photometric component of the TTV and TDV signals have roughly the same amplitude as the barycentric component, but have a more complicated shape.

The photometric component of the TTV signal has almost a constant amplitude of $20 \mathrm{~s}$ for most of the moon phases except when the moon aligns with the planet. The sign of the photometric TTV signal changes depending on whether the moon transit takes place before or after the planetary transit. The photometric TTV shifts the transit in the opposite direction compared to the barycentric one. This leads to diminished amplitude of the total TTV signal. The inclination has only an effect when planet and moon are close conjunction and a occultation occurs at low inclinations.

Outside of conjunctions, the photometric TDV signal depends only on the time difference between planet transit and moon transit. The further apart planet and moon are, the larger the $\operatorname{TDV}_{\mathrm{p}}$ signal is. During conjunctions the transit duration is lowered if the moon overtakes the planet and raised if the planet overtakes the moon. The occurrence of a occultation has a strong effect on the TDV $\mathrm{p}_{\mathrm{p}}$ signal. The TTV-TDV ellipse in TTV-TDV space becomes much more complicated if the photometric TTV and TDV components are taken into account. The figure traced by the total TTV and TDV signal no longer has any recognizable features and will also depend on the relative strength of the photometric and barycentric components.

The PRV signal has only a very small barycentric component, and it only manifests if the moon is inclined. It is caused by the movement of the planet around the planetmoon barycenter changing the planet's impact parameter. Due to the limb-darkening the planet blocks less light if the impact parameter is higher and appears to have a smaller 

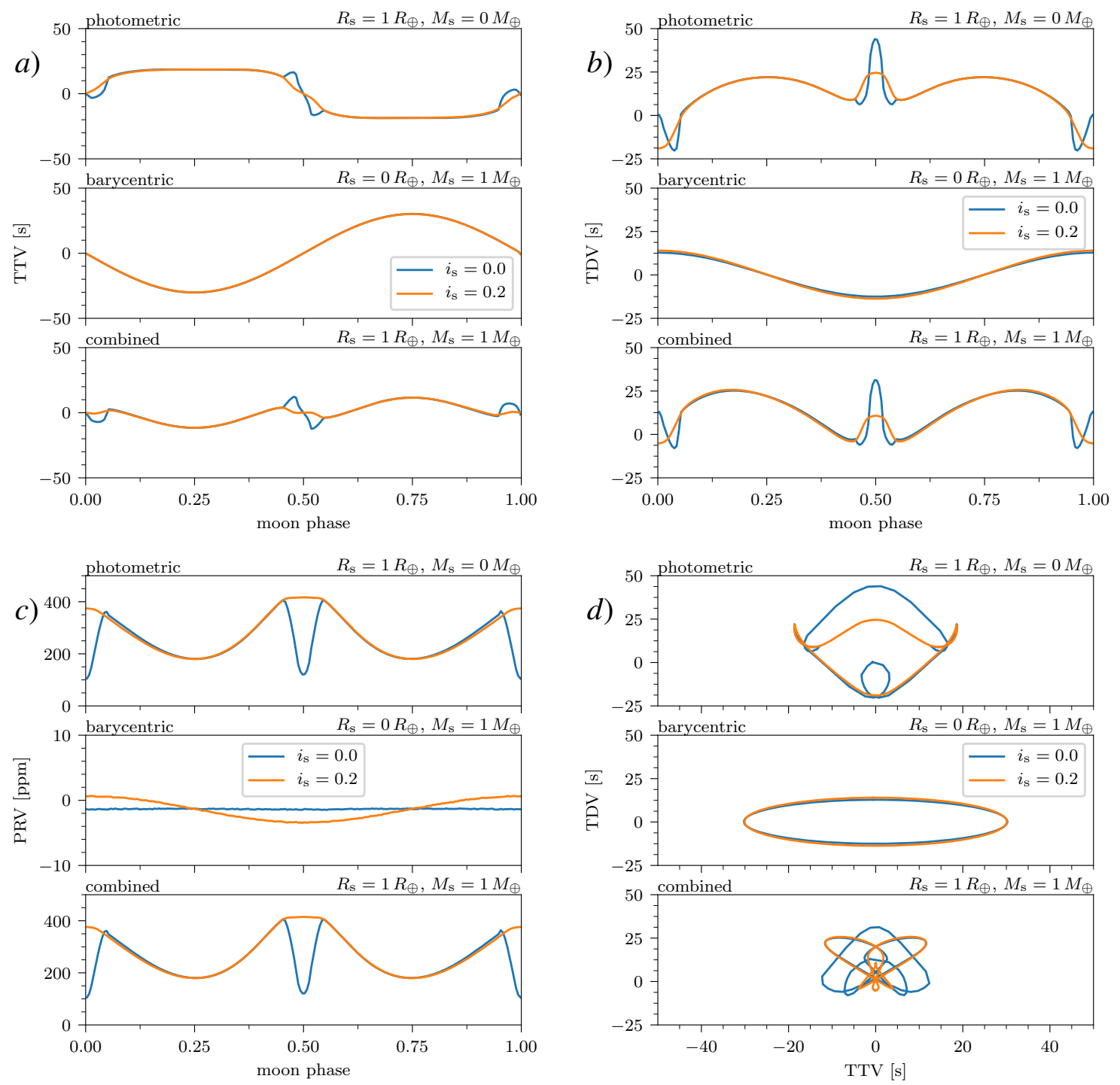

Figure 4.1: Transit parameter variation due to the presence of a Earth-sized moon in $3.55 \mathrm{~d}$ orbit around a Jupiter-sized planet in a $30 \mathrm{~d}$ orbit around its star. We separate the effect due to the planet orbiting the planet-moon barycenter and the effect of the presence of the moon transit by setting the moon radius to 0 (top of each panel) or the moon mass to 0 (center of each panel). The bottom of each panel shows the combined effect. Panel $a$ ) shows the TTV signal, panel $b$ ) the TDV signal and panel $c$ ) the PRV signal for different moon phases. Panel $d$ ) shows the TTV and TDV signal in TTV-TDV space. 
radius. The PRV signal of the photometric component depends on the time difference between planet transit and moon transit. When planet and moon are in conjunction, but inclined such that no occultation occurs, the two transit curves completely overlap and form an "effective" transit curve where the blocked area is the combined blocked area of the two bodies. For the used planet-moon system, the expected PRV signal would then be $\left(\sqrt{R_{\mathrm{p}}^{2}+R_{\mathrm{s}}^{2}}-R_{\mathrm{p}}\right) / R_{\star}=408 \mathrm{ppm}$, which is the amplitude we observe in Fig. 4.1 . When planet and moon are separated further, this amplitude drops. If a occultation occurs during transit, the total area where light is blocked is smaller, meaning that the PRV is also smaller.

\subsection{Transit Variations in Observed Transits}

Next, we look at the TTV, TDV and PRV signal in light curves with a series of transits. I generate a synthetic light curve with a length of 2 years. At a planetary orbit of $30 \mathrm{~d}$, this means there are 24 transits in the total light curve. For each of the transits I fit a planetonly model as described above. Fig. 4.2 shows two cases: In one, no noise is added to the generated light curve and the cadence (how often the light curve is measured) is
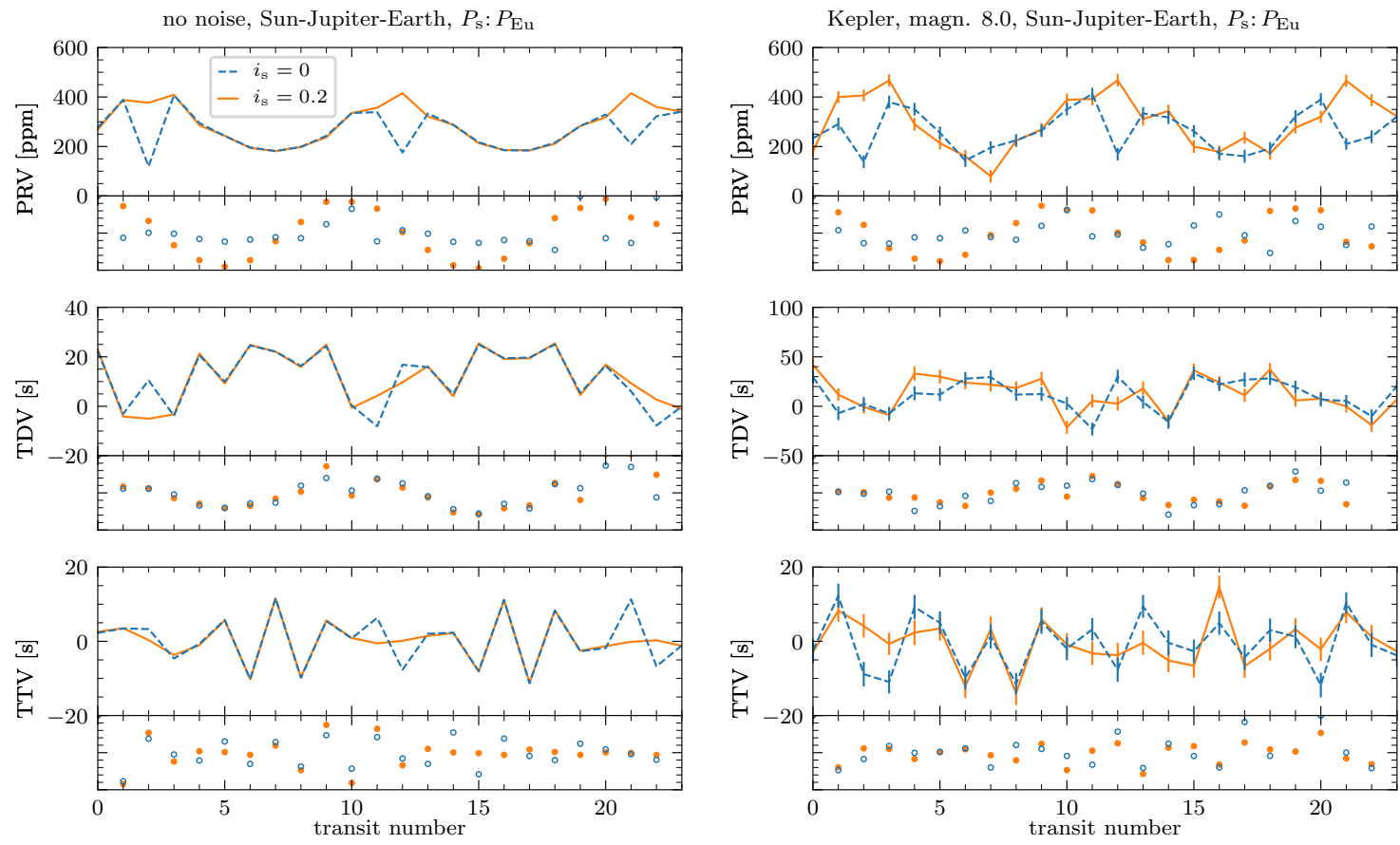

Figure 4.2: PRV, TDV and TTV of a series of transits in a simulated light curve over a time of 2 years. An Earth-sized moon orbits its Jupiter-sized planet in a $3.55 \mathrm{~d}$ orbit. The planets orbit around the sun-like star is $30 \mathrm{~d}$ for a total of 24 transits. The moon has either no inclination $\left(i_{\mathrm{s}}=0\right)$ or a slight inclination to avoid planet-moon occultation $\left(i_{\mathrm{s}}=0\right)$. For each of the three indicators the time series and its autocorrelation is shown. Left: No noise is added to the light curve. The cadence of observation is $25 \mathrm{~s}$. Right: Noise corresponding to a $m_{V}=8$ sun-like star observed with the Kepler space telescope at a cadence of $29.4 \mathrm{~min}$. 
set to $25 \mathrm{~s}$. In the other case, an observation of a sun-like star with apparent magnitude $m_{V}=8$ using the Kepler space telescope is simulated. The cadence is $29.4 \mathrm{~min}$, photon and instrumental noise is added as white noise with an amplitude of 9 ppm (Koch et al. 2010). Additionally, granulation noise is added according to the model in Gilliland et al. (2011), which models the granulation power spectrum.

Measuring the TTV, TDV and PRV signal in the case of no noise reveals one additional complication compared to looking at the variation with the moon phase: The moon orbits its planet multiple times from one transit to the next. This means that the moon phase at the next transit can be completely different from the last one, depending on the exact ratio of the barycenter orbit period and the moon orbit period $P_{\mathrm{b}} / P_{\mathrm{s}}$. If we write this ratio as the sum of an integer $n$ and the remainder $r, P_{\mathrm{b}} / P_{\mathrm{s}}=n+r$, it becomes clear that the moon phase advances by $r$ from transit to transit. For the used system, $r=0.45$, meaning the moon will advance a bit less than half an orbit every transit.

The means that we do not recover the phase-dependent TTV, TDV, and PRV signal, but an aliased version thereof, as can be seen very clearly in the noise-less TTV signal. The TTV and TDV signals are barely visible in the case of added noise, however the autocorrelation of the TDV signal still shows a sine-like variation. The PRV signal is clearly visible, independently of whether an occultation occurs or not. Its autocorrelation indicates a periodicity in the signal around every 10 transits.

In summary, we find that the previously proposed transit timing and duration variation might not be reliable indicators for the presence of an exomoon. We do propose the planetary radius variation as an additional exomoon indicator. The biggest source of false positive exomoon detections using the PRV indicator would most likely be long term stellar variability, e.g. caused by stellar rotation. 



\section{Discussion}

In the following I will discuss the results from Chapters 2, 3 and 4. I also discuss two topics that are not touched upon in the chapters that form the core of this thesis, but are nevertheless worthwhile to consider in the context of hunting exomoons: I discuss the interplay between star, planet and moon parameters and how a global model to determine all of them at the same time can be useful. I also shortly give an outlook how machine learning might be used as a possible tool for exomoon detection.

\subsection{The Exomoon Candidate around Kepler-1625 b}

One of the key findings of Rodenbeck et al. (2018) (Chapter 2) is a roughly 10-20\% false positive rate in the detection of exomoons when we inject artificial planet and moon transits into real light curves of Kepler-1625, compared to a $40 \%$ true positive rate (see Sec. 2.3.2), suggesting a better than even chance to have found a genuine moon once a detection has been made. This however neglects the occurrence rate of such massive moons. It stands to reason that massive moons are rare, otherwise the first detection of an exomoon would probably have happened around one of the brighter stars observed by Kepler. With a low occurrence rate of massive moons a false positive rate of $10 \%$ would produce much more false positives in total than genuine detections with a true positive rate of $40 \%$. For smaller moons the ratio of false positive rate and true positive rate will likely be larger for a given system.

In Sec. 2.2.4.2 we also test our exomoon detection/characterization method on synthetic data using white noise at different noise levels. This test shows no false positives when a planet without moon is injected. This implies that the false positives in the injection-retrieval experiment are due to the detrending methods used, non-white noise components in the light curve or both. A detailed modeling of the Kepler-1625 light curve might be able to reduce the false positive rate and simultaneously lower the overall noise level of the light curve. For some of the synthetic light curves in the injection-retrieval experiment, the classification whether it contains a moon depends on the detrending method used, which also points towards the detrending being one of the possible sources for false positives.

We find in Heller et al. (2019) (Chapter 3) that the evidence in hugely in favor of the one-moon hypothesis. However, there are multiple points that leads us to doubt these results. Teachey and Kipping (2018) describe that the evidence for a moon using only Kepler photometry almost vanishes compared to the results in Teachey et al. (2018) due to changes in the Kepler light curve extraction algorithm. Our own best-fit model light curves also show a different moon signature compared to the best-fitting ones in Teachey 
and Kipping (2018) for the Kepler transits, both when using the light curve where we detrended the Hubble data ourselves and the detrended light curve provided by Teachey and Kipping (2018). This suggests that the potential moon signatures in the Kepler light curve only play a marginal role in determining the likelihood of the presence of the moon.

This leaves two main arguments pointing towards the existence of the moon: The dip in the Hubble light curve after the main transit and that the Hubble main transit is 78 min earlier than predicted from the Kepler transits. The possible moon transit in the Hubble data is cut short by the end of the Hubble observations. In fact, the best-fit model light curves in Fig. 3.2 show two different possibilities depending on the how the Hubble light curve is detrended. In one case, the moon transit ends just as the Hubble observation ends, in the other the moon transit is still ongoing at the end of observation. Teachey and Kipping (2018) observe a similar effect for different detrending methods. It is not clear from the Hubble light curve if the flux level returns to a higher level than during the moon transit afterwards.

The TTV of 78 min might have been caused by a second planet closer to the star, as described in Sec. 3.3.2. Radial Velocity observations in the future might be able to tell if a second planet is present and responsible for the observed TTV.

Given the work and analysis needed to validate or refute a single candidate (Teachey et al. 2018, Teachey and Kipping 2018, Rodenbeck et al. 2018, Heller et al. 2019), a large number of false positives would be crippling to any effort to find and validate more candidates.

\subsection{Transit Variations as Exomoon Indicators}

The ratio of the barycentric and photometric contributions of the TTV signal depends on the density of the moon: At low densities, the photometric component is stronger, at high densities the barycentric component. For moons with terrestrial densities (3 $5 \mathrm{~g} \mathrm{~cm}^{-3}$ ), the components almost cancel out (see Fig. 4.1 (a)) and the TTV signal caused by an exomoon might be very hard to observe. In contrast to the TTV and TDV signal, the PRV signal is always dominated by the photometric component. This means that it always retains the same shape, independent of the moon's density, although, of course, the amplitude depends on the moon's radius.

If the inclination of the moon is such that occultations can happen during the transit, the observed TTV, TDV and PRV signal gets a lot more complicated since an occultation changes the TTV, TDV and PRV signal on an amplitude comparable to the changes outside of an occultation, but only in a very small regions of the moon phase. For example, the PRV signal changes from an almost sine-like signal to a more complex signal. These more complex shapes make the detection of the variations in an automated way harder.

The existence of the periodic TTV, TDV and PRV signals become only visible once at least one or two full variation cycles have been observed. This means that this new exomoon indicator works best in the context of long term transit searches such as Kepler and PLATO.

Localized features on a star's surface such as spots or faculae can also cause variations in the measured exoplanet radius. Hence, any search for moon-induced PRVs needs to 


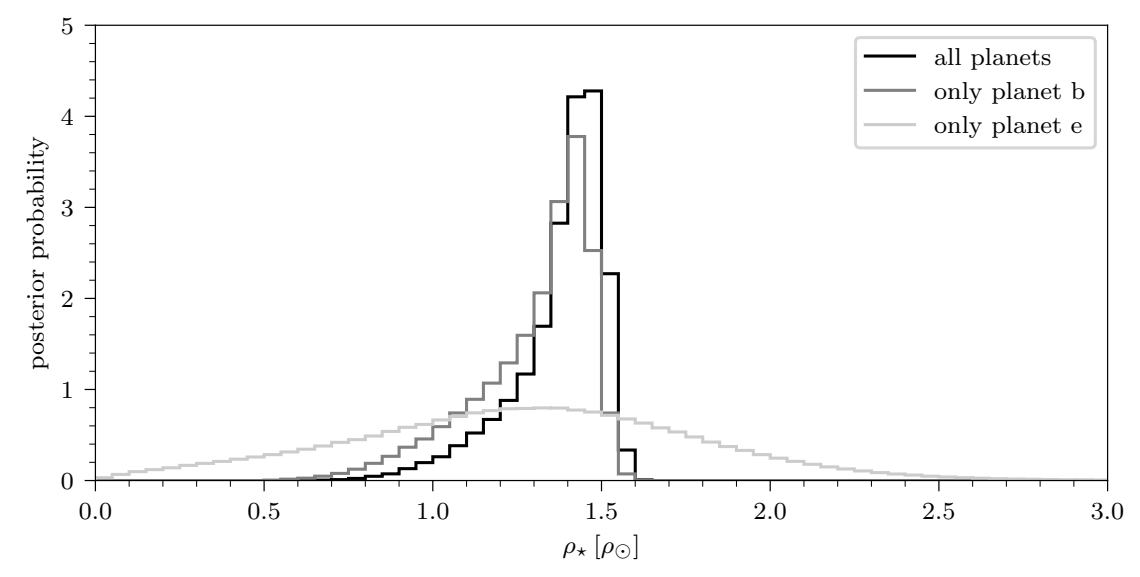

Figure 5.1: Posterior probability of the stellar density of K2-32. The posterior probability was obtained using an MCMC algorithm fitting the star's entire K2 light curve with the transits of either all four planets (black), the biggest planet (dark gray) or only the new planet (light gray). The stellar density is mostly constrained by the transits of the biggest planet, however the the combined fit is still better. The transits of the new planet constrain the stellar density only lightly.

include the analysis of stellar variations and verify that any candidate signals are not caused by the star.

\subsection{The Star-Planet-Moon Connection}

The determination of stellar, planetary and moon parameters are interconnected. By fitting the transit shape of a planet we can for example calculate its star's maximum density (see e.g. Seager and Mallén-Ornelas 2003). In the other direction, accurate values for the star's mass and radius from other sources help constrain the transit parameters, mostly by allowing for a better fit of the impact parameter.

In an upcoming work ${ }^{1}$ I use the fact that there are multiple planets with transits in the system to determine the stellar density of K2-32. For a system with only one transiting planet, the stellar density (which can be used as an alternative to $a / R_{\star}$ in a transit fit) is normally strongly correlated to the impact parameter. However, in a system of multiple transiting systems, the different transit shapes help in constraining the stellar density (see Fig. 5.1). The well-defined stellar density in turn helps to constrain the maximum transit duration for a given period and consequently, the impact parameter of the planets and the radius ratios of the planets.

A combined fit of exoplanet and exomoon parameters can also help in determining the mass of the planet and moon, which is not possible with transit fits of a planet only. Kipping (2010) gives an expression for the planet and moon's density in terms of the observed transit parameters, which can be applied to the parameter we found for Kepler-1625 b. Us-

\footnotetext{
${ }^{1}$ The Transit Least Squares Survey I. Discovery and validation of an Earth-sized planet in the four-planet system K2-32 near the 1:2:5:7 resonance: Heller, R, Rodenbeck, K, Hippke, M, submitted to A\&A. We use the Transit Least Squares algorithm described in Hippke and Heller (2019) to detect a small exoplanet that has been missed by the Box Least Squares algorithm and characterize it.
} 

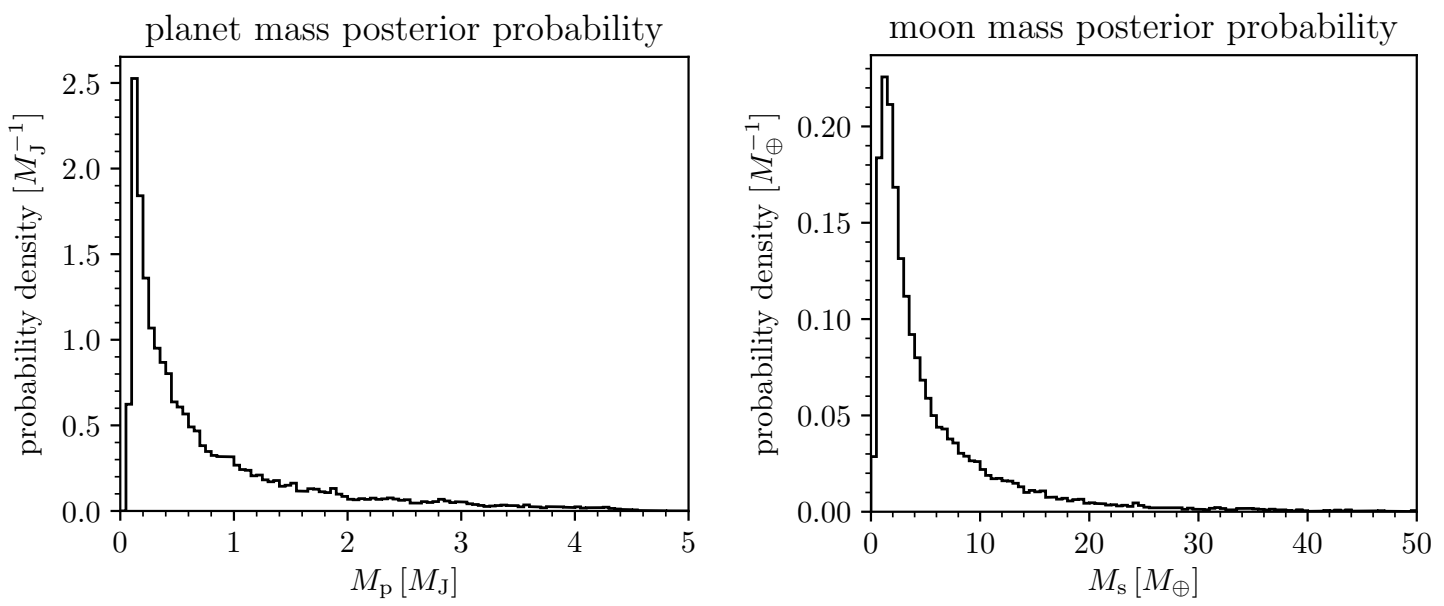

Figure 5.2: The mass distribution of the exoplanet Kepler-1625 b (left) and of its moon candidate (right). The distribution is calculated from the posterior parameter distribution of the MCMC run in Chapter 3 .

ing a stellar radius of 1.793 (Mathur et al.2017), this gives posterior distributions for both the planet and moon masses (see Fig. 5.2). The moon's mass is with 95\% confidence smaller than $19.3 M_{\oplus}$, which is in agreement with a Neptune-sized body. However, the distribution is heavily skewed towards very light moons and planets, which raises questions about the physical plausibility of those solutions. The planet mass $M_{\mathrm{p}}=0.44_{-0.30}^{+1.34} M_{\mathrm{J}}$ is also not very well constrained. Extrapolating from already observed exoplanets, a gas giant with the radius of Kepler-1625 $\mathrm{b}$ is expected to have a mass between 0.15 and $6.3 M_{\mathrm{J}}$ (Howard 2013), our result for the mass of Kepler-1625 b is within the expected region. Using the parameter set with the maximum likelihood, the planet mass is $M_{\mathrm{p}}=3 M_{\mathrm{J}}$ and the moon mass $M_{\mathrm{s}}=13 M_{\oplus}$. This would cause a maximum TTV signal of around $20 \mathrm{~min}$ (Kipping 2009), which is smaller than the 78 min TTV discussed by Teachey and Kipping (2018).

\subsection{Exomoon Detection using Machine Learning}

Machine learning has shown great potential in the last few years to quickly and efficiently sort and classify all kinds of data. Machine learning could be used to find possible moon candidates in a large number of transit light curves. To test the viability of this approach, we test machine learning ${ }^{2}$ on a simple test case: A planet-moon system similar to Kepler$1625 \mathrm{~b}$, with white noise added.

I generate data sets where two parameter are varied: the radius of the moon $R_{\mathrm{S}}$ and the amplitude of the white noise we add on top of the light curve. I use a modified version of the model used in Rodenbeck et al. (2018) to generate the transit light curves, either with

\footnotetext{
${ }^{2}$ The following section describes the results of the article Detectability of Exomoon Candidate Signals Using 1D ConvNet with Total Variation Loss by Alshehhi, R., Rodenbeck, K., Gizon, L. (2019), in prep. LG proposed the research idea. RA proposed the the Machine Learning algorithm and analyzed the data and the results. KR prepared the simulated data and the BIC analysis. All authors contributed to the final manuscript.
} 


\begin{tabular}{r|l} 
parameter & value \\
\hline star radius $R_{\star}$ & $1 R_{\odot}$ \\
star mass $M_{\star}$ & $1 M_{\odot}$ \\
limb-darkening (quadratic) & $q_{1}=0.6, q_{2}=0.4$ \\
planet radius $R_{\mathrm{p}}$ & $12 R_{\oplus}$ \\
planet mass $M_{\mathrm{p}}$ & $318 M_{\oplus}$ \\
barycenter period $P_{\mathrm{b}}$ & 287 day \\
moon radius $R_{\mathrm{s}}$ & $0.25-4.75 R_{\oplus}$ \\
moon density $\rho_{\mathrm{s}}$ & $1 \rho_{\oplus}$ \\
moon semi-major axis $a_{\mathrm{ps}}$ & $20 R_{\mathrm{J}}$ \\
moon phase $\varphi_{\mathrm{s}}$ & $0-1$ (random)
\end{tabular}

Table 5.1: Star, planet and moon parameters used to generate the data sets of light curves for machine learning training and testing. The orbital configuration is close to that of Kepler-1625 b, around which an exomoon candidate signal was found (Teachey et al. 2018, Teachey and Kipping 2018). The quadratic limb-darkening model uses the parametrization from Kipping (2013).

a moon or without a moon. The moon's orbit is not inclined relative to the observer, and planet-moon occultation was added to the model. For the parameters used see Table 5.1 .

In one data set the moon radius is varied between 0.25 and $4.75 R_{\oplus}$, while the noise level is fixed at $100 \mathrm{ppm}$. The moon radius is varied in steps of $0.5 R_{\oplus}$. For each radius 100000 realizations of the light curve are generated. The added noise is a different realization for each light curve. The moon phase is chosen randomly for each realization. 1000000 light curves without a moon are generated, equal to the total number of light curves with a moon in this data set.

In a second data set, the moon radius is fixed at $1 R_{\oplus}$, while the noise level is varied from $25 \mathrm{ppm}$ to $475 \mathrm{ppm}$ insteps of $50 \mathrm{ppm}$. As in the first data set, the noise and moon phase is newly draw for each realization. For each noise level 100000 light curves with moons are generated, and 1000000 light curves without a moon, but with different noise levels are generated.

Each subset (each radius and noise level) is divided into two set: the training set and the test set. For the machine learning a Convolution Neural Network (ConvNet, Krizhevsky et al. (2012), Liu and Deng (2015)), implemented by Rasha Alshehhi, is used. The ConvNet is trained on the training set and evaluated on the test set. I perform an analysis similar to the one in Rodenbeck et al. (2018) in which I try to determine if a moon is present or not on 100 light curves of each test set. I use the $\triangle \mathrm{BIC}$ (with a threshold of 0 ) to decide if a moon is present or not.

The light curves are classified into four categories: True positives, when the algorithm correctly decides that a moon is present for a light curve containing a moon, true negatives, when the algorithm correctly decides that no moon is present, false positives, when the algorithm incorrectly finds a moon in a light curve containing no moon and false negatives, when the algorithm incorrectly decides no moon is present even if the light curve contains a moon.

Fig. 5.3 shows the resulting performance metrics of the ConvNet analysis and my own "classic" analysis. Both methods have no problem detecting very large moons or an 

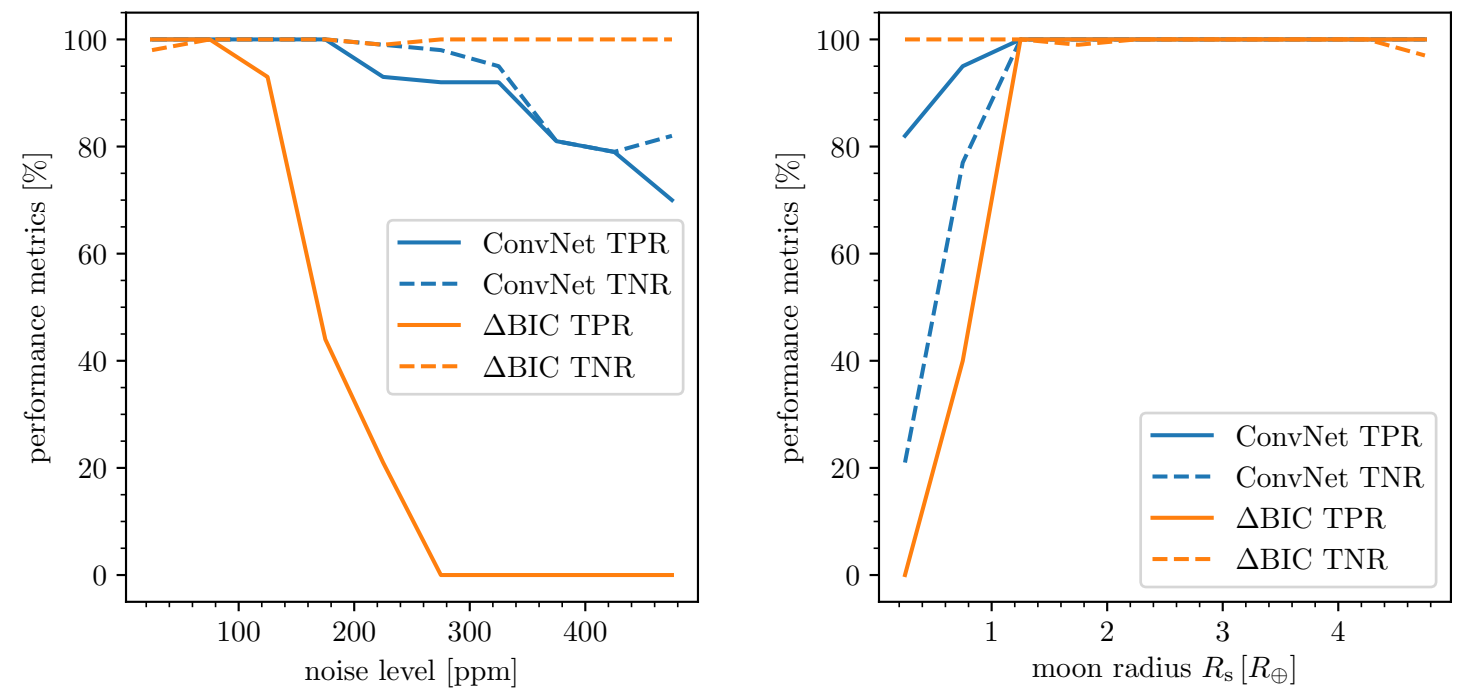

Figure 5.3: Performance metrics of the ConvNet algorithm (blue) and my own analysis using the $\triangle \mathrm{BIC}$ (orange). Left: The noise level is varied from 25 to $475 \mathrm{ppm}$, while the moon radius is kept constant at $1 R_{\oplus}$. Right: The moon radius is varied from 0.25 to $4.75 R_{\oplus}$ while the noise level is kept constant at $100 \mathrm{ppm}$. For the two methods in each panel the true positive rate (TPR, solid line) and the true negative rate (TNR, dashed line) is shown.

Earth-sized moon at very low noise levels. My own analysis overall is very conservative for larger noise levels and smaller moons. The true negative rate stays constantly close to $100 \%$, while the true positive rate drops to $0 \%$ at a noise level of $275 \mathrm{ppm}$ and a moon radius of $0.25 R_{\oplus}$. The ConvNet algorithm shows a very good performance even for high noise levels. For a noise level between 350 and $500 \mathrm{ppm}$ the true positive rate and true negative rate is still at 70-90\%.

\subsection{Outlook}

Kepler-1625 b's orbit only lasts three-quarters of a year. This means that transits of this exoplanet can be observed quite frequently, with the next transit being in May 2019. It will be interesting to see if more observed transits will shift the probabilities for or against a moon.

In the previous section I described a first attempt to use machine learning as a tool to detect exomoons. We only tested machine learning in a simple setup, with only one planet/moon configuration and white noise. To apply exomoon detection using machine learning on real data, the machine learning setup would need to be trained and tested with different noise types and generalized to more system configurations (different orbit configurations, planet sizes, number of observed transits). 


\section{Bibliography}

Agnor, C. B. and Hamilton, D. P. Neptune's capture of its moon Triton in a binary-planet gravitational encounter. Nature, 441:192-194, May 2006. doi: 10.1038/nature04792.

Agol, E., Steffen, J., Sari, R., and Clarkson, W. On detecting terrestrial planets with timing of giant planet transits. MNRAS, 359:567-579, May 2005. doi: 10.1111/j.1365-2966. 2005.08922.x.

Agol, E., Jansen, T., Lacy, B., Robinson, T. D., and Meadows, V. The Center of Light: Spectroastrometric Detection of Exomoons. ApJ, 812:5, October 2015. doi: 10.1088/ 0004-637X/812/1/5.

Aigrain, S. and Irwin, M. Practical planet prospecting. MNRAS, 350:331-345, May 2004. doi: 10.1111/j.1365-2966.2004.07657.x.

Aigrain, S., Hodgkin, S. T., Irwin, M. J., Lewis, J. R., and Roberts, S. J. Precise time series photometry for the Kepler-2.0 mission. MNRAS, 447:2880-2893, March 2015. doi: 10.1093/mnras/stu2638.

Aigrain, S., Parviainen, H., and Pope, B. J. S. K2SC: flexible systematics correction and detrending of $\mathrm{K} 2$ light curves using Gaussian process regression. MNRAS, 459: 2408-2419, July 2016. doi: 10.1093/mnras/stw706.

Aizawa, M., Uehara, S., Masuda, K., Kawahara, H., and Suto, Y. Toward Detection of Exoplanetary Rings via Transit Photometry: Methodology and a Possible Candidate. $A J$, 153:193, April 2017. doi: 10.3847/1538-3881/aa6336.

Akaike, H. A New Look at the Statistical Model Identification. IEEE Transactions on Automatic Control, 19:716-723, 1974.

Baker, S. and Matthews, I. Equivalence and effciency of image alignment algorithms. Proceedings of the 2001 IEEE Conference on Computer Vision and Pattern Recognition, Volume 1, Pages 1090-1097, December 2001.

Ballard, S., Fabrycky, D., Fressin, F., Charbonneau, D., Desert, J.-M., Torres, G., Marcy, G., Burke, C. J., Isaacson, H., Henze, C., Steffen, J. H., Ciardi, D. R., Howell, S. B., Cochran, W. D., Endl, M., Bryson, S. T., Rowe, J. F., Holman, M. J., Lissauer, J. J., Jenkins, J. M., Still, M., Ford, E. B., Christiansen, J. L., Middour, C. K., Haas, M. R., Li, J., Hall, J. R., McCauliff, S., Batalha, N. M., Koch, D. G., and 
Borucki, W. J. The Kepler-19 System: A Transiting $2.2 \mathrm{R}_{\oplus}$ Planet and a Second Planet Detected via Transit Timing Variations. ApJ, 743:200, Dec 2011. doi: 10.1088/0004-637X/743/2/200.

Barnes, J. W. Effects of Orbital Eccentricity on Extrasolar Planet Transit Detectability and Light Curves. Publications of the Astronomical Society of the Pacific, 119:986993, September 2007. doi: 10.1086/522039.

Ben-Jaffel, L. and Ballester, G. E. Transit of exomoon plasma tori: New diagnosis. ApJ, 785(2):L30, 2014.

Berta, Z. K., Charbonneau, D., Désert, J.-M., Miller-Ricci Kempton, E., McCullough, P. R., Burke, C. J., Fortney, J. J., Irwin, J., Nutzman, P., and Homeier, D. The Flat Transmission Spectrum of the Super-Earth GJ1214b from Wide Field Camera 3 on the Hubble Space Telescope. ApJ, 747:35, March 2012. doi: 10.1088/0004-637X/747/ $1 / 35$.

Borucki, W. J., Koch, D., Basri, G., Batalha, N., Brown, T., Caldwell, D., Caldwell, J., Christensen-Dalsgaard, J., Cochran, W. D., DeVore, E., Dunham, E. W., Dupree, A. K., Gautier, T. N., Geary, J. C., Gilliland, R., Gould, A., Howell, S. B., Jenkins, J. M., Kondo, Y., Latham, D. W., Marcy, G. W., Meibom, S., Kjeldsen, H., Lissauer, J. J., Monet, D. G., Morrison, D., Sasselov, D., Tarter, J., Boss, A., Brownlee, D., Owen, T., Buzasi, D., Charbonneau, D., Doyle, L., Fortney, J., Ford, E. B., Holman, M. J., Seager, S., Steffen, J. H., Welsh, W. F., Rowe, J., Anderson, H., Buchhave, L., Ciardi, D., Walkowicz, L., Sherry, W., Horch, E., Isaacson, H., Everett, M. E., Fischer, D., Torres, G., Johnson, J. A., Endl, M., MacQueen, P., Bryson, S. T., Dotson, J., Haas, M., Kolodziejczak, J., Van Cleve, J., Chandrasekaran, H., Twicken, J. D., Quintana, E. V., Clarke, B. D., Allen, C., Li, J., Wu, H., Tenenbaum, P., Verner, E., Bruhweiler, F., Barnes, J., and Prsa, A. Kepler Planet-Detection Mission: Introduction and First Results. Science, 327:977, February 2010. doi: 10.1126/science.1185402.

Broeg, C., Fortier, A., Ehrenreich, D., Alibert, Y., Baumjohann, W., Benz, W., Deleuil, M., Gillon, M., Ivanov, A., Liseau, R., Meyer, M., Oloffson, G., Pagano, I., Piotto, G., Pollacco, D., Queloz, D., Ragazzoni, R., Renotte, E., Steller, M., and Thomas, N. CHEOPS: A transit photometry mission for ESA's small mission programme. In European Physical Journal Web of Conferences, volume 47 of European Physical Journal Web of Conferences, page 03005, April 2013. doi: 10.1051/epjconf/20134703005.

Brown, T. M., Charbonneau, D., Gilliland, R. L., Noyes, R. W., and Burrows, A. Hubble Space Telescope Time-Series Photometry of the Transiting Planet of HD 209458. ApJ, 552:699-709, May 2001. doi: 10.1086/320580.

Cabrera, J. and Schneider, J. Detecting companions to extrasolar planets using mutual events. AEFA, 464(3):1133-1138, 2007. doi: 10.1051/0004-6361:20066111.

Cameron, A. G. W. and Ward, W. R. The Origin of the Moon. In Lunar and Planetary Science Conference, volume 7 of Lunar and Planetary Inst. Technical Report, March 1976. 
Canup, R. M. and Ward, W. R. Formation of the Galilean Satellites: Conditions of Accretion. AJ, 124:3404-3423, December 2002. doi: 10.1086/344684.

Coustenis, A., Achterberg, R. K., Conrath, B. J., Jennings, D. E., Marten, A., Gautier, D., Nixon, C. A., Flasar, F. M., Teanby, N. A., Bézard, B., Samuelson, R. E., Carlson, R. C., Lellouch, E., Bjoraker, G. L., Romani, P. N., Taylor, F. W., Irwin, P. G. J., Fouchet, T., Hubert, A., Orton, G. S., Kunde, V. G., Vinatier, S., Mondellini, J., Abbas, M. M., and Courtin, R. The composition of Titan's stratosphere from Cassini/CIRS mid-infrared spectra. Icarus, 189:35-62, July 2007. doi: 10.1016/j.icarus.2006.12.022.

Crida, A. and Charnoz, S. Formation of Regular Satellites from Ancient Massive Rings in the Solar System. Science, 338:1196, November 2012. doi: 10.1126/science.1226477.

Cumming, A., Marcy, G. W., and Butler, R. P. The Lick Planet Search: Detectability and Mass Thresholds. ApJ, 526:890-915, December 1999. doi: 10.1086/308020.

Cutri, R. M., Skrutskie, M. F., van Dyk, S., Beichman, C. A., Carpenter, J. M., Chester, T., Cambresy, L., Evans, T., Fowler, J., Gizis, J., Howard, E., Huchra, J., Jarrett, T., Kopan, E. L., Kirkpatrick, J. D., Light, R. M., Marsh, K. A., McCallon, H., Schneider, S., Stiening, R., Sykes, M., Weinberg, M., Wheaton, W. A., Wheelock, S., and Zacarias, N. VizieR Online Data Catalog: 2MASS All-Sky Catalog of Point Sources (Cutri+ 2003). VizieR Online Data Catalog, 2246, June 2003.

Deming, D., Wilkins, A., McCullough, P., Burrows, A., Fortney, J. J., Agol, E., DobbsDixon, I., Madhusudhan, N., Crouzet, N., Desert, J.-M., Gilliland, R. L., Haynes, K., Knutson, H. A., Line, M., Magic, Z., Mandell, A. M., Ranjan, S., Charbonneau, D., Clampin, M., Seager, S., and Showman, A. P. Infrared Transmission Spectroscopy of the Exoplanets HD 209458b and XO-1b Using the Wide Field Camera-3 on the Hubble Space Telescope. ApJ, 774:95, September 2013. doi: 10.1088/0004-637X/774/2/95.

Dobos, V., Heller, R., and Turner, E. L. The effect of multiple heat sources on exomoon habitable zones. AEFA, 601:A91, May 2017. doi: 10.1051/0004-6361/201730541.

Domingos, R. C., Winter, O. C., and Yokoyama, T. Stable satellites around extrasolar giant planets. MNRAS, 373:1227-1234, December 2006. doi: 10.1111/j.1365-2966. 2006.11104.x.

Dumusque, X., Pepe, F., Lovis, C., Ségransan, D., Sahlmann, J., Benz, W., Bouchy, F., Mayor, M., Queloz, D., Santos, N., and Udry, S. An Earth-mass planet orbiting $\alpha$ Centauri B. Nature, 491:207-211, November 2012. doi: 10.1038/nature11572.

Durbin, J. and Watson, G. S. Testing for serial correlation in least squares regression: I. Biometrika, 37(3/4):409-428, 1950. ISSN 00063444.

Foreman-Mackey, D., Hogg, D. W., Lang, D., and Goodman, J. emcee: The MCMC Hammer. PASP, 125:306, March 2013. doi: 10.1086/670067.

Forgan, D. H. On the feasibility of exomoon detection via exoplanet phase curve spectral contrast. MNRAS, 470(1):416-426, 2017. doi: 10.1093/mnras/stx1217. 
Gaia Collaboration, Prusti, T., de Bruijne, J. H. J., Brown, A. G. A., Vallenari, A., Babusiaux, C., Bailer-Jones, C. A. L., Bastian, U., Biermann, M., Evans, D. W., and et al. The Gaia mission. AEFA, 595:A1, November 2016. doi: 10.1051/0004-6361/201629272.

Gaia Collaboration, Brown, A. G. A., Vallenari, A., Prusti, T., de Bruijne, J. H. J., Babusiaux, C., Bailer-Jones, C. A. L., Biermann, M., Evans, D. W., Eyer, L., and et al. Gaia Data Release 2. Summary of the contents and survey properties. AEFA, 616:A1, August 2018. doi: 10.1051/0004-6361/201833051.

Gibson, N. P., Aigrain, S., Roberts, S., Evans, T. M., Osborne, M., and Pont, F. A Gaussian process framework for modelling instrumental systematics: application to transmission spectroscopy. MNRAS, 419:2683-2694, January 2012. doi: 10.1111/j.1365-2966. 2011.19915.x.

Gilliland, R. L., Chaplin, W. J., Dunham, E. W., Argabright, V. S., Borucki, W. J., Basri, G., Bryson, S. T., Buzasi, D. L., Caldwell, D. A., Elsworth, Y. P., Jenkins, J. M., Koch, D. G., Kolodziejczak, J., Miglio, A., van Cleve, J., Walkowicz, L. M., and Welsh, W. F. Kepler Mission Stellar and Instrument Noise Properties. ApJS, 197:6, November 2011. doi: 10.1088/0067-0049/197/1/6.

Gillon, M., Triaud, A. H. M. J., Demory, B.-O., Jehin, E., Agol, E., Deck, K. M., Lederer, S. M., de Wit, J., Burdanov, A., Ingalls, J. G., Bolmont, E., Leconte, J., Raymond, S. N., Selsis, F., Turbet, M., Barkaoui, K., Burgasser, A., Burleigh, M. R., Carey, S. J., Chaushev, A., Copperwheat, C. M., Delrez, L., Fernand es, C. S., Holdsworth, D. L., Kotze, E. J., Van Grootel, V., Almleaky, Y., Benkhaldoun, Z., Magain, P., and Queloz, D. Seven temperate terrestrial planets around the nearby ultracool dwarf star TRAPPIST-1. Nature, 542:456-460, Feb 2017. doi: 10.1038/nature21360.

Han, C. and Han, W. On the Feasibility of Detecting Satellites of Extrasolar Planets via Microlensing. ApJ, 580:490-493, November 2002. doi: 10.1086/343082.

Heller, R. Detecting Extrasolar Moons Akin to Solar System Satellites with an Orbital Sampling Effect. ApJ, 787:14, May 2014. doi: 10.1088/0004-637X/787/1/14.

Heller, R. The nature of the giant exomoon candidate Kepler-1625 b-i. AEFA, 610:A39, February 2018a. doi: 10.1051/0004-6361/201731760.

Heller, R. Detecting and Characterizing Exomoons and Exorings, page 35. Springer, 2018b. doi: 10.1007/978-3-319-55333-7_35.

Heller, R. and Albrecht, S. How to Determine an Exomoon's Sense of Orbital Motion. ApJ, 796:L1, November 2014. doi: 10.1088/2041-8205/796/1/L1.

Heller, R., Williams, D., Kipping, D., Limbach, M. A., Turner, E., Greenberg, R., Sasaki, T., Bolmont, É., Grasset, O., Lewis, K., Barnes, R., and Zuluaga, J. I. Formation, Habitability, and Detection of Extrasolar Moons. Astrobiology, 14:798-835, September 2014. doi: 10.1089/ast.2014.1147. 
Heller, R., Marleau, G. D., and Pudritz, R. E. The formation of the Galilean moons and Titan in the Grand Tack scenario. AEFA, 579:L4, July 2015. doi: 10.1051/0004-6361/ 201526348.

Heller, R., Hippke, M., and Jackson, B. Modeling the Orbital Sampling Effect of Extrasolar Moons. ApJ, 820:88, April 2016a. doi: 10.3847/0004-637X/820/2/88.

Heller, R., Hippke, M., Placek, B., Angerhausen, D., and Agol, E. Predictable patterns in planetary transit timing variations and transit duration variations due to exomoons. AEFA, 591:A67, June 2016b. doi: 10.1051/0004-6361/201628573.

Heller, R. Detecting and Characterizing Exomoons and Exorings, pages 1-17. Springer International Publishing, Cham, 2017. ISBN 978-3-319-30648-3. doi: 10.1007/ 978-3-319-30648-3_35-1.

Heller, R., Rodenbeck, K., and Bruno, G. An alternative interpretation of the exomoon candidate signal in the combined Kepler and Hubble data of Kepler-1625. AEFA, 624: A95, Apr 2019. doi: 10.1051/0004-6361/201834913.

Hippke, M. On the Detection of Exomoons: A Search in Kepler Data for the Orbital Sampling Effect and the Scatter Peak. ApJ, 806:51, June 2015. doi: 10.1088/0004-637X/ $806 / 1 / 51$.

Hippke, M. Synthetic dataset for the exomoon candidate around kepler-1625 b, 2018.

Hippke, M. and Heller, R. Optimized transit detection algorithm to search for periodic transits of small planets. AEAA, 623:A39, Mar 2019. doi: 10.1051/0004-6361/ 201834672.

Horne, K. An optimal extraction algorithm for CCD spectroscopy. PASP, 98:609-617, June 1986. doi: 10.1086/131801.

Howard, A. W. Observed Properties of Extrasolar Planets. Science, 340:572-576, May 2013. doi: $10.1126 /$ science. 1233545 .

Kass, R. E. and Raftery, A. E. Bayes factors. Journal of the American Statistical Association, 90(430):773-795, 1995. doi: 10.1080/01621459.1995.10476572.

Kipping, D. M. Transit timing effects due to an exomoon. MNRAS, 392:181-189, January 2009. doi: 10.1111/j.1365-2966.2008.13999.x.

Kipping, D. M. LUNA: an algorithm for generating dynamic planet-moon transits. $M N$ $R A S, 416: 689-709$, September 2011. doi: 10.1111/j.1365-2966.2011.19086.x.

Kipping, D. M. Efficient, uninformative sampling of limb darkening coefficients for two-parameter laws. MNRAS, 435:2152-2160, November 2013. doi: 10.1093/mnras/ stt1435.

Kipping, D. M., Bakos, G. Á., Buchhave, L., Nesvorný, D., and Schmitt, A. The Hunt for Exomoons with Kepler (HEK). I. Description of a New Observational project. ApJ, 750:115, May 2012. doi: 10.1088/0004-637X/750/2/115. 
Kipping, D. M., Forgan, D., Hartman, J., Nesvorný, D., Bakos, G. Á., Schmitt, A., and Buchhave, L. The Hunt for Exomoons with Kepler (HEK). III. The First Search for an Exomoon around a Habitable-zone Planet. ApJ, 777:134, November 2013a. doi: 10.1088/0004-637X/777/2/134.

Kipping, D. M., Hartman, J., Buchhave, L. A., Schmitt, A. R., Bakos, G. Á., and Nesvorný, D. The Hunt for Exomoons with Kepler (HEK). II. Analysis of Seven Viable Satellite-hosting Planet Candidates. ApJ, 770:101, June 2013b. doi: 10.1088/ 0004-637X/770/2/101.

Kipping, D. M., Nesvorný, D., Buchhave, L. A., Hartman, J., Bakos, G. Á., and Schmitt, A. R. The Hunt for Exomoons with Kepler (HEK). IV. A Search for Moons around Eight M Dwarfs. ApJ, 784:28, March 2014. doi: 10.1088/0004-637X/784/1/28.

Kipping, D. M., Schmitt, A. R., Huang, X., Torres, G., Nesvorný, D., Buchhave, L. A., Hartman, J., and Bakos, G. Á. The Hunt for Exomoons with Kepler (HEK): V. A Survey of 41 Planetary Candidates for Exomoons. ApJ, 813:14, November 2015. doi: 10.1088/0004-637X/813/1/14.

Kipping, D. M. How to weigh a star using a moon. MNRAS, 409:L119-L123, November 2010. doi: 10.1111/j.1745-3933.2010.00961.x.

Koch, D. G., Borucki, W. J., Basri, G., Batalha, N. M., Brown, T. M., Caldwell, D., Christensen-Dalsgaard, J., Cochran, W. D., DeVore, E., Dunham, E. W., Gautier, I., Thomas N., Geary, J. C., Gilliland, R. L., Gould, A., Jenkins, J., Kondo, Y., Latham, D. W., Lissauer, J. J., Marcy, G., Monet, D., Sasselov, D., Boss, A., Brownlee, D., Caldwell, J., Dupree, A. K., Howell, S. B., Kjeldsen, H., Meibom, S., Morrison, D., Owen, T., Reitsema, H., Tarter, J., Bryson, S. T., Dotson, J. L., Gazis, P., Haas, M. R., Kolodziejczak, J., Rowe, J. F., Van Cleve, J. E., Allen, C., Chandrasekaran, H., Clarke, B. D., Li, J., Quintana, E. V., Tenenbaum, P., Twicken, J. D., and $\mathrm{Wu}, \mathrm{H}$. Kepler Mission Design, Realized Photometric Performance, and Early Science. ApJ, 713:L79-L86, April 2010. doi: 10.1088/2041-8205/713/2/L79.

Krizhevsky, A., Sutskever, I., and Hinton, G. E. Imagenet Classification with Deep Convolutional Neural Networks. Advances in Neural Information Processing Systems 25, pages 1097-1105, 2012.

Laskar, J., Joutel, F., and Robutel, P. Stabilization of the earth's obliquity by the moon. Nature, 361:615-617, February 1993. doi: 10.1038/361615a0.

Lecavelier des Etangs, A., Hébrard, G., Blandin, S., Cassier, J., Deeg, H. J., Bonomo, A. S., Bouchy, F., Désert, J.-M., Ehrenreich, D., Deleuil, M., Díaz, R. F., Moutou, C., and Vidal-Madjar, A. Search for rings and satellites around the exoplanet CoRoT-9b using Spitzer photometry. AEFA, 603:A115, July 2017. doi: 10.1051/0004-6361/201730554.

Lewis, K. M. and Fujii, Y. Next Generation of Telescopes or Dynamics Required to Determine if Exo-moons have Prograde or Retrograde Orbits. ApJ, 791:L26, August 2014. doi: 10.1088/2041-8205/791/2/L26. 
Liu, S. and Deng, W. Very Deep Convolutional Neural Network Based Image Classification Using Small Training Sample Size. In $3^{\text {rd }}$ IAPR Asian Conference on Pattern Recognition, pages 730-734, 2015.

Mandel, K. and Agol, E. Analytic Light Curves for Planetary Transit Searches. ApJ, 580: L171-L175, December 2002. doi: 10.1086/345520.

Martin, D. V., Fabrycky, D. C., and Montet, B. T. Transits of Inclined Exomoons - Hide and Seek and an Application to Kepler-1625. arXiv e-prints, January 2019.

Mathur, S., Huber, D., Batalha, N. M., Ciardi, D. R., Bastien, F. A., Bieryla, A., Buchhave, L. A., Cochran, W. D., Endl, M., Esquerdo, G. A., Furlan, E., Howard, A., Howell, S. B., Isaacson, H., Latham, D. W., MacQueen, P. J., and Silva, D. R. Revised Stellar Properties of Kepler Targets for the Q1-17 (DR25) Transit Detection Run. ApJS, 229: 30, April 2017. doi: 10.3847/1538-4365/229/2/30.

Mayor, M. and Queloz, D. A Jupiter-mass companion to a solar-type star. Nature, 378: 355-359, November 1995. doi: 10.1038/378355a0.

Mayor, M., Marmier, M., Lovis, C., Udry, S., Ségransan, D., Pepe, F., Benz, W., Bertaux, J. L., Bouchy, F., Dumusque, X., Lo Curto, G., Mordasini, C., Queloz, D., and Santos, N. C. The HARPS search for southern extra-solar planets XXXIV. Occurrence, mass distribution and orbital properties of super-Earths and Neptune-mass planets. arXiv e-prints, art. arXiv:1109.2497, September 2011.

McCullough, P. and MacKenty, J. Considerations for using Spatial Scans with WFC3. Technical report, May 2012.

Moskovitz, N. A., Gaidos, E., and Williams, D. M. The Effect of Lunarlike Satellites on the Orbital Infrared Light Curves of Earth-Analog Planets. Astrobiology, 9:269-277, April 2009. doi: 10.1089/ast.2007.0209.

Namouni, F. The Fate of Moons of Close-in Giant Exoplanets. ApJ, 719:L145-L147, August 2010. doi: 10.1088/2041-8205/719/2/L145.

Peters, M. A. and Turner, E. L. On the Direct Imaging of Tidally Heated Exomoons. ApJ, 769:98, Jun 2013. doi: 10.1088/0004-637X/769/2/98.

Petigura, E. A., Howard, A. W., and Marcy, G. W. Prevalence of Earth-size planets orbiting Sun-like stars. Proceedings of the National Academy of Science, 110:1927319278, November 2013. doi: 10.1073/pnas.1319909110.

Pirzkal, N., Ryan, R., and Brammer, G. Trace and Wavelength Calibrations of the WFC3 G102 and G141 IR Grisms. Technical report, September 2016.

Rajpaul, V., Aigrain, S., and Roberts, S. Ghost in the time series: no planet for Alpha Cen B. MNRAS, 456:L6-L10, February 2016. doi: 10.1093/mnrasl/slv164. 
Rauer, H., Catala, C., Aerts, C., Appourchaux, T., Benz, W., Brandeker, A., ChristensenDalsgaard, J., Deleuil, M., Gizon, L., Goupil, M.-J., Güdel, M., Janot-Pacheco, E., Mas-Hesse, M., Pagano, I., Piotto, G., Pollacco, D., Santos, C., Smith, A., Suárez, J.C., Szabó, R., Udry, S., Adibekyan, V., Alibert, Y., Almenara, J.-M., Amaro-Seoane, P., Eiff, M. A.-v., Asplund, M., Antonello, E., Barnes, S., Baudin, F., Belkacem, K., Bergemann, M., Bihain, G., Birch, A. C., Bonfils, X., Boisse, I., Bonomo, A. S., Borsa, F., Brandão, I. M., Brocato, E., Brun, S., Burleigh, M., Burston, R., Cabrera, J., Cassisi, S., Chaplin, W., Charpinet, S., Chiappini, C., Church, R. P., Csizmadia, S., Cunha, M., Damasso, M., Davies, M. B., Deeg, H. J., Díaz, R. F., Dreizler, S., Dreyer, C., Eggenberger, P., Ehrenreich, D., Eigmüller, P., Erikson, A., Farmer, R., Feltzing, S., de Oliveira Fialho, F., Figueira, P., Forveille, T., Fridlund, M., García, R. A., Giommi, P., Giuffrida, G., Godolt, M., Gomes da Silva, J., Granzer, T., Grenfell, J. L., Grotsch-Noels, A., Günther, E., Haswell, C. A., Hatzes, A. P., Hébrard, G., Hekker, S., Helled, R., Heng, K., Jenkins, J. M., Johansen, A., Khodachenko, M. L., Kislyakova, K. G., Kley, W., Kolb, U., Krivova, N., Kupka, F., Lammer, H., Lanza, A. F., Lebreton, Y., Magrin, D., Marcos-Arenal, P., Marrese, P. M., Marques, J. P., Martins, J., Mathis, S., Mathur, S., Messina, S., Miglio, A., Montalban, J., Montalto, M., Monteiro, M. J. P. F. G., Moradi, H., Moravveji, E., Mordasini, C., Morel, T., Mortier, A., Nascimbeni, V., Nelson, R. P., Nielsen, M. B., Noack, L., Norton, A. J., Ofir, A., Oshagh, M., Ouazzani, R.-M., Pápics, P., Parro, V. C., Petit, P., Plez, B., Poretti, E., Quirrenbach, A., Ragazzoni, R., Raimondo, G., Rainer, M., Reese, D. R., Redmer, R., Reffert, S., Rojas-Ayala, B., Roxburgh, I. W., Salmon, S., Santerne, A., Schneider, J., Schou, J., Schuh, S., Schunker, H., Silva-Valio, A., Silvotti, R., Skillen, I., Snellen, I., Sohl, F., Sousa, S. G., Sozzetti, A., Stello, D., Strassmeier, K. G., Švanda, M., Szabó, G. M., Tkachenko, A., Valencia, D., Van Grootel, V., Vauclair, S. D., Ventura, P., Wagner, F. W., Walton, N. A., Weingrill, J., Werner, S. C., Wheatley, P. J., and Zwintz, K. The PLATO 2.0 mission. Experimental Astronomy, 38: 249-330, November 2014. doi: 10.1007/s10686-014-9383-4.

Reynolds, R. T., McKay, C. P., and Kasting, J. F. Europa, tidally heated oceans, and habitable zones around giant planets. Advances in Space Research, 7:125-132, 1987. doi: 10.1016/0273-1177(87)90364-4.

Ricker, G. R., Winn, J. N., Vanderspek, R., Latham, D. W., Bakos, G. Á., Bean, J. L., Berta-Thompson, Z. K., Brown, T. M., Buchhave, L., Butler, N. R., Butler, R. P., Chaplin, W. J., Charbonneau, D., Christensen-Dalsgaard, J., Clampin, M., Deming, D., Doty, J., De Lee, N., Dressing, C., Dunham, E. W., Endl, M., Fressin, F., Ge, J., Henning, T., Holman, M. J., Howard, A. W., Ida, S., Jenkins, J. M., Jernigan, G., Johnson, J. A., Kaltenegger, L., Kawai, N., Kjeldsen, H., Laughlin, G., Levine, A. M., Lin, D., Lissauer, J. J., MacQueen, P., Marcy, G., McCullough, P. R., Morton, T. D., Narita, N., Paegert, M., Palle, E., Pepe, F., Pepper, J., Quirrenbach, A., Rinehart, S. A., Sasselov, D., Sato, B., Seager, S., Sozzetti, A., Stassun, K. G., Sullivan, P., Szentgyorgyi, A., Torres, G., Udry, S., and Villasenor, J. Transiting Exoplanet Survey Satellite (TESS). Journal of Astronomical Telescopes, Instruments, and Systems, 1(1):014003, January 2015. doi: 10.1117/1.JATIS.1.1.014003. 
Rodenbeck, K., Heller, R., Hippke, M., and Gizon, L. Revisiting the exomoon candidate signal around Kepler-1625 b. AEFA, 617:A49, September 2018. doi: 10.1051/ 0004-6361/201833085.

Sartoretti, P. and Schneider, J. On the detection of satellites of extrasolar planets with the method of transits. AEFAS, 134:553-560, February 1999. doi: 10.1051/aas:1999148.

Schwarz, G. Estimating the Dimension of a Model. Annals of Statistics, 6:461-464, July 1978.

Seager, S. and Mallén-Ornelas, G. A Unique Solution of Planet and Star Parameters from an Extrasolar Planet Transit Light Curve. ApJ, 585:1038-1055, March 2003. doi: $10.1086 / 346105$.

Simon, A., Szatmáry, K., and Szabó, G. Determination of the size, mass, and density of "exomoons" from photometric transit timing variations. AEFA, 470:727-731, August 2007. doi: 10.1051/0004-6361:20066560.

Simon, A., Szabó, G., Kiss, L., and Szatmáry, K. Signals of exomoons in averaged light curves of exoplanets. MNRAS, 419:164-171, January 2012. doi: 10.1111/j.1365-2966. 2011.19682.x.

Simon, A. E., Szabó, G. M., Szatmáry, K., and Kiss, L. L. Methods for exomoon characterization: combining transit photometry and the Rossiter-McLaughlin effect. MNRAS, 406:2038-2046, August 2010. doi: 10.1111/j.1365-2966.2010.16818.x.

Simon, A. E., Szabó, G. M., Kiss, L. L., Fortier, A., and Benz, W. CHEOPS Performance for Exomoons: The Detectability of Exomoons by Using Optimal Decision Algorithm. Publications of the Astronomical Society of the Pacific, 127:1084, October 2015. doi: $10.1086 / 683392$.

Spiegelhalter, D. J., Best, N. G., Carlin, B. P., and Van Der Linde, A. Bayesian measures of model complexity and fit. Journal of the Royal Statistical Society: Series B (Statistical Methodology), 64(4):583-639, 2002. doi: 10.1111/1467-9868.00353.

Spohn, T. and Schubert, G. Oceans in the icy Galilean satellites of Jupiter? Icarus, 161: 456-467, February 2003. doi: 10.1016/S0019-1035(02)00048-9.

Stevenson, K. B., Harrington, J., Fortney, J. J., Loredo, T. J., Hardy, R. A., Nymeyer, S., Bowman, W. C., Cubillos, P., Bowman, M. O., and Hardin, M. Transit and Eclipse Analyses of the Exoplanet HD 149026b Using BLISS Mapping. ApJ, 754:136, August 2012. doi: 10.1088/0004-637X/754/2/136.

Szabó, G. M., Szatmáry, K., Divéki, Z., and Simon, A. Possibility of a photometric detection of "exomoons". AEFA, 450:395-398, April 2006. doi: 10.1051/0004-6361: 20054555.

Szabó, R., Szabó, G., Dálya, G., Simon, A., Hodosán, G., and Kiss, L. Multiple planets or exomoons in Kepler hot Jupiter systems with transit timing variations? AEFA, 553: A17, May 2013. doi: 10.1051/0004-6361/201220132. 
Teachey, A. and Kipping, D. M. Evidence for a large exomoon orbiting Kepler-1625b. Science Advances, 4:eaav1784, October 2018. doi: 10.1126/sciadv.aav1784.

Teachey, A., Kipping, D. M., and Schmitt, A. R. HEK. VI. On the Dearth of Galilean Analogs in Kepler, and the Exomoon Candidate Kepler-1625b I. AJ, 155:36, January 2018. doi: 10.3847/1538-3881/aa93f2.

Twicken, J. D., Jenkins, J. M., Seader, S. E., Tenenbaum, P., Smith, J. C., Brownston, L. S., Burke, C. J., Catanzarite, J. H., Clarke, B. D., Cote, M. T., Girouard, F. R., Klaus, T. C., Li, J., McCauliff, S. D., Morris, R. L., Wohler, B., Campbell, J. R., Kamal Uddin, A., Zamudio, K. A., Sabale, A., Bryson, S. T., Caldwell, D. A., Christiansen, J. L., Coughlin, J. L., Haas, M. R., Henze, C. E., Sanderfer, D. T., and Thompson, S. E. Detection of Potential Transit Signals in 17 Quarters of Kepler Data: Results of the Final Kepler Mission Transiting Planet Search (DR25). AJ, 152:158, December 2016. doi: 10.3847/0004-6256/152/6/158.

Vanderburg, A., Rappaport, S. A., and Mayo, A. W. Detecting Exomoons via Doppler Monitoring of Directly Imaged Exoplanets. AJ, 156:184, November 2018a. doi: 10. 3847/1538-3881/aae0fc.

Vanderburg, A., Rappaport, S. A., and Mayo, A. W. Detecting Exomoons Via Doppler Monitoring of Directly Imaged Exoplanets. ArXiv e-prints, May 2018b.

Wilkins, A. N., Deming, D., Madhusudhan, N., Burrows, A., Knutson, H., McCullough, P., and Ranjan, S. The Emergent 1.1-1.7 $\mu \mathrm{m}$ Spectrum of the Exoplanet CoRoT$2 \mathrm{~b}$ as Measured Using the Hubble Space Telescope. ApJ, 783:113, March 2014. doi: 10.1088/0004-637X/783/2/113.

Williams, D. M. Capture of Terrestrial-Sized Moons by Gas Giant Planets. Astrobiology, 13:315-323, April 2013. doi: 10.1089/ast.2012.0892.

Williams, D. M., Kasting, J. F., and Wade, R. A. Habitable moons around extrasolar giant planets. Nature, 385:234-236, January 1997. doi: 10.1038/385234a0.

Zhuang, Q., Gao, X., and Yu, Q. The Rossiter-McLaughlin Effect for Exomoons or Binary Planets. ApJ, 758:111, Oct 2012. doi: 10.1088/0004-637X/758/2/111.

Zollinger, R. R., Armstrong, J. C., and Heller, R. Exomoon habitability and tidal evolution in low-mass star systems. MNRAS, 472:8-25, November 2017. doi: 10.1093/mnras/ stx1861. 


\section{Publications}

\section{Refereed publications}

- Revisiting the exomoon candidate signal around Kepler-1625 b: Rodenbeck, K., Heller, R, Hippke, M., Gizon, L., 2018, A\&A, 617, A49

- An alternative interpretation of the exomoon candidate signal in the combined Kepler and Hubble data of Kepler-1625: Heller, R., Rodenbeck, K., Bruno, G. 2019, A\&A, 624, A95

- Sectoral $\mathrm{r}$ modes and periodic RV variations of Sun-like stars: Lanza, A. F., Gizon, L., Zaqarashvili, T. V., Liang, Z.-C., Rodenbeck, K., 2019, A\&A, 623, A50

\section{Submitted publications}

- The Transit Least Squares Survey I. Discovery and validation of an Earth-sized planet in the four-planet system K2-32 near the 1:2:5:7 resonance: Heller, R., Rodenbeck, K., Hippke, M., submitted to A\&A

\section{Conferences attended}

- Kepler Science Conference V, Glendale, CA, USA, 4.3.-8.3.2019, oral presentation: Revisiting the exomoon candidate signal around Kepler-1625 b

- Annual Meeting of the German Astronomical Society, 18.9.-22.9.2017, Göttingen, Germany

- Asteroseismology and Exoplanets: Listening to the Stars and Searching for New Worlds, Summer School, 17.7.-27.7.2016, Horta, Portugal 



\section{Acknowledgements}

This thesis would not have been possible without the help of many people who helped to guide and support me during my years as a PhD student.

First and foremost I'd like to thank my supervisor Laurent Gizon for all his advice and unwaivering support, even with the many jobs - managing director, professor, advisor to many $\mathrm{PhD}$ students - he has to handle. A heartfelt "thank you" also goes to the other members of my TAC: Stefan Dreizler, Matthias Ammler-von Eiff and Warrick Ball.

I'd like to thank Laurent Gizon, Stefan Dreizler, Ulrich Christensen, Saskia Hekker, René Heller and Wolfram Kollatschny for being part of my thesis defense committee.

I'd also like to thank René Heller for all the time and effort he expended in helping me becoming a better scientist. Even though he only arrived halfway through my $\mathrm{PhD}$, he was a great mentor to me. I enjoyed the collaborations with Michael Hippke, Giovanni Bruno, and Rasha Alshehhi.

All the people in the Solar and Stellar Interiors Department always had their doors open to listen to my questions, to help where they could, or just to have a nice chat, for all of which I'm very grateful. Especially I'd like to thank Jesper Schou, who is always open for discussions about everything: astrophysics, statistics, general science, language, vaccinations, traffic laws or just office gossip. The list of topics really is endless.

The students of the IMPRS have managed to form a great community at the institute. They make every new student feel welcome, are always supportive and (almost fanatically) inclusive.

Lastly I'd like to thank Sonja Schuh for helping me navigate the IMPRS and university regulations and in general for working tirelessly to smooth the students' path towards graduation.

Natürlich möchte ich auch meiner Familie für ihre endlose Unterstützung danken. Vor allem meine Mutter war eine große Hilfe, sei es in Rat oder Tat. 



\section{Curriculum vitae}

\section{Personal Details}

Date of Birth

August 6, 1989

Place of Birth

Nationality

Göttingen, Germany

German

\section{Education}

\section{PhD student}

$4 / 2015-4 / 2019$

at the International Max Planck Research School for Solar System Science and the University of Göttingen

Thesis On the Detection of Exomoons in Photometric Time Series

Advisor Prof. Dr. Gizon

Thesis Advisory Prof. Dr. Gizon, Prof. Dr. Dreizler, Dr. Warrick Ball

Committee

\section{M.Sc. in Physics}

9/2012-1/2015

at University of Göttingen

Focus

Astro- and Geophysics

Thesis

Magnetic Field Amplification in Galaxy Mergers

Advisor

Prof. Dr. Schleicher

\section{B.Sc. in Physics}

$$
\begin{aligned}
\text { 9/2009 - 8/2012 } & \text { at University of Göttingen } \\
\text { Thesis } & \text { Magnetic Fields in Primordial Gas Disks } \\
\text { Advisor } & \text { Prof. Dr. Schleicher }
\end{aligned}
$$

\section{School}

8/2002 - 7/2009 Max-Planck-Gymnasium, Göttingen, concluded with Abitur 